\title{
SOBRE UM MODELO LINEAR PARA ENSAIOS EM "SWITCHBACK" DELINEADOS EM BLOCOS CASUALIZADOS
}

\author{
MARIA ANGELICA FERRAZ DE TOLEDO MACHADO
}

Orientador: Prof. Dr. ANTONIO FRANCISCO IEMMA

Dissertação apresentada à Escola

Superior de Agricultura "Luiz de Queiroz", da Universidade de São Paulo, para obtenção do título de Mestre em Agronomia. Área de Concentração: Estatística e Expo. rimentação Agronômica.

\author{
PIRACICABA \\ Estado de São Paulo - Brasil \\ Maio - 1984
}


i i .

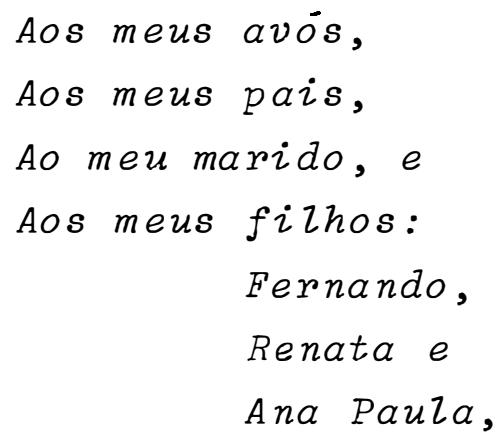


i i i.

\section{AGRADEC IMENTOS}

Ao Dr. Antonio Francisco Iemma, Professor Assistente Dou tor do Departamento de Matemática e Estatística da ESALQ, pela orientação na execução deste trabalho.

Ao Dr. Décio Barbin, Professor Titular do Departamento de Matemática e Estatística da ESALQ, pela orientação e apoio concedido no decorrer do Curso.

Ao Dr. Humberto de Campos, Professor Titular do Departamento de Matemática e Estatística da ESALQ, pelo apoio concedido no decorrer do Curso.

Ao Dr. Cássio Roberto de Melo Godoi, Professor Livre-Docente do Departamento de Matemática e Estatística da ESALQ, pelos ensinamentos.

Aos Professores do Curso de Pós-Graduação em Estatística e Experimentação Agronômica da ESALQ, pelos ensinamen tos.

A Diretoria do Instituto de Economia Agrícola da Secretaria da Agricultura, pelo afastamento concedido.

Ao meu pai, Dr. Francisco Ferraz de Toledo, Professor Ti tular do Departamento de Agricultura e Horticultura da ESALQ, pelo apoio, incentivo e revisão final do traba 1ho. 
A Srta. Maria Izalina Ferreira Alves, pela datilografia deste trabalho.

Aos colegas de Pós-Graduação pela convivência.

Aos Professores Dr. Raul Dantas D'Arce, Dr. Wilson Mattos e Dr. Paulo Machado, pelo fornecimento dos dados.

Aos Funcionários do Departamento de Matemática e Estatística da ESALQ, pela colaboração.

A todos que direta ou indiretamente colaboraram para a realização deste trabalho. 
Pág.

RESUMO $\ldots \ldots \ldots \ldots \ldots \ldots \ldots \ldots \ldots \ldots \ldots \ldots \ldots \ldots \ldots \ldots \ldots \ldots \ldots$

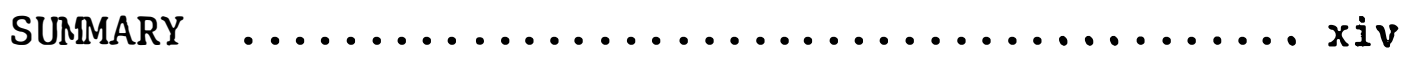

1. INTRODUÇAO $\ldots \ldots \ldots \ldots \ldots \ldots \ldots \ldots \ldots \ldots \ldots \ldots$

2. REVISÃo BIBLIOGRAFICA $\ldots \ldots \ldots \ldots \ldots \ldots \ldots \ldots \ldots$

2.1 - Generalidades $\ldots \ldots \ldots \ldots \ldots \ldots \ldots \ldots \ldots$

2.2 - Modelo Matemático $\ldots \ldots \ldots \ldots \ldots \ldots \ldots \ldots$

2.3 - Sistema de Equa ções Normais ......... 9

2.4 - Solução do Sistema dé Equações Normais, Nnālise de Variância e Comparações Múltiplas ............ 11

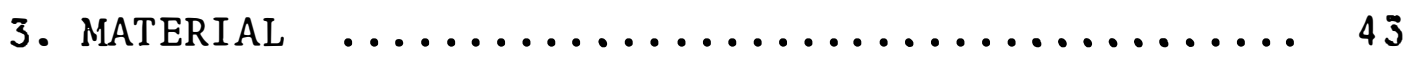

4. Desenvolvimento térico $\ldots \ldots \ldots \ldots \ldots \ldots \ldots \ldots .46$

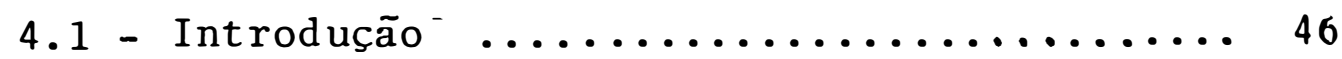

4.2 - Adoção de Um Modelo Linear .......... 48

4.2 .1 - Exigências do modelo adotado .... 49

4.3 - Equações Normais $\ldots \ldots \ldots \ldots \ldots \ldots \ldots \ldots$

4.3 .1 - Solução do sistema para a média .. 64

4.3.2 - Solução do sistema para efeitos de tratamentos eliminando-se efeitos de vacas $\ldots \ldots \ldots \ldots \ldots \ldots \ldots \ldots 65$

4.3 .3 - Solução do sistema para vacas ... 70

4.3.4 - Solução do sistema para efeitas lí near e quadrático de períodos ... 71 
v1.

Pî̀g.

4.3.5 - Solução do sistema para efeitos de

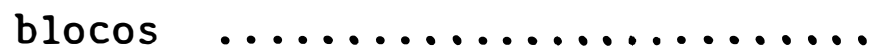

4.3 .6 - Solução do sistema para efeitos da interação período (linear) x vacas 73

4.4 - Somas de Quadrados ................. 74

4.4.1 - Soma de quadrados devida à média.. 75

4.4.2 - Soma de quadrados devida aos efeitos de blocos ............... 75

4.4.3 - Soma de quadrados devida aos efeitos de vacas ignorando efeitos de tratamentos

4.4.4 - Soma de quadrados devida a efeitos de tratamentos .............. 78

4.4.5 - Soma de quadrados devida a efeitos de períodos ................ 81

4.4.6 - Soma de quadrados devida a interação período (linear) x vacas .... 82

$\overline{4} .5$ - Esperanças Matemāticas .............. 83

4.5 .1 - Esperança Matemätica de $\mathrm{B}^{\prime} \mathrm{M}_{7}^{-1} \mathrm{~B} \ldots 83$

4.5 .2 - Esperança Matemātica de $V^{\prime} M_{12}^{-1} \mathrm{~V}$.. 85

4.5 .3 - Esperança Matemātica de $\bar{\tau}^{\prime} Q^{*} \ldots$... 86

4.5 .4 - Esperança Matemätica de $\mathrm{P}^{\prime} \mathrm{M}_{39}^{-1} \mathrm{p} \ldots$ SS

4.5 .5 - Esperança Matemātica de $\mathrm{C}^{\prime} \mathrm{M}_{23}^{-1} \mathrm{C} \ldots 89$

4.5 .6 - Esperança Matemätica de y'y .... 9) 
v1j.

päg.

4.5.7 - Esperança Matemātica da correção $M_{1}^{-1}\left[y^{\prime} x_{1} x_{1}^{\prime} y\right] \quad \ldots \ldots \ldots \ldots \ldots \ldots$

4.5.8 - Esperança Matemática da correção para blocos ...............

4.5.9 - Esperança Matemātica de

$$
\frac{1}{v \sum x_{j}^{2}}\left(P_{3}-P_{1}\right)^{2} \ldots \ldots
$$

4.5.10 - Esperança Matemática de

$$
\frac{1}{\Sigma x_{j}^{2}}\left[y^{\prime} x_{6} x_{6}^{\prime} y\right] \quad \ldots \ldots \ldots
$$

96

4.5.11 - Esperança Matemātica da soma de quadrados devida a efeito de blo $\cos \ldots \ldots \ldots \ldots \ldots \ldots \ldots \ldots$

4.5.12 - Esperança Matemática da soma de quadrados devida a efeitos de va cas ignorando tratamentos .....

4.5.13 - Esperança Matemātica da soma de quadrados devida a efeitos de tra tamentos (ajustada) ............

4.5.14 - Esperança Matemática da soma de quadrados devida a efeitos de pe

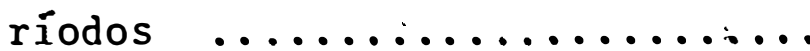

4.5.15 - Esperança Matemática da soma de quadrados devida a efeitos da in teração período (linear) $x$ vacas 
vij.

Pảg.

4.5 .16 - Esperança Matemātica da soma de quadrado do resíduo $\ldots \ldots \ldots \ldots$ 101

4.6 - Distribuição das Formas Quadrāticas ... 104 4.6 .1 - Distribuição de $B^{1} M_{7}^{-1} B^{\prime} \sigma^{2} \ldots \ldots l 105$

4.6 .2 - Distribuição de $V^{\prime} M_{12}^{-1} V_{/} / \sigma^{2} \ldots \ldots 100$

4.6 .3 - Distribuição de $\hat{\tau}^{\prime} Q^{*} / \sigma^{2} \ldots \ldots \ldots .108$

4.6 .4 - Distribuição de $\mathrm{P}^{\prime} \mathrm{M}_{19}^{-1} \mathrm{P} / \sigma^{2} \ldots \ldots \quad 110$

4.6 .5 - Distribuição de $C^{\prime} M_{21}^{-1} C_{/} \sigma^{2} \quad \ldots \ldots 111$

4.6 .6 - Distribuição de $y^{\prime} y / \sigma^{2} \ldots \ldots \ldots 112$

4.6 .7 - Distribuição da $\mathrm{SQR} / \sigma^{2} \ldots \ldots \ldots 113$

" 4.7 - Distribuição dos Quocientes ........ 115

4.7.1 - Distribuição do quociente

$$
\frac{\mathrm{B}^{\prime} \mathrm{M}_{7}^{-1} \mathrm{~B} / \mathrm{n}_{I} \cdot \sigma^{2}}{\mathrm{~S} Q \mathrm{R} / \mathrm{n}_{7} \cdot \sigma^{2}}
$$

4.7.2 - Distribuição do quociente

$$
\frac{V^{\prime} M_{12}^{-1} V / n_{2} \cdot \sigma^{2}}{S Q R / n_{7} \cdot \sigma^{2}}
$$

4.7.3 - Distribuição do quociente

$$
\frac{\hat{\tau}^{\prime} Q^{*} / n_{3} \cdot \sigma^{2}}{\mathrm{SQR} / \mathrm{n}_{7} \cdot \sigma^{2}}
$$

4.7.4 - Distribuição do quociente

$$
\frac{P^{\prime} M_{19}^{-1} P / n_{4} \cdot \sigma^{2}}{S Q R / n_{7} \cdot \sigma^{2}}
$$


4.7.5 - Distribuição dò quociente

$$
\frac{C^{\prime} M_{21}^{-1} C / n_{5} \cdot \sigma^{2}}{S Q R / n_{7} \cdot \sigma^{2}}
$$

4.8 - Testes de Hipóteses e Quadro da Análise de

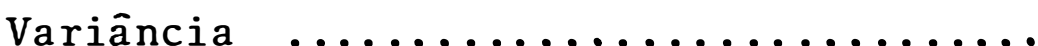

4.9 - Comparações Múltiplas ............ 122

5. ILUSTRAÇAO DO METODO PROPOSTO $\ldots \ldots \ldots \ldots \ldots \ldots .123$

5.1 - Modelo Matemático ................. 123

5.2 - Equações Normais .............. 128

5.3 - Solução do Sistema de Equações Normais .. 133

5.3 .1 - Estimativa da média $\ldots \ldots \ldots \ldots .133$

5.3.2 - Estimativa dos efeitos de tratamen tos eliminando-se efeitos de vacas 153

5.3 .3 - Estimativa dos efeitos de vacas .. 1.35

5.3.4 - Estimativa dos efeitos linear e qui drático de períodos .......... 1.30

5.3.5 - Estimativa dos efeitos de blocos.. 157

5.3.6 - Estimativa dos efeitos da interação período (linear) x vacas ... 157

5.4 - Somas de Quadrados .............. Jis

5.4 .1 - Obtenção da soma de quadrados de iî 1 is

5.4 .2 - Obtenção da soma de quadrados de blocos ...................... Is 
5.4.3 - Obtenção da soma de quadrados de tratamentos (ajustada) $\ldots \ldots \ldots$ 13!)

5.4.4 - Obtenção da soma de quadrados de vacas ................. 139

5.4.5 - Obtenção da soma de quadrados de períodos ............... J.3?

5.46 - Obtenção da soma de quadrados da interação períolo (linear) $x$ vacas $\ldots \ldots \ldots \ldots \ldots \ldots \ldots \ldots \ldots$. 140

5.5 - Quadro da Anālise de Varianncia e Testes de Hipóteses ..................... 1, 10

5.6 - Compa rações Mültiplas $\ldots \ldots \ldots \ldots \ldots \ldots$ 1

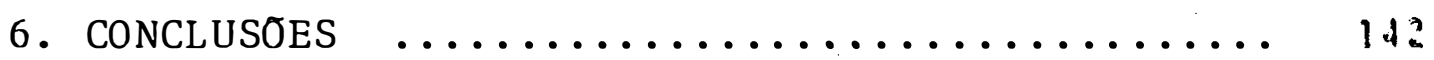

7. REFERENCIAS BIBLIOGRAFICAS $\ldots \ldots \ldots \ldots \ldots \ldots . \ldots \ldots$ 


\section{SOBRE UM MODELO LINEAR PARA ENSAIOS EM "SWITCHBACK"} DELINEADOS EM BLOCOS CASUALIZADOS

Autor: Maria Angélica Ferraz de Toledo Machado Orientador: Dr. Antonio Francisco I emma

Com este trabalho, pretendeu-se estabelecer um modelo linear para ensaios em "switchback", ronde o efeito de blocos estivesse presente.

0 modelo adotado foi o seguinte:

$$
\begin{aligned}
y_{i j k \ell}=m & +b_{\ell} z_{j}+v_{i}+t_{k}+\alpha_{1} x_{j}+\alpha_{2} z_{j}+c_{i} x_{j}+ \\
& +e_{i j k \ell}
\end{aligned}
$$

onde, para $i=1,2, \ldots, v ; j=1,2, \ldots, J ; k=1,2, \ldots, p ; l=1,2$, $\ldots, \mathrm{L}$,

$y_{i j k \ell}$ é o valor observado na i-ésima vaca, do j-êsimo período, submetida ao k-ésimo tratamento do $\mathrm{p}-$ -ésimo bloco;

m é a média geral; 
$\mathrm{b}_{\ell}$ é o efeito do bloco $\ell$;

$v_{i}$ é o efeito da vaca $\underline{i}$;

$t_{k}$ é o efeito do tratamento $\underline{k}$;

$\alpha_{1}$ è o efeito linear de período;

$\alpha_{2}$ é o efeito quadrātico de período;

$c_{i}$ é o efeito da interação período (linear) $x$ vacas; $e_{i j k \ell}$ é o erro experimental onde se supõe que

$$
e_{i j k \ell} \cap N\left(0, \sigma^{2}\right)
$$

e que todos os erros experimentais sejam independentes;

e

$$
\begin{aligned}
& x_{j}=\left\{\begin{array}{llll}
-1 & \text { se } j=1 & (19 \text { Período }) \\
0 & \text { se } j=2 & \text { (2 } 9 \text { Período) } \\
1 & \text { se } j=3 & \text { (39 Período) }
\end{array}\right. \\
& z_{j}=\left\{\begin{array}{rrrr}
1 & \text { se } j=1 & (19 \text { Período }) \\
-2 & \text { se } j=2 & \text { (2\% Período) } \\
1 & \text { se } j=3 & \text { (3! Período) }
\end{array}\right.
\end{aligned}
$$

que correspondem à aplicação do conceito de polinômios ortogonais para o desmembramento dos componentes lineare qua drātico dos períodos.

Sob essas condiçōes, foram obtidos o sistema de equasōes normais, a solução do sistema e as somas de 
quadrados, as distribuiçōes esperanças mntemáticus dns formas quadráticas, o quadro da análise de variância com as esperanças dos quadrados médios, os critérios para os testes das hipóteses de nulidade usuais e os critérios pa ra comparações múltiplas pelo Método de Tukey.

Pudemos observar, com os resultados obtidos que o modelo adotado reproduziu as fórmulas encontradas na bibliografia para análise de dados em ensaios "switch back", que ele se mostrou adequado para os testes de efe tos de tratamentos, blocos, vacas e interação linear períodos $x$ vacas. No que se refere a efeitos de períodos 0 teste F obtido através desse modelo é apenas aproximado. 
ON A LINEAR MODEL TO SWITCHBACK TRIALS IN DESIGNED RAMDOMIZED BLOCKS

Author:

Maria Angélica Ferraz de Toledo Machado

Adviser:

Dr. Antonio Francisco Iemma

S U M M A R Y

The objective of this work was to develop a linear model for switchback trials where the block effect is present. The following model was used:

$$
\begin{aligned}
y_{i j k \ell}=m & +b_{\ell} z_{j}+v_{i}+t_{k}+\alpha_{1} x_{j}+\alpha_{2} z_{j}+c_{i} x_{j}+ \\
& +e_{i j k \ell}
\end{aligned}
$$

where for $i=1,2, \ldots, v ; j=1,2, \ldots, J ; k=1,2, \ldots, p ; \ell=1,2, \ldots$ $\ldots L$, and

$y_{i j k l}$ is the observed value for the $i-t h$ cow in the $j-$ th period, under the k-th treatment of the l-th block;

m - overall mean;

$b_{\ell}-b l o c k \underline{\ell}$ effect; 


$$
\begin{aligned}
& v_{i}-\operatorname{cow} \underline{i} \text { effect; } \\
& t_{k} \text { - treatment } \underline{k} \text { effect; } \\
& \alpha_{1} \text { - period linear effect; } \\
& \alpha_{2} \text { - period quadratic effect; } \\
& c_{i} \text { - period (linear) } x \text { cows interaction effect; } \\
& e_{i j k \ell} \text { - experimental error, assuming } e_{i j k \ell} \cap N\left(0,0^{2}\right) \\
& \text { and that all experimental errors are indepen- } \\
& \text { dent, and } \\
& x_{j}=\left\{\begin{array}{rrrr}
-1 & \text { if } & j=1 & \left(1^{\text {st }} \text { Period }\right) \\
0 & \text { if } & j=2 & \left(2^{\text {sd }} \text { Period }\right) \\
+1 & \text { if } & j=3 & \left(3^{\text {rd }} \text { Period }\right)
\end{array}\right. \\
& z_{j}=\left\{\begin{array}{cccc}
1 & \text { if } & j=1 & \left(1^{\text {st }} \text { Period }\right) \\
-2 & \text { if } & j=2 & \left(2^{\text {sd }} \text { Period }\right) \\
1 & \text { if } & j=3 & \left(3^{\text {rd }} \text { Period }\right)
\end{array}\right.
\end{aligned}
$$

which correspond to the orthogonal polinomial concept on the separation of the periods' linear and quadratic components. Under these conditions the following parameters were obtained: a system its solution and the sum of squares, the distribution and mathematical expectations, the table of analysis of variance along with the mean squares expectations, the criteria to test the null hypothesis and the criteria for multiple comparisons through the use of 
the Tukey's test.

The results obtained showed that the model adopted reproduced the formulae found in the literature for the data analysis of switchback trials, and also that it was adequate for testing treatments, blocks, cows and linear interaction between periods and cows effects. The F test for testing period effects obtained from this model was a somewhat approximate value. 
1. INTRODUÇAOO

Nos experimentos com vacas em lactação, os ensaios de dupla reversão, "switchback" são de grande utí lidade, pois permitem que, para a comparação de um dado número de tratamentos, o pesquisador faça uso de uma quan tidade menor de animais do que aquela tradicionalmente ne cessária em outros esquemas experimentais.

Assim, por exemplo, para a comparação de efe $\underline{i}$ tos de três tratamentos em três repetiçōes são necessārios nove animais para os experimentos delineados inteiramente ao acaso, em blocos casualizados ou em quadrados latinos; enquanto que a mesma comparação pode requerer apenas seis animais se o pesquisador adotar o esquema "switchback". Naturalmente, é redundante ressaltar a impor 
tância da redução do número de animais necessários parase testar um conjunto de hipótesesilde interesse, principalmente no caso de animais de grande porte e de alto vador, como as vacas leiteiras de boa qualidade. Esse fato, por si só, pode ser um bom indicador para o uso de tais ensaios.

O esquema apresentado a seguir ilustra um en saio em "switchback" com três tratamentos.

ESQUEMA COMPLETO

\begin{tabular}{|c|ccc|}
\hline \multirow{3}{*}{ VACAS } & \multicolumn{3}{|c|}{ P E R I O D O S } \\
\cline { 2 - 4 } & I & I I & I I I \\
\hline 1 & A & B & A \\
2 & B & C & B \\
3 & C & A & C \\
4 & A & C & A \\
5 & B & A & B \\
6 & C & B & C \\
\hline
\end{tabular}

Podemos também, quando o número de tratamentos é impar igual ou maior que 5, utilizar os esquems rẹ duzidos, que requerem somente metade das scquências cxịg das pelo esquema completo (LUCAS, 1956). 
Além desse fato, existe o prọblema inerente à influência que a curva de lactação exerce sobre os efệ tos de tratamentos. Este nos parece ser o ponto alto da questão e aqui está patenteado o real valor das idéias de BRANDT (1938) e LUCAS (1956), pois o "switchback" permite reduzir sensivelmente os efeitos dessas influências. Ademais, segundo GODOI (1971), a interação período (linear) $x$ vacas parte do resíduo da análise de variância apresenta "bias" (tendenciosidade), decorrente do fato, já discutido por Lucas (1958) e pimentel Gomes (1960), de que às grandes produções correspondem grandes declínios, levando à super-estimação de $\sigma^{2}$. Os ensaios em "switchback" vem corrigir essa tendenciosidade da variân cia residual, no entanto, possuem uma restrição séria de corrente da perda de graus de liberdade do residuo de $(v-1)$, onde $\underline{v}$, corresponde ao nümero de vacas envolvidas.

Por outro lado, apesar do uso exaustivo dos esquemas em "switchback" no domínio da experimentação agropecuária e recentemente em diversas áreas da medićina humana, praticamente nã் existem na bibliografia disponí vel, estudos sobre aspectos importantes do ponto de vista da inferência estatística.

Um passo significativo nesse sentido foi a- 
presentado por GODOI (1971), que propõe e discute o uso de dois modelos: simplificado e completo, deduzindo as expressōes para' as somas de quadrados.

Em seu trabalho, o autor apresenta, embora sem discutir, um modelo que inclui o efeito de blocos.

Este fato, aliado às necessidades surgidas em pesquisas desenvolvidas no Departamento de Zootecnia da Escola Superior de Agricultura "Luiz de Queiroz", motivou-nos ao estudo detalhado de um modelo linear para ensaios em "switchback", no qual os efeitos de blocos es tivessem presentes.

Assim, temos como objetivos, discutir do pon to de vista estatístico, certos detalhes como as pressuposições do modelo, distribuiçōes e esperanças matemătí cas das formas quadráticas e suas implicações na inferên cia estatística, dentre outros, e apresentar conclusōes que possam ser interpretadas e adotadas por pesquisadores da àrea animal. 


\section{REVISAO BIBLIOGRAFICA .}

\section{1 - Generalidades}

Segundo GILL (1978), os ensaịos de reversão devem ser utilizados quando os efeitos de períodos são grandes e variam de um animal para outro, como por exemplo, a curva de lactação que varia de acordo com a habilidade genética do animal. São eles caracterizados pela aplicação de um ou mais tratamentos duas vezes ao mesmo animal. Para dois tratamentos, segundo esse autor, podem ser utilizados dois tipos de sequências de reversão:

a) Sequências simples de reversão ( $A B A$ e $B A B$ ) se as tendências são lineares, como para produção de leite depois de 4-6 semanas de lactação; 
b) Sequências duplas de reversão ( $A B A B$ e $B A B A$ ), se as tendências são aproximadamente de forma quadrática.Por exemplo, em experimentns com gado de leite, algumas vacas podem estar no início da lactação, quando a produção de leite tende a seguir uma curva convexa com o tempo.

\section{2 - Modelo Matemático}

Nos artigos fundamentais sobre experimentos de reversã̃o apresentados por BRANDT (1938) e LUCAS (1956) não se encontram deduções da anālise de variância nem sugestões de modelos matemáticos e as fórmulas são sempre apresentadas sem qualquer demonstração.

Com a finalidade de obter um modelo matemăti co adequado, que através de deduçōes teóricas reproduzissem os resultados apresentados por BRANDT (1938) e LUCAS (1956), GODOI (1971) estudou dois modelos matemáticos. Em primeiro lugar esse autor estudou o modelo simplificado ou reduzido apresentado a seguir:

$$
y_{i j k}=m+v_{i}+p_{j}+t_{k}+e_{i j k},
$$

onde,

$$
\begin{aligned}
m & =\text { média; } \\
v_{i} & =\text { efeito da vaca } i ; \\
p_{j} & =\text { efeito do período } j ;
\end{aligned}
$$




$$
\begin{aligned}
{ }_{i} t_{k}= & \text { efeito do tratamento } k_{i} \\
e_{i j k}= & \text { erro aleatório onde se supõe } e_{i j k} \cap N\left(0, \sigma^{2}\right) e \\
& \text { independentes; }
\end{aligned}
$$

para o esquema completo com três tratamentos e para o esquema reduzido com cinco tratamentos. Concluiu ele que o modelo simplificado resolve a dedução da análise estatística do delineamento "switchback", porém, o resíduo decor rente desse modelo apresenta tendenciosidade. Esse "bias" decorre do efeito de interação período (linear) $x$ vacas não isolado pelo modelo.

Para eliminar essa tendenciosidade, GODOI

(1971) propõe o modelo completo:

$$
y_{i j k}=m_{-}+v_{i}+t_{k}+b_{\underline{1}} x_{j}+b_{2} z_{j}+c_{i} x_{j}+e_{i j k},
$$

onde :

$$
\begin{aligned}
\mathrm{m} & =\text { média; } \\
\mathrm{v}_{\mathrm{i}} & =\text { efeito da vaca } \mathrm{i} ; \\
\mathrm{t}_{\mathrm{k}}= & \text { efeito do tratamento } \mathrm{k} ; \\
\mathrm{b}_{1}= & \text { efeito linear do período; } \\
\mathrm{b}_{2}= & \text { efeito quadrātico do período; } \\
\mathrm{c}_{\mathrm{i}}= & \text { efeito da interação periodo (1inear) } \mathrm{x} \text { vacas; } \\
\mathrm{e}_{\mathrm{ijk}}= & \text { erro com distribuição normal de média zero e va- } \\
& \text { riância } \sigma^{2} ;
\end{aligned}
$$




$$
\begin{aligned}
& \mathbf{x}_{\mathbf{j}}=\left\{\begin{array}{ccc}
-1 & \text { se } & j=1 \\
0 & \text { se } & j=2 \\
1 & \text { se } & j=3
\end{array}\right. \\
& \mathbf{z}_{j}=\left\{\begin{array}{ccc}
1 & \text { se } & j=1 \\
-2 & \text { se } & j=2 \\
1 & \text { se } & j=3
\end{array}\right.
\end{aligned}
$$

através do qual ele consegue separar o resíduo do modelo simplificado em duas partes: linear e quadrática. Mostrando, então, ser o modelo completo adequado para o "switchback" .

GILL (1981) apresentou também un modelo matemātico para ensaios em "switchback", para dois tratamentos e três períodos:

$$
y_{i j k}=\mu+D_{i}+p_{j}+\tau_{k}+e_{i j k}
$$

que o autor adota também para ensaios em "crossover", onde :

$D_{i}=$ efeito de animal $i=1,2, \ldots r=$ número de animais;

$p_{j}=$ efeito de período $j=1,2,3$;

$\tau_{k}=$ efeito de tratamento $k=1,2$; e 
2.3 - Sistema de Equa ções Normais

GODOI (1971) obteve o sistema de equações nor mais a partir do modelo:

$$
y=X \beta+\because \varepsilon,
$$

onde :

$$
\begin{aligned}
y= & \text { vetor das observações; } \\
X= & \text { matriz do delineamento; } \\
\beta= & \text { vetor dos parâmetros; } \\
\varepsilon= & \text { vetor dos erros, onde se pressupõe que os } e_{i j k} \\
& \text { são independentes e com distribuição normal de } \\
& \text { média zero e variância } \sigma^{2} .
\end{aligned}
$$

A metodologia usada baseia-se no método dos mínimos quadrados (GRAYBILL, 1961), que através da minimi zação da variável $\varepsilon^{\prime} \varepsilon$ resulta no sistema

$$
X^{\prime} X \hat{\beta}=X^{\prime} y .
$$

Como em esquemas experimentais desse tipo,o sistema acima não tem solução única, o autor: apresentou uma matriz de restrições, A, de tal forma que

$$
\text { c }\left(\left[\frac{\mathrm{X}}{\mathrm{A}}-\right]\right)=\alpha \text {, }
$$

onde :

$$
\begin{aligned}
& c=\text { posto } \\
& \alpha=\text { número de parâmetros } .
\end{aligned}
$$


Dessa maneira, o sistema

$$
\left(X^{\prime} X+A^{\prime} A\right) \hat{B}=X^{\prime} Y,
$$

tem solução única, portanto:

$$
\hat{\beta}=\left(X^{\prime} X+A^{\prime} A\right)^{-1} X^{\prime} Y
$$

Essa matriz de restrição tem sido de grande utilidade para facilitar o emprego do computador na obtenção da solução do sistema.

Dentro desse contexto, segundo SEARLE (1971), todos os parâmetros são estimáveis e também todas as funções lineares desses parâmetros, pois a matriz ( $\left.X^{\prime} X+A^{\prime} A\right)$ é não-singular e seu posto é igual ao número de parâmetros do vetor $\beta$.

Uma outra maneira de se obter a solução desse sistema, segundo SCHEFFE (1959), seria através do uso das inversas generalizadas. Caso em que a solução do sistema de equações não é única, portanto os parâmetros não são estimáveis mas sim algumas funçōes lineares deles. 
2.4 - Solução do Sistema de Equaçōes Normais, Análise de Variância e Comparaçōes Mültiplas

Student, em 1908, citado por GODOI (1971), apresentou pela primeira vez uma prova de significância para as médias das diferenças.

BRANDT (1938) apresentou uma extensão do tes te $\underline{t}$ de "Student" para a comparação de médias de dois tra tamentos em ensaios de reversão ou "switchback" envolven do dois, três, quatro ou mais períodos testes.

Em um experimento envolvendo três períodos testes, por exemplo, foram utilizados dois grupos de an $\underline{\underline{i}}$ mais, A e B, para testar dois tratamentos, X e Y, simultaneamente na ordem XYX no grupo A e YXY no grupo B. Para cada animal foram computadas as diferenças

$$
D_{i}=y_{i_{1}}-2 y_{i_{2}}+y_{i_{3}},
$$

onde $i=1,2, \ldots, v$ e $v=$ número de animais. Foram calculadas para cada grupo: a média e a variância dos $D_{i}$ e então obtida a variável para o teste de significância

$$
t=\frac{D_{A}-\bar{D}_{B}}{s} \sqrt{\frac{\left(n_{A}\right)\left(n_{B}\right)}{n_{A}+n_{B}}}
$$

com $n_{A}+n_{B}-2$ graus de 1 iberdade, onde: 


$$
\begin{aligned}
\bar{D}_{A}= & \text { média dos } D_{i} \text { do grupo } A ; \\
D_{B}= & \text { média dos } D_{i} \text { do grupo } B ; \\
n_{A}= & \text { nümero de animais do grupo } A ; \\
n_{B}= & \text { número de animais do grupo } B ; e \\
s= & \sqrt{\frac{\left(n_{A}-1\right) s_{A}^{2}+\left(n_{B}-1\right) s_{B}^{2}}{n_{A}+n_{B}-2}} \\
& \operatorname{com~} s_{A}^{2}=\text { variância dos } D_{i} \text { do grupo } A ; \\
s_{B}^{2}= & \text { variância dos } D_{i} \text { do grupo } B .
\end{aligned}
$$

Após a obtenção do valor da estatística $t$, foi feita a comparação com o valor de $\underline{t}$ da tabela propos ta por Fisher. De modo análogo, o autor mostrou a comparação entre dois tratamentos envolvendo dois e quatro pe ríodos testes.

Nesse trabalho, BRANDT (1938) apresentou, em bora sem deduções, a análise de variância para ensaios de reversão ou "switchback" comparando dois tratamentos em 2,3 e 4 períodos testes. Os resultados descritos abaixo para ilustrar a análise de variância proposta, foram obtidos em um ensaio de alimentaş̃o para 10 vacas em lact ação.

0 número de graus de liberdade total : apresentado foi $3 v-1$ onde $\underline{v}$ é o número de animais testados. 
A subdivisão proposta para esses graus de $1 \underline{i}$ berdade, especificando a estrutura do experimento, foi a seguinte:

Causas de Variação

G.L.

Período

Termo linear $\mathrm{P}_{1} \mathrm{l}$

Termo quadrātico $\mathrm{P}_{2} 1$

Individuais

Vacas do Grupo A $\mathrm{C}_{\mathrm{A}} \mathrm{A}$

Vacas do Grupo B $\mathrm{C}_{\mathrm{B}} \quad \mathrm{A}$

Grupo

G 1

Interaçōes

Entre o temo linear e vacas no Grupo A $\mathrm{P}_{1} \times \mathrm{C}_{\mathrm{A}}$

Entre o temo linear e vacas no Grupo B $\mathrm{P}_{1} \times \mathrm{C}_{\mathrm{B}}$

Entre o termo linear e Grupo $P_{1} \times G \quad l$

Interasōes

Ent re o termo quadrático e vacas no Grupo A $\mathrm{P}_{2} \times \mathrm{C}_{\mathrm{A}}$

Ent re o termo quadrätico e vacas no Grupo B $\mathrm{P}_{2} \times \mathrm{C}_{\mathrm{B}}+$

Entre o termo quadrätico e Grupo $\mathrm{P}_{2} \times \mathrm{G} \quad \mathrm{I}$ 
Para a obtenção das correspondentes somas de quadrados, BRANDT (1938) apresentou os seguintes resujtados:

SOMA DE QUADRADOS TOTAL

$\left(\begin{array}{c}\text { Soma do quadrado de } \\ \text { cada observação }\end{array}\right)-\frac{(\text { soma das observações })^{2}}{\text { nummero total de observaşōes }}$

SOMA DE QUADRADOS PARA PERIODOS

Segundo esse autor, desde que haja 5 períodos experimentais existem duas comparações independentes, ou graus de liberdade entre totais de períodos. Para comparar o total do primeiro período com o total do terceiro período há 1 grau de liberdade, e para comparar a soma dos totais do primeiro e terceiro períodos com duss veres o total do segundo período tem-se também 1 grau de liherdade. O primeiro desses dois graus de liberdade podo sor designado como o termo linear e o segundo como o termo ylus drático:

A soma de quadrados para o termo lines. ( ${ }_{2}$ ) é uma fração do quadrado da diferença entre os totais do 19 e jọ períodos. O denominador da fração é o produto do 
número de observaçoes dentro de cada total de período $c$ a soma dos quadrados dos coeficientes dos termos do numerndor.

$$
P_{1}=\frac{\text { (Total do } 19 \text { Período }- \text { Total do 39 Período) }}{\text { (No de animais) }\left[(1)^{2}+(-1)^{2}\right]}
$$

A soma de quadrados para o tcrmo quadrático $\left(P_{2}\right)$ é uma fração do quadrado da diferenşa entre os totais do 19 e 39 períodos e duas vezes o total do 2 perío do. O denominador da fração é o produto do no de observiçōes dentro de cada total de período e a soma dos quadrados dos coeficientes dos termos do numerador

$$
P_{2}=\frac{[(\text { Total do } 19 P+\text { Total do } 39 P)-2(\text { Total do } 29 P)]^{2}}{\text { aNo de animais })\left[(1)^{2}+(1)^{2}+(-2)^{2}\right]}
$$

SOMA DE QUADRADOS PARA VACAS DENTRO DO GRUPO A

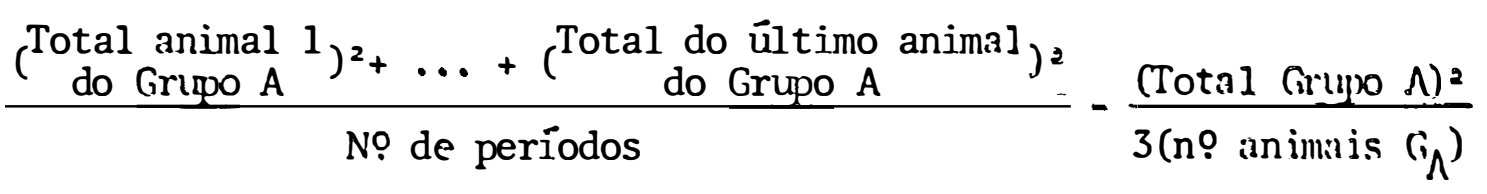

SOMA DE QUADRADOS PARA VACAS DENTRO DO GRUPO B

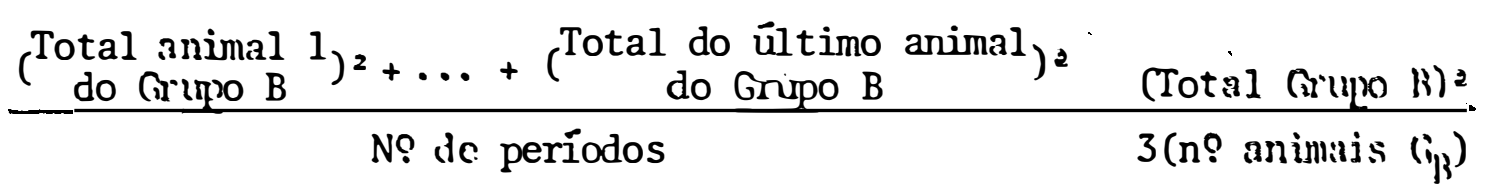


SOMA DE QUADRADOS PARA GRUPOS

\begin{tabular}{cc} 
(Total Grupo A $)^{2}+(\text { Total Grupo B })^{2}$ & (Total Geral)a \\
\hline $\begin{array}{c}\text { No total de observações de cada } \\
\text { grupo }\end{array}$ & No total de observasōes
\end{tabular}

INTERAQOES

As porções da soma de quadrados total atribuídas para as värias interações foram calculadas baseadas an diferenças, como a seguir.

SONA DE QUADRADOSDA INTERACĀO PERTODO LINEAR $\times$ VACAS DO GINUPO A

$$
\frac{1}{\left[(1)^{2}+(-1)^{2}\right]}\left\{\begin{array}{c}
\left(y_{11 k}-y_{13 k}\right)^{2}+\left(y_{21 k}-y_{23 k}\right)^{2}+\cdots+\left(y_{r_{A}} j_{k}-y_{r_{A}{ }^{3 k}}\right)^{2}- \\
-\frac{\left[\sum_{i j}\left(y_{i_{1} k}-y_{i s k}\right)\right]^{2}}{\text { No de animais do } G_{A}}
\end{array}\right\},
$$

onde,

$y_{i j k}$ é o valor observado para o i-ésimo animal no j-êsimo perio do recebendo o k-ésimo tratamento;

$i=1,2, \ldots, r_{A}$ para o Grupo $A, i=1,2, \ldots, r_{B}$ para o Crupo B;

$\mathrm{j}=1,2,3$;

$\mathrm{k}=1,2$. 
SOMA DE QUADRADOSDA INTERACAO PERIODO LINEAR $\times$ VACAS DO GIRUIO B

$$
\frac{1}{\left[(1)^{2}+(-1)^{2}\right]}\left\{\begin{array}{c}
\left(y_{11 k}-y_{13 k}\right)^{2}+\left(y_{21 k}-y_{23 k}\right)^{2}+\cdots+\left(y_{r_{B} 1 k}-y_{r_{B} 3 k}\right)^{2}- \\
-\frac{\left[\sum\left(y_{i 1 k}-y_{i 3 k}\right)\right]^{2}}{\text { No de aninais do } G_{B}}
\end{array}\right\},
$$

SOMA DE QUADRADOS DA INTERAÇĀO PERIODO LINEAR E GRUPO

$$
\frac{\left[\sum_{i=1}^{r_{A}}\left(y_{i_{1 k}}-y_{i_{3 k}}\right)\right]^{2}+\left[\sum_{i=1}^{r_{B}}\left(y_{i_{1 k}}-y_{i_{3 k}}\right)\right]^{2}-\left[\sum_{i=1}^{r_{A}+r_{B}}\left(y_{i_{1 k}}-y_{i_{3 k}}\right)\right]^{2} / 2}{\text { No de animais dentro do grupo }\left[(1)^{2}+(-1)^{2}\right]}
$$

SOMA DE QUADRADOG:DA INTERAÇAO ENTRE O TERMO QUINDRATTIOO E V'ACAS ID GRUPO A

$$
\frac{\sum_{i=1}^{r_{A}} D_{i}^{2}-\left(\sum_{i=1}^{r_{A}} D_{i}\right)^{2} / \text { No de animais do Grupo A }}{\left[(1)^{2}+(-2)^{2}+(1)^{2}\right]}
$$

SOMA DE QUADRADO DA INTERACAO ENTRE O TERMO QUUNDATTTIOO E VACAS DO GRUPO B

$$
\frac{\sum_{i=1}^{r_{B}} D_{i}^{2}-\left(\sum_{i=1}^{r_{B}} D_{i}\right)=/ \text { No de animais do Grujo } B}{\left[(1)^{2}+(-2)=+(1)^{2}\right]}
$$


SOMA DE QUADRADOS DA INTERACAO ENTRE O TERAD QUADRATIDO E GRUTO

$$
\frac{\left(\sum_{i=1}^{r_{A}} D_{i}\right)^{2}+\left(\sum_{i=1}^{r_{B}} D_{i}\right)^{2}-\left(\sum_{i=1}^{r_{A}} D_{i}+\sum_{i=1}^{r_{B}} D_{i}\right)^{2} / 2}{\text { No de animais dentro do grupo }\left[(1)^{2}+(-2)^{2}+(1)^{2}\right]}
$$

Ainda segundo BRANDT (1938), devido às carac terísticas do delineamento de reversāo ou "switchback", o efeito de tratamento é idêntico ao efeito da interaşào en tre o termo quadrático e grupo. Ele demonstra que isso $\bar{e}$ facilmente observado pela tabela abaixo.

\begin{tabular}{ccc}
\multicolumn{3}{c}{ PERIODOS } \\
\hline TERMO QUADRATICO \\
\hline GRUPO & I + III & $2($ II $)$ \\
A & $X_{1}+X_{3}$ & $2 Y_{2}$ \\
B & $Y_{2}+Y_{3}$ & $2 X_{2}$ \\
\hline
\end{tabular}

onde:

$$
\begin{aligned}
& x_{1} \text { é o tratamento } x \text { no } 19 \text { periodo; } \\
& x_{2} \text { é o tratamento } x \text { no } 29 \text { periodo; } \\
& x_{3} \text { é o tratamento } x \text { no } 50 \text { periodo; e } \\
& y \text { de modo análogo. }
\end{aligned}
$$


A interação contre o termo quadrítico e grupo é a comparação diagonal da tabcla mencionada acima, que função da expressão $\left(X_{1}-2 X_{2}+X_{3}\right)-\left(Y_{1}-2 Y_{2}+Y_{3}\right) \cdot$ Is: sa expressão é também a diferença entre os resultados obtidos das rações $X$ e $Y$ adequadamente ponderada para uso eficiente de todas as informações disponíveis.

Nesse experimento, devido ao clolineamonto, existe uma relevante questão: A significância da variahilidade devida ao tratamento, como foi mostrado, é a mcsima para interação entre termo quadrático e grupo. A estimati va apropriada para o erro, quando se testa a significincial dessa interação, é obtida juntando-se a interaşio entro tọ mos quadräticos e os grupos.

\begin{tabular}{|c|c|c|c|c|}
\hline & G.L. & S.Q. & Q.M. & $\mathrm{F}$ \\
\hline $\mathrm{P}_{2} \times \mathrm{G}_{\mathrm{A}}$ & 4 & & & \\
\hline $\mathrm{P}_{2} \times \mathrm{G}_{\mathrm{B}}$ & 4 & & & \\
\hline Erro (Soma) & 8 & SQ Erro & SQ Erro/8 & \\
\hline$P_{2} \times G$ & 1 & SQ $\left(P_{2} x G\right)$ & {$[S)\left(P_{2} \times(i)\right] / 1$} & {$\left[(N)\left(P_{2} \times(i)\right) / N M\right.$ larro } \\
\hline
\end{tabular}

$S Q$ Erro $=S Q\left(P=\times G_{A}\right)+S Q\left(P_{2} \times G_{B}\right)$

0 autor apresentou também a análise de covariância para uma e duas variáveis concomitantes. 
Seath (1944), citado por LUCAS; (1956), llinle: jou um dclineamento em "switchback" com quatro sequências para estudar um fatoria $12 \times 2$. O delineamento forneceu um teste sensivel para dois efeitos principais, mas produziu um teste pobre para a interação.

Snedecor (1946), citado por GODOI (1971), tium bém descreveu uma eficiente. análise estatística para onsaios "switchback".

PATTERSON (1950) e LUCAS (1951), trabalhando com ensaios rotacionais cos ensaios rotacionais se caracterizam pelo fato de cada animal receber tratamentos distintos, isto é, não existe repetição de tratamento durante a realização do ensaio), chegaram ambos a resultados mostrando tendenciosidade na estimativa da variância resi dual nesses ensaios.

o "bias" é captado quando se desdobra a ririância nos componentes linear e quadrático.

TAYLOR e AMSTRONG (1953) mostraram que o delineamento "switchback" pode ser estendido para qualquer número de tratamentos e tem resumidamente indicado o mito do de anälise. Introduziram os delineamentos reduzidos, is to é, delineamentos nos quais cada combinação de tratame!l tos aparece em uma sequência apenas, ao invés de duas, co mo ocorre nos delineamentos completos. 
LUCAS (1956) ressaltou a sensibilidade de en saios de reversāo quando se faz pesquisa com gado de leite. Basicamente, entretanto, esses delineamentos permitiam a comparação de somente dois tratamentos, e isso tem imposto alguma limitação na sua utilidade.

0 autor dedicou seu trabalho para delineamen tos em "switchback" que permitam comparações de três ou mais tratamentos.

O delineamento básico de "switchback" apresentado no trabalho, foi o seguinte:

\begin{tabular}{ccc}
\hline PERIODO EXPERIMENTAL & $\cdots$ & TRATANENTO-SEQUENCIA \\
& 1 & 2 \\
\hline 1 & 1 & 2 \\
2 & 2 & 1 \\
3 & 1 & 2 \\
\hline
\end{tabular}

Segundo o autor, o esquema para tratamentos resulta em comparaçōes particularmente sensíveis, porque ele permite eliminar do erro residual:

a) efeito de períodos derido a mudanças no ambiente;

b) Variação no nível de proiucão entre vacas; e

c) a maior parte da variasĩo entre vacas quanto ao grau de inclinação da curva de lactação. 
Ele citou a análise estatística do Brandt (1938) e o teste para comparação de dois tratamentos.

0 autor mostrou que a comparação de p tratamentos requer $p(p-1)$ sequências de tratamentos(delineamen to completo). Se pé ímpar e igual a 5 ou maior, entretan to, delineamentos que requerem somente $p(p-1) / 2$ sequôncias podem ser usadas (delineamento reduzido). O autor apresentou uma seleção de esquemas padrões para delineamcn tos completos (com 3, 4 e 6 tratamentos) e redusidos (com 5,7 e 9 tratamentos). Os delineamentos completos foram subdivididos dentro de p-l blocos de p sequênias cada, e o delineamento reduzido dentro de $(p-1) / 2$ blocos de $p$ sequências cada. Segundo o autor, para um erro experimental mínimo, as vacas designadas para o esquema devem ter passado do pico da lactação antes de iniciar o experimento e nenhum animal deverá passar do meio da gestaçio até o fí nal do ensaio. Sempre que o número de animais que satisfazem essas condições não é suficiente para iniciar todas as sequências do delineamento ao mesmo tomlro, podcm ser utilizados blocos.

0 autor apresentou a seguinte anílise cstatística. Preliminarmente foram computadas para cada vaca, a quantidade: $D=Y_{1}-2 Y_{2}+Y_{3}$, no qual $Y_{1}$, J: $Y_{3}$ representam os valores observados nos períodos 1, 2 e 3 res 
pectivamente. 0 passo seguinte foi computar:

$$
\begin{aligned}
M= & \text { soma dos } D^{\prime} \text { s para todas as vacas em todas as sc- } \\
& \text { quências, e se o delineanento é cm blocos, calcu } \\
& \text { la-se para cada bloco; } \\
B= & \text { soma dos D's no bloco. }
\end{aligned}
$$

No terceiro passo foi calculado para cada trạ tamento:

$$
\begin{aligned}
Q= & \text { soma dos D's para vacas recebendo o tratamento no } \\
& 19 \text { e } 39 \text { períodos, menos a soma dos D's para va- } \\
& \text { cas recebendo o tratamento no } 29 \text { período. }
\end{aligned}
$$

PONTES DE VARIAC,AO

G.L.
SOMAA DE QUINIIR:DOS Abreviatura
Fónmulars

C.F.

$\frac{M^{2}}{3 n p(p-1)}$

T.S.S. $\quad \frac{1}{6} \sum_{i j} \sum_{j} D_{i j}^{2}-C F$

Total (corrigido)

$\frac{\mathrm{np}(\mathrm{p}-1)}{2}-1$

S.S.B. $\frac{1}{o} \sum_{u} \frac{B_{u}^{2}}{m_{u}}-C F$

Blocos (se presente)

$v-1$

$\mathrm{p}-1$

S.S.T. $\frac{1}{\operatorname{comp}} \sum_{\mathrm{k}} \mathrm{Q}_{\mathrm{k}}^{2}$

Erro (hlocos presentes) $\frac{\mathrm{np}(\mathrm{p}-1)-2(\mathrm{p}+\mathrm{v})+2}{2}$

S.S.E.

T'SS - SSB - SST

Erro (sem blocos)

$$
n \mathrm{p}^{2} \div \frac{(\mathrm{n}+2) \mathrm{p}}{2}
$$

TSS - SST 
O divisor 6 que aparece nas fórmulas das somas de quadrados, segundo o autor, é usado para colocar as somas de quadrados em uma base por vaca e por período.

$$
\begin{aligned}
& \mathrm{p}=\mathrm{n} \rho \text { de tratamentos; } \\
& \mathrm{n}=\left\{\begin{array}{r}
\mathrm{r} \text { em delineamentos reduzidos; } \\
2 \mathrm{r} \text { em delineamentos completos; }
\end{array}\right.
\end{aligned}
$$

onde $\mathrm{r}=\mathrm{n}$ ? de vacas por sequência de tratamentos;

$$
\begin{aligned}
v= & n o \text { de blocos no delineamento; } \\
m_{u}= & n \text { o de vacas no u-ésimo bloco; } \\
D_{i j}= & \text { valor D para a j-ésima vaca na i-ésima sequência } \\
& \text { de tratamento; } \\
B_{u}= & \text { valor B para o u-ésimo bloco; } \\
Q_{k}= & \text { valor Q para o k-ésimo tratamento. }
\end{aligned}
$$

Os quadrados médios podem ser computados e o teste $F$ para tratamentos feito de modo usual.

Média de Tratamento: A média para o k-ésimo tratamento foi obtida como

$$
\overline{\overline{\mathrm{Y}}}+\mathrm{Q}_{\mathrm{k}} / 2 \mathrm{np},
$$

no qual İ é a média geral das produções no experimento, is to é, a média dos dados originais, não a média dos D's.

0 erro padrão da diferença de tratamenios apre sentado pelo autor foi $\sqrt{3 s^{2} / n p}$, onde $s^{2}=$ QMR.

0 autor apresentou ainda a anäliso de $\underline{v}$. 
riância quando existem dados perdidos ou anormais, pois ocasionalmente uma vaca pode iniciar o experimento e não terminar, ou sua produção durante um ou mais jeríodos experimentais pode ser anormal devido a fatores não contro láveis. Quando isso ocorre é necessário ajustar um valor D, que viabiliza a análise de variância. Se o delineamento é em blocos, o valor perdido D é obtido pela fórmula:

$$
D=\frac{n p B_{s}^{\prime}+m_{s}\left(Q_{s f}^{\prime}-Q_{s c}^{\prime}\right)}{n p\left(m_{s}-1\right)-2 m_{s}}
$$

onde $\mathrm{n}$ e $\mathrm{p}$ são definidos como anteriormente $\mathrm{c}$ :

$$
\begin{aligned}
& \mathrm{m}_{\mathrm{s}}=\mathrm{n} \text { e de vacas no bloco onde o valor perdido ocor- } \\
& \mathrm{B}_{\mathrm{S}}^{\prime}=\text { soma dos } \underline{\mathrm{D}} \text { referentes aos valores nīo perdidos } \\
& Q_{S f}^{\prime}=\text { valor } Q \text { para o tratamento que ocorre no } 10 \text { e } 3 \text { ? } \\
& Q_{S C}^{\prime}=\text { valor } Q \text { para o tratamento que ocorre no } 29 \text { pe- }
\end{aligned}
$$

Se o delineamento não é em blocos a fórmula fica:

$$
D=\frac{2 M^{\prime}+(p-1)\left(Q_{s f}^{\prime}-Q_{s c}^{\prime}\right)}{n p^{2}-(n+2) p}
$$


no qual $\mathrm{M}^{\prime}$ = a soma dos $\mathrm{D}^{\prime}$ 's não perdidos, e as outras quan tidades são definidas como anteriormente.

0 autor apresentou tambëm um exemplo no qual os dados simulam um experimento comparando 3 tratamentos com 12 animais; para ilustrar o procedimento em blocos ele assumiu que 6 das vacas iniciam o experimento em um tempo com delineamento completo, e as vacas remanescentes iniciam mais tarde em 2 grupos de 3 , cada grupo formando um bloco. Ele mostrou ainda a análise de variância, as médias dos tratamentos, o erro padrão das diferenças de tra tamentos e também o coeficiente de variação dado por:

$$
\mathrm{CV}=\frac{100 \sqrt{\mathrm{QMR}}}{\hat{\mathrm{m}}}
$$

onde, $\mathrm{CV}=$ coeficiente de variação;

$$
\begin{aligned}
\mathrm{QMR} & =\text { quadrado médio do resíduo; } \\
\hat{\mathrm{m}} & =\text { média geral. }
\end{aligned}
$$

GODOI (1971), após a obtenção da solução do sistema de equações normais, obteve as deduções matemáticas das fórmulas da análise de variância de um delineamen to em "switchback", através dos modelos matemáticos propostos.

0 primeiro modelo utilizado pelo autor foi o modelo matemático simplificado ou reduzido, isto é, 


$$
y_{i j k}=m+v_{i}+p_{j}+t_{k}+e_{i j k},
$$

conforme descrito em 2.2.

0 autor fez o'estudo para esse modelo utilizando dois casos:

CASO I - Esquema completo.

PERIODOS

\begin{tabular}{cccc}
\hline VACAS & $\mathrm{P}_{1}$ & $\mathrm{P}_{2}$ & $\mathrm{P}_{3}$ \\
\hline $\mathrm{v}_{1}$ & $1^{*}$ & 2 & 1 \\
$\mathrm{v}_{2}$ & 2 & 3 & 2 \\
$\mathrm{v}_{3}$ & 3 & 1 & 3 \\
$\mathrm{v}_{4}$ & 1 & 3 & 1 \\
$\mathrm{v}_{5}$ & 2 & 1 & 2 \\
$\mathrm{v}_{6}$ & 3 & 2 & 3 \\
\hline
\end{tabular}

*Os números dentro do quadro (acima), refercml-se aos trata mentos atribuídos às parcelas.

0 autor obteve, como soluçāo do sistema de equaçōes normais, para o esquema em estudo, as seguintes es timativas:

a) Para a média:

$$
\hat{\mathrm{m}}=\frac{\mathrm{G}}{18} \text {, onde } \mathrm{G} \text { é o total geral. }
$$


b) Para efeito de períodos:

$$
\begin{aligned}
& \hat{\mathrm{p}}_{1}=\frac{\mathrm{P}_{1}}{6}-\frac{\mathrm{G}}{18} \\
& \hat{\mathrm{p}}_{2}=\frac{\mathrm{P}_{2}}{6}-\frac{\mathrm{G}}{18} \\
& \hat{\mathrm{p}}_{3}=\frac{\mathrm{P}_{3}}{6}-\frac{\mathrm{G}}{18}
\end{aligned}
$$

onde $P_{j}$ é o total correspondente ao período $j$.

c) Para efeito de vacas:

$$
\begin{aligned}
& \hat{v}_{1}=\frac{1}{3}\left(V_{1}-\frac{3 G}{18}-2 \hat{t}_{1}-\hat{t}_{2}\right) \\
& \hat{v}_{2}=\frac{1}{3}\left(V_{2}-\frac{3 G}{18}-2 \hat{t}_{2}-\hat{t}_{3}\right) \\
& \hat{v}_{3}=\frac{1}{3}\left(V_{3}-\frac{3 G}{18}-\hat{t}_{1}-2 \hat{t}_{3}\right) \\
& \hat{v}_{4}=\frac{1}{3}\left(V_{4}-\frac{3 G}{18}-2 \hat{t}_{1}-\hat{t}_{3}\right) \\
& \hat{v}_{5}=\frac{1}{3}\left(v_{5}-\frac{3 G}{18}-\hat{t}_{1}-2 \hat{t}_{2}\right) \\
& \hat{v}_{6}=\frac{1}{3}\left(v_{6}-\frac{3 G}{18}-\hat{t}_{2}-2 \hat{t}_{3}\right)
\end{aligned}
$$

onde $V_{i}$ é o total correspondente à vaca $\underline{i}$. 
Através das soluçōes do sịstema de equaçōes normais, obteve-se a Soma de Quadrados dos Parâmetros:

$$
\begin{aligned}
\text { SQ Parâmetros }= & \hat{\mathrm{t}}_{1} \mathrm{~T}_{2}+\hat{\mathrm{t}}_{2} \mathrm{~T}_{2}+\hat{\mathrm{t}}_{3} \mathrm{~T}_{3}+\hat{\mathrm{v}}_{1} \mathrm{~V}_{2}+\hat{\mathrm{v}}_{2} \mathrm{~V}_{2}+ \\
& +\ldots+\hat{\mathrm{v}}_{6} \mathrm{~V}_{6}+\hat{\mathrm{p}}_{1} \mathrm{P}_{1}+\hat{\mathrm{p}}_{2} \mathrm{P}_{2}+\hat{\mathrm{p}}_{3} \mathrm{P}_{3}
\end{aligned}
$$

Como os parâmetros de tratamentos e vacas não são independentes, a soma de quadrados de parâmetros foi rearranjada de forma a ficar ortogonalmente partida. Assim,

$$
\begin{aligned}
\mathrm{SQ} \text { Parâmetros }= & \sum_{k=1}^{3} \overline{\mathrm{t}}_{\mathrm{k}} \mathrm{Q}_{\mathrm{k}}^{*}+\left(\frac{1}{3} \sum_{i=1}^{6} \mathrm{~V}_{\mathrm{i}}^{2}-\frac{\mathrm{G}^{2}}{18}\right)+ \\
& +\left(\frac{1}{6} \sum_{j=1}^{3} P_{j}^{2}-\frac{\mathrm{G}^{2}}{18}\right)
\end{aligned}
$$

onde $Q_{k}^{*}=\frac{1}{3} Q_{k}$, e

$$
\begin{aligned}
Q_{k}= & \text { soma dos } D^{\prime} \text { s em que o tratamento } \underline{k} \text { aparece nos } \\
& 19 \text { e } 39 \text { períodos, subtraída da somi dos } D^{\prime} s \text { em } \\
& \text { que o tratamento } \underline{k} \text { aparece no } 20 \text { periodo (def } \underline{\mathrm{i}} \\
& \text { nida por LUCAS, } 1956 \text { ). }
\end{aligned}
$$

Foi tambēm obtida pelo autor a sulução do sistema para efeitos de tratamentos:

$$
\hat{\mathrm{t}}_{\mathrm{k}}=\frac{1}{4} \mathrm{Q}_{\mathrm{k}}^{*} \text {. }
$$


Portanto a Soma de Quadrado de Parâmetros,fi

cou:

$$
\begin{aligned}
& \text { SQ Parâmetros }=\frac{1}{4} \sum_{k=1}^{3} Q_{k}^{2}+\left(\frac{1}{3} \sum_{i=1}^{6} V_{i}^{2}-\frac{G^{2}}{18}\right)+ \\
&+\left(\frac{1}{6} \sum_{j=1}^{3} P_{j}^{2}-\frac{G^{2}}{18}\right) \\
& S Q \text { Parâmetros }=\frac{1}{36} \sum_{k=1}^{3} Q_{k}^{2}+\left(\frac{1}{3} \sum_{i=1}^{6} V_{i}^{2}-\frac{G^{2}}{18}\right)+ \\
& \quad+\left(\frac{1}{6} \sum_{j=1}^{3} P_{j}-\frac{G}{18}\right) \\
& \text { SQ Parâmetros }=S Q \text { Trat. (aj.) }+ \text { SQ Vacas (usual) + } \\
&+S Q \text { Periodos (usual) }
\end{aligned}
$$

Verificou-se que existe uma perfeita identidade das somas de quadrados de tratamentos ajustadas, jus tificando o modelo simplificado para a derivação dessa par te da soma de quadrados total.

As partes correspondentes a vacas e períodos não foram levadas em consideração na análise convencional, apresentada por LUCAS (1956). 
31.

CASO II - Esquema reduzido.

\begin{tabular}{cccc}
\hline & & P E R I O D O S \\
\hline VACAS & I & I I & I I I \\
\hline$v_{1}$ & 1 & 2 & 1 \\
$v_{2}$ & 2 & 3 & 2 \\
$v_{3}$ & 3 & 4 & 3 \\
$v_{4}$ & 4 & 5 & 4 \\
$v_{5}$ & 5 & 1 & 5 \\
$v_{6}$ & 1 & 3 & 1 \\
$v_{7}$ & 2 & 4 & 2 \\
$v_{8}$ & 3 & 5 & 3 \\
$v_{9}$ & 4 & 1 & 4 \\
$v_{10}$ & 5 & 2 & -5 \\
\hline
\end{tabular}

Do mesmo modo como foi feito para o Caso I, o autor obteve a solução do sistema de equaçōes normais:

a) Para a média:

$$
\hat{\mathrm{m}}=\frac{\mathrm{G}}{30}
$$

b) Para efeito de vacas:

$$
\hat{v}_{1}=\frac{1}{3}\left(V_{1}-3 \hat{m}-2 \hat{E}_{1}-\hat{t}_{2}\right)
$$




$$
\begin{aligned}
\hat{v}_{2} & =\frac{1}{3}\left(v_{2}-3 \hat{m}-2 \hat{t}_{2}-\hat{t}_{9}\right) \\
\hat{v}_{3} & =\frac{1}{3}\left(v_{3}-3 \hat{m}-2 \hat{t}_{3}-\hat{t}_{4}\right) \\
\hat{v}_{4} & =\frac{1}{3}\left(V_{4}-3 \hat{m}-2 \hat{t}_{4}-\hat{t}_{5}\right) \\
\hat{v}_{5} & =\frac{1}{3}\left(V_{5}-3 \hat{m}-2 \hat{t}_{5}-\hat{t}_{1}\right) \\
\hat{p}_{3} r a & =\frac{1}{10}\left(P_{3}-10 \hat{m}\right) \\
\hat{v}_{6} & =\frac{1}{3}\left(V_{6}-3 \hat{m}-2 \hat{t}_{1}-\hat{t}_{3}\right) \\
\hat{p}_{1} & =\frac{1}{10}\left(P_{1}-10 \hat{m}\right) \\
\hat{v}_{7} & =\frac{1}{3}\left(v_{7}-3 \hat{m}-2 \hat{t}_{2}-\hat{t}_{4}\right) \\
\hat{v}_{9} & =\frac{1}{3}\left(V_{9}-3 \hat{m}-2 \hat{t}_{4}-\hat{t}_{1}\right) \\
\hat{v}_{8} & =\frac{1}{3}\left(V_{8}-3 \hat{m}-2 \hat{t}_{3}-\hat{t}_{5}\right)
\end{aligned}
$$


A soma de quadrados dos Parâmetros resultou:

$$
\begin{aligned}
\text { SQ Parâmetros }=\hat{m} G & +\hat{E}_{1} T_{1}+\hat{E}_{2} T_{2}+\hat{t}_{3} T_{3}+\hat{v}_{1} V_{1}+\ldots+ \\
& +\hat{v}_{10} V_{10}+\hat{p}_{1} P_{1}+\hat{p}_{2} P_{2}+\hat{p}_{3} p_{3}
\end{aligned}
$$

que, desenvolvida, fica:

$$
\begin{aligned}
\text { SQ Parâmetros }=\sum_{k=1}^{5} \hat{t}_{k} Q_{k}^{*} & +\left(\frac{1}{3} \sum_{i=1}^{10} V_{i}^{2}-\frac{G^{2}}{30}\right)+ \\
& +\left(\frac{1}{10} \sum_{j=1}^{3} P_{j}^{2}-\frac{G^{2}}{30}\right)
\end{aligned}
$$

Pela estrutura do desenvolvimento, o autor chegou à generalização,

$$
\begin{aligned}
& Q_{k}^{*}=T_{k}-\frac{2}{3} \sum_{k} V_{k}^{\prime}-\frac{1}{3} \sum_{k} V_{k}^{\prime \prime} \\
& \hat{t}_{k}=\frac{3}{2 n p} Q_{k}^{*},
\end{aligned}
$$

onde,

$$
\begin{aligned}
\mathrm{V}_{\mathrm{k}}= & \text { total correspondente às vacas que receberam o } \\
& \text { tratamento } \mathrm{k} \text { nos } 19 \text { e } 39 \text { períodos; } \\
\mathrm{V}_{\mathrm{k}}^{\prime \prime}= & \text { total correspondente às vacas que receberam o } \\
& \text { tratamento } \mathrm{k} \text { no } 20 \text { período; } \\
\mathrm{r}= & \text { nümero de repetições de cada sequência; }
\end{aligned}
$$




$$
\mathbf{n}=\left\{\begin{array}{r}
r, \text { se foi esquema reduzido; } \\
2 r, \text { se foi esquema completo }
\end{array}\right.
$$

o autor mostrou ainda um exemplo numérico on de apresentou a análise de variância para o modelo simplí ficado.

\begin{tabular}{lc}
\hline Fonte de Variação & G.L. \\
\hline Vacas `(não aj.) $^{*} \mathrm{v}-1$ \\
Períodos & $\mathrm{j}-1$ \\
Tratamentos & $\mathrm{p}-1$ \\
Resíduo & por diferença \\
\hline Total & $\mathrm{N}-1$ \\
\hline
\end{tabular}

A análise de variância apresentada por Lucas (1956) e citada pelo autor, é:

\begin{tabular}{lccc}
\hline Fonte de Variação & $:$ & G.L. & S.Q. \\
\hline Tratamento $(a j)$. & p-l & SQT \\
Resíduo & por diferença & SQ Res. \\
\hline Total & v-l & SQ Total \\
\hline
\end{tabular}


onde :

$$
\begin{aligned}
S Q \text { Total } & =\frac{1}{6} \Sigma D_{i}^{2}-\frac{1}{3 n p(p-1)}\left(\Sigma D_{i}\right)^{2} \\
S Q \text { Resíduo } & =S Q \text { Total }-S Q \text { Trat. }(a j .)
\end{aligned}
$$

O autor, examinando a composição do resíduo no modelo reduzido mostrou que se pode decompô-lo cm 2 partes aditivas, ou sejam:

$$
\begin{aligned}
& \text { Interação período (efeito linear) x vacas: } v-1 \\
& \text { Interação período (efeito quadratico) } x \text { va } v-p \\
& \text { cas (eliminado efeito de tratamento) }
\end{aligned}
$$

onde o componente linear é obtido pela diferença entre o resíduo do modelo simplificado e o resíduo da análiśc de LUCAS (1956).

\begin{tabular}{lcc}
\hline Fonte de Variação & G.L. & S.Q. \\
\hline$P^{\prime} \times \mathrm{V}$ & $v-1$ & por diferença \\
P' $^{\prime \prime} \mathrm{V}$ & $\mathrm{v}-\mathrm{p}$ & SQ Res. (LUCAS) \\
\hline Total (Res. Mod. Red.) & & SQ Res. (Mod.Red.) \\
\hline
\end{tabular}

Para verificar se os dois componentes csi i mam $\sigma^{2}$, o autor obteve os componentes de variância. 
A seguir, GODOI (1971) introduziu o modelo matemático completo dos ensaios "switchback", isto è, um mòdelo matemático que permitia a estimaçāo de parcelas dẹ dução das somas de quadrados ajustadas e possibilitava a aplicação de esperança matemática às estimativas de médias, variâncias, parcelas perdidas, etc.

0 modelo matemático introduzido foi:

$$
y_{i j k}=m+v_{i}+t_{k}+b_{1} x_{j}+b_{2} z_{j}+c_{i} x_{j}+c_{i j k},
$$

conforme descrito em (2.2).

0 autor demonstrou que o modelo proposto cor responde ao desdobramento do resíduo do modelo reduzido nas partes linear e quadrática, conservanto os demais efeitos inalterados, a não ser o desmembramento feito dos componentes linear e quadrätico dos períodos, através da aplicação do conceito de polinômios ortogonais no modelo reduzido.

Foi admitido um esquema com dois tratamentos, duas repetições por sequência e 6 vacas, como a seguir: 


\begin{tabular}{cccc}
\hline VACAS & $\mathrm{P}_{2}$ & $\mathrm{P}_{2}$ & $\mathrm{P}_{3}$ \\
\hline 1 & 1 & 2 & 1 \\
2 & 1 & 2 & 1 \\
3 & 1 & 2 & 1 \\
4 & 2 & 1 & 2 \\
5 & 2 & 1 & 2 \\
6 & 2 & 1 & 2 \\
\hline
\end{tabular}

Após a montagem da equação matricial, o autor obteve as soluções do sistema:

a) Para efeito linear de período:

$$
\sigma_{1}=\frac{1}{12}\left(P_{3}-P_{1}\right)
$$

b) Para efeito quadrático de período:

$$
G_{2}=\frac{1}{36}\left(P_{1}-2 P_{2}+P_{3}\right)
$$

c) Para efeito da interação (linear) períodos $x$ Vacas :

$$
\hat{c}_{1}=\frac{1}{2}\left(y_{131}-y_{111}\right)-\frac{1}{12}\left(P_{3}-P_{1}\right)
$$


38.

$$
\begin{aligned}
& \hat{c}_{2}=\frac{1}{2}\left(y_{231}-y_{211}\right)-\frac{1}{12}\left(P_{3}-P_{1}\right) \\
& \hat{c}_{3}=\frac{1}{2}\left(y_{331}-y_{311}\right)-\frac{1}{12}\left(P_{3}-P_{1}\right) \\
& \hat{c}_{4}=\frac{1}{2}\left(y_{432}-y_{412}\right)-\frac{1}{12}\left(P_{3}-P_{1}\right) \\
& \hat{c}_{5}=\frac{1}{2}\left(y_{532}-y_{512}\right)-\frac{1}{12}\left(P_{3}-P_{1}\right) \\
& \hat{c}_{6}=\frac{1}{2}\left(y_{632}-y_{612}\right)-\frac{1}{12}\left(P_{3}-P_{1}\right)
\end{aligned}
$$

A solução do sistema de equações normais jara os demais parâmetros é obtida da mesma maneira que no caso do modelo matemático rêluzido.

As fórmulas, para iompletar o quadro de anarlise de variância, para os componentes linear e quadrätjco de períodos e a interação períoto (linear) x vacas, apresentadas foram:

$$
\begin{aligned}
\operatorname{SQ}\left(\bar{b}_{1}, \bar{b}_{2}\right) & =\frac{1}{12}\left(P_{1}-P_{3}\right)^{2}+\frac{1}{36}\left(P_{1}-2 P_{2}+P_{3}\right)^{2} \\
& =\frac{1}{6} \sum_{j=1}^{3} P_{j}^{2}-\frac{G^{2}}{1 s} \\
\operatorname{SQ}\left(\hat{c}_{i}\right) & =\frac{1}{2} \sum_{i}\left(y_{i \leqslant k}-y_{i 2 k}\right)^{2}-\frac{1}{12}\left(P_{1}-P_{3}\right)^{2}
\end{aligned}
$$


0 autor apresenta ainda a generalização:

$$
\begin{aligned}
\operatorname{SQ}\left(\bar{b}_{1}, \bar{b}_{2}\right) & =\frac{1}{v}\left(\sum_{j=1}^{3} P_{j}^{2}\right)-\frac{G^{2}}{3 v} \\
\operatorname{SQ}\left(\hat{c}_{i}\right) & =\frac{1}{2} \sum_{i}\left(y_{i 3 k}-y_{i 1 k}\right)^{2}-\frac{1}{2 v}\left(P_{1}-P_{3}\right)^{2}
\end{aligned}
$$

Combinando os resultados do modelo matemätico reduzido e os do completo, foi obtida a seguinte análi se de variância:

F. de Variação G.L. . S.Q.

Vacas (ign. trat.) $\quad v-1 \quad \frac{1}{3} \sum_{i=1}^{v} V_{i}^{2}-\frac{G^{2}}{3 v}$

Períodos (lânear) ; $1 \quad \frac{1}{2 v}\left(\mathrm{P}_{1}-\mathrm{P}_{3}\right)^{2}$

Períodos (quadr.) $1 \quad \frac{1}{6 \mathrm{~V}}\left(\mathrm{P}_{1}-2 \mathrm{P}_{2}+\mathrm{P}_{3}\right)^{2}$

Tratamentos (aj.) $\quad \mathrm{p}-1 \quad \frac{3}{2 \mathrm{np}} \underset{\mathrm{k}=1}{\mathrm{p}}\left(\mathrm{Q}_{\mathrm{k}}^{*}\right)^{2}$

Int. $P^{\prime} \times V \quad \quad v-1-\frac{1}{2} \underset{i=1}{v}\left(y_{i 3 k}-y_{i \underset{j}{1} k}\right)^{2}-\frac{1}{2 v}\left(P_{1}-P_{3}\right)^{2}$

Resíduo v-p por diferença

Total $3 v-1 \quad \sum_{i, j} y_{i j k}^{2}-\frac{G^{2}}{3 v}$ 
com as seguintes esperanças dos quadrados médios:

\begin{tabular}{lc}
\hline Fontes de Variação & $E(Q . M)$. \\
\hline Vacas (ign. trat.) & $\sigma^{2}+12 b_{1}^{2}$ \\
Periodos (1 inear) & $\sigma^{2}+36 b_{2}^{2}$ \\
Periodos (quadr.) & $\sigma^{2}+2\left(t_{1}-t_{2}\right)^{2}$ \\
Trat. (aj.) & $\sigma^{2}+\frac{2}{5} \sum_{i} c_{i}^{2}$ \\
Interação $P^{\prime} \times v$ & $\sigma^{2}$ \\
Resíduo &
\end{tabular}

GODOI (1971) apresentou também a aplicação do modelo matemático completo no estudo do delineamento cm "switchback" quando se tem perda de parcela.

0 autor discutiu as fórmulas de Lucas (1950) para o cálculo da parcela perdida, e através da esperansa matemática demonstra o "bias" na estimativa do quadraco médio de tratamentos, fato que compromete a aplicação do teste $\mathrm{F}$.

Segundo o autor, uma outra maneira de se dosenvolver a soma de quadrados de tratamentos ajustada, quin do se perde uma ou mais parcelas, poderia ser obtida dodu zindo-se as fórmulas a partir do modelo completo, o quil 
permite o conhecimento do fator de ajustamento.

Para o esquema em estudo, o autor apresentou o contraste entre estimativas de duas médìas, que é dado por:

$$
\hat{c}=\frac{1}{2 n p}\left(Q_{1}-Q_{2}\right),
$$

e a variância da estimativa ĉ, é:

$$
\begin{aligned}
& V(\hat{c})=E\left\{[\hat{c}-E(\hat{c})]^{2}\right\} \\
& V(\hat{c})=\frac{5}{16} \sigma^{2} .
\end{aligned}
$$

Substituindo-se $\sigma^{2}$ pela estimativa $s^{2}$, resul

tou:

$$
\hat{\mathrm{V}}(\hat{\mathrm{c}})=\frac{5}{16} \mathrm{~s}^{2} \quad \text {. }
$$

Esse resultado é o mesmo quando se deduz a fórmula da variância do contraste entre duas médias a par tir da expressão do cálculo de parcela perdida (LUCAS,1956) ou pelo Método Direto apresentado por GODOI (1971) conhecendo-se as expressões de $\hat{t}_{1}$ e $\hat{t}_{2}$.

o autor apresentou ainda um exemplo numérico para ilustrar as deduções feitas e também a introdução do efeito de blocos no modelo completo sem entrar em dedu- 
ções teóricas.

Apresenta, ademais, um programa para computa dor eletrônico na linguagem FORTRAN-1130, e sugere um modelo matemático para a análise de covariância de dados de delineamentos em "switchback".

KALIL (1971) apresentou as fórmulas obtidas por LUCAS (1956) para anālise de variância nos ensaios de reversão. O autor apresentou como exemplo, ensaios utilizando as fórmulas propostas considerando também, casos de parcela perdida.

Lucas (1975), segundo GODOI (1983), propôs o estudo dos.componentes de variância quando a interação pe ríodo (linear) $x$ animais é considerada aleatória, linha de pesquisa não seguida pelo autor.

GODOI (1983), com o objetivo de implementar a análise de dados de produção de leite, propôs o estudo de dois modelos. No Modelo I o autor admitiu a independên cia dos erros e no Modelo II foi admitida a hipótese de erros correlacionados segundo o esquema auto-regressivo.

O autor chegou à conclusão de que os dados de produção de leite tomados para ensaios em "switchback" possuem correlações entre períodos do tipo auto-regressivo. Portanto, ao comparar os dois modelos o autor concluiu que o Modelo II conduz a testes de significância mais poderosos. 
43.

3. MATERIAL

A exemplificação da metodologia aqui apresen tada será feita com os dados obtidos da pesquisa "Utiliza ção de Sub-Produtos da Fermentação Alcoólica na Alimenta ção de Vacas em Lactação", realizada no Estábulo Experimental do Departamento de Zootecnia da Escola Superior de Agricultura "Luiz de Queiroz", no período de junho a dezembro de 1982.

0 esquema adotado para se testar três tratamentos, foi o seguinte: 


\begin{tabular}{|c|ccc|}
\hline \multirow{2}{*}{ ANIMAL } & I P E R I O D O S \\
\hline BLOCO 1 & & & \\
1 & A & B & A \\
2 & A & C & A \\
3 & B & A & B \\
4 & B & C & B \\
5 & C & A & C \\
6 & C & B & C \\
\hline BLOCO 2 & & & \\
7 & A & B & A \\
8 & A & C & A \\
9 & B & A & B \\
10 & B & C & B \\
11 & C & A & C \\
12 & C & B & C \\
\hline
\end{tabular}

e os dados obtidos para produção de leite, em quilogramas $(\mathrm{kg})$, constam da Tabela 1 . 
Tabela 1 - Produção de leite (kg) de vacas recebendo dife rentes fontes de nitrogênio.

\begin{tabular}{|c|c|c|c|c|c|c|c|}
\hline \multicolumn{2}{|c|}{ ANIMAIS } & \multicolumn{6}{|c|}{$P E R \underset{I I}{I_{I}} O D O S$} \\
\hline \multirow{6}{*}{$\begin{array}{l}-1 \\
0 \\
0 \\
0 \\
0 \\
0\end{array}$} & 1 & 20,04 & (A) & 21,19 & (B) & 17,52 & $(A)$ \\
\hline & 2 & 17,30 & (A) & 19,01 & (C) & 15,07 & (A) \\
\hline & 3 & 25,36 & (B) & 26,39 & (A) & 22,39 & (B) \\
\hline & 4 & 15,52 & (B) & 13,53 & (C) & 11,57 & (B) \\
\hline & 5 & 19,93 & (C) & 17,92 & (A) & 13,93 & (C) \\
\hline & 6 & 13,12 & (C) & 12,18 & (B) & 12,82 & (C) \\
\hline \multirow{6}{*}{ 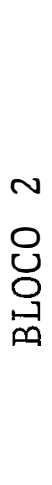 } & 7 & 14,08 & (A) & 13,80 & (B) & 11,74 & (A) \\
\hline & 8 & 19,04 & (A) & 18,65 & (C) & 15,81 & (A) \\
\hline & 9 & 16,19 & (B) & 14,94 & (A) & 13,13 & (B) \\
\hline & 10 & 25,07 & (B) & 24,15 & (C) & 19,92 & (B) \\
\hline & 11 & 18,95 & (C) & 17,64 & (A) & 14,02 & (C) \\
\hline & 12 & 18,75 & (C) & 17,86 & (B) & 13,41 & (C) \\
\hline
\end{tabular}


46.

4. DESENVOLVIMENTO TEORICO

4.1 - Introdução

Para o desenvolvimento teórico, foi suposto um ensaio em "switchback" com p tratamentos, $\underline{v}$ animais, J períodos, $S=p \cdot(p-1)$ sequências e $L=(p-1)$ blocos, conforme LUCAS (1956).

Como em um ensaio em "switchback" as generali zações tornam-se muitas vezes difíceis e pouco esclarecedo ras, houvemos por bem construir um exemplo inicial e usá- lo como suporte, sem perda de generalidade, para as deduções.

Com esse objetivo foi adotado o seguinte esquema: 
47.

\begin{tabular}{|c|ccc|}
\hline \multirow{2}{*}{ ANIMAIS } & P E R I O D O S \\
\hline BLOCO 1 & I & I I & I I I \\
1 & & & \\
2 & A & B & A \\
3 & B & C & B \\
BLOCO 2 & C & A & C \\
4 & & & \\
5 & B & A & B \\
6 & C & B & C \\
\hline
\end{tabular}

As características desse esquema representa tivo descrito acima são:

$$
\begin{aligned}
\mathrm{p} & =3=\text { nümero de tratamentos; } \\
\mathrm{J} & =3=\text { nümero de períodos; } \\
\mathrm{L} & =(\mathrm{p}-1)=2 \text { = nümero de blocos, onde cada bloco } \\
& \text { possui } \mathrm{p} \text { sequências; } \\
\mathrm{v}= & 6=\text { número de animais; } \\
\mathrm{S} & =\mathrm{p}(\mathrm{p}-1)=6 \text { = nümero de sequências. }
\end{aligned}
$$


Seja o modelo Iinear $y=X \beta+\varepsilon$, onde:

$y$ é um vetor de realizações de variáveis aleatórias; $X$ é uma matriz conhecida, dos coeficientes dos parâmetros;

$\beta$ é um vetor de parâmetros desconhecidos;

$\varepsilon$ é um vetor de erros aleatórios, tal que

$$
\varepsilon \cap N\left(\phi, I \sigma^{2}\right) ;
$$

caracterizado por:

$$
\begin{aligned}
y_{i j k \ell}=m & +b_{\ell} z_{j}+v_{i}+t_{k}+\alpha_{1} x_{j}+\alpha_{2} z_{j}+c_{i} x_{j}+ \\
& +e_{i j k \ell}
\end{aligned}
$$

onde:

$$
\begin{aligned}
& y_{i j k \ell} \text { é o valor observado na i-ésima vaca, do j-és } \underline{i} \\
& \text { mo período, submetida ao k-ésimo tratamento do } \\
& \quad \ell \text {-ésimo bloco; } \\
& m \text { é a média geral; } \\
& b_{\ell} \text { é o efeito do bloco } \ell \quad(\ell=1,2, \ldots, L) ; \\
& v_{i} \text { é o efeito da vaca } \underline{i}(i=1,2, \ldots, v) ; \\
& t_{k} \text { é o efeito do tratamento } k(k=1,2, \ldots, p) ; \\
& \alpha_{1} \text { é o efeito linear de período; } \\
& \alpha_{2} \text { é o efeito quadrático de período; } \\
& \left.c_{i} \text { é o efeito da interação período (linear }\right) x \text { va- } \\
& \text { cas ; }
\end{aligned}
$$




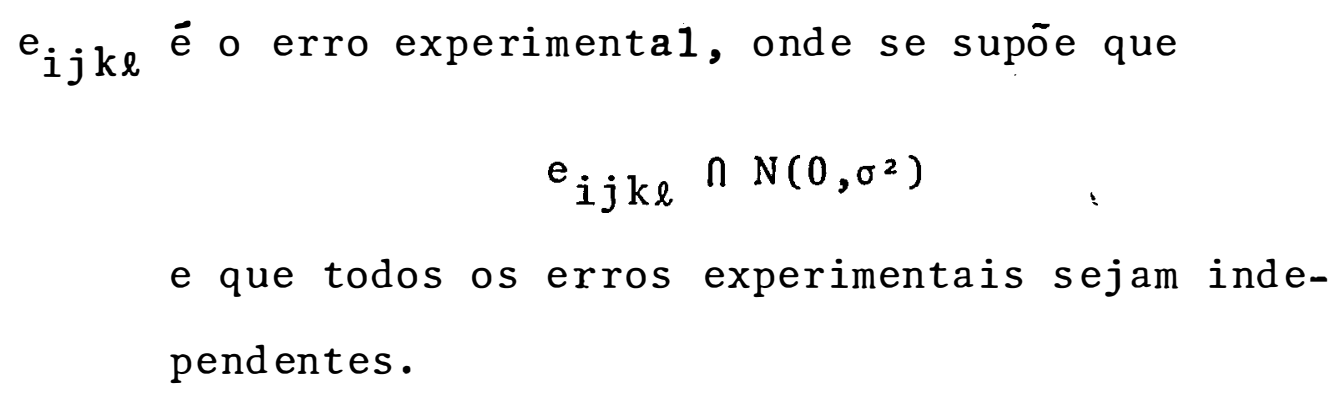

Além disso,

$$
\begin{aligned}
& x_{j}=\left\{\begin{array}{rrrr}
-1 & \text { se } & j=1 & (19 \text { Período }) \\
0 & \text { se } j=2 & (29 \text { Período }) \\
1 & \text { se } & j=3 & (39 \text { Período })
\end{array}\right. \\
& z_{j}=\left\{\begin{array}{rrrr}
1 & \text { se } & j=1 & (19 \text { Período }) \\
-2 & \text { se } & j=2 & (29 \text { Período }) \\
1 & \text { se } & j=3 & (39 \text { Período })
\end{array}\right.
\end{aligned}
$$

que correspondem à aplicação do conceito de polinômios ortogonais para o desmembramento dos componentes linear e qua drático dos períodos.

4.2.1 - Exigências do modelo adotado

Conforme sugerido na descrição do modelo, sua adequação está restrita a certas exigências, conforme rela cionado a seguir: 
i) Aditividade dos efeitos;

ii) Erros com distribuição normal, onde $E\left(e_{i}\right)=0$ e $\mathrm{V}\left(\mathrm{e}_{\mathrm{i}}\right)=\sigma^{2} ;$

iii) Homogeneidade de variâncias;

iv) Independência dos erros.

Para que se possa analisar um conjunto de da dos, através do modelo proposto neste estudo, torna-se ne cessária a aplicação de testes que permitam verificar se as pressuposições do modelo não estão sendo violadas em prejuízo da confiabilidade dos testes $F$ na análise de variância .

Dentre os processos mais comuns utilizados pa ra esse fim, daremos prioridade, neste estudo, à análise gräfica de resíduos, conforme procedimentos amplamentedés critos em ANSCOMBE (1961), ANSCOMBE e TUKEY (1963), NETER e WASSERMAN (1977), dentre outros.

\section{3 - Equações Normais}

Para a obtenção do sistema de equações normais $X^{\prime} X \hat{\beta}=X^{\prime} y$, efetuamos a partição da matriz $X$, conforme CHAKRABARTI (1962), PIMENTEL GOMES (1967), IEMMA (1981), entre outros. 
Então, a matriz relativa ao exemplo citado em (4.1) resultou:

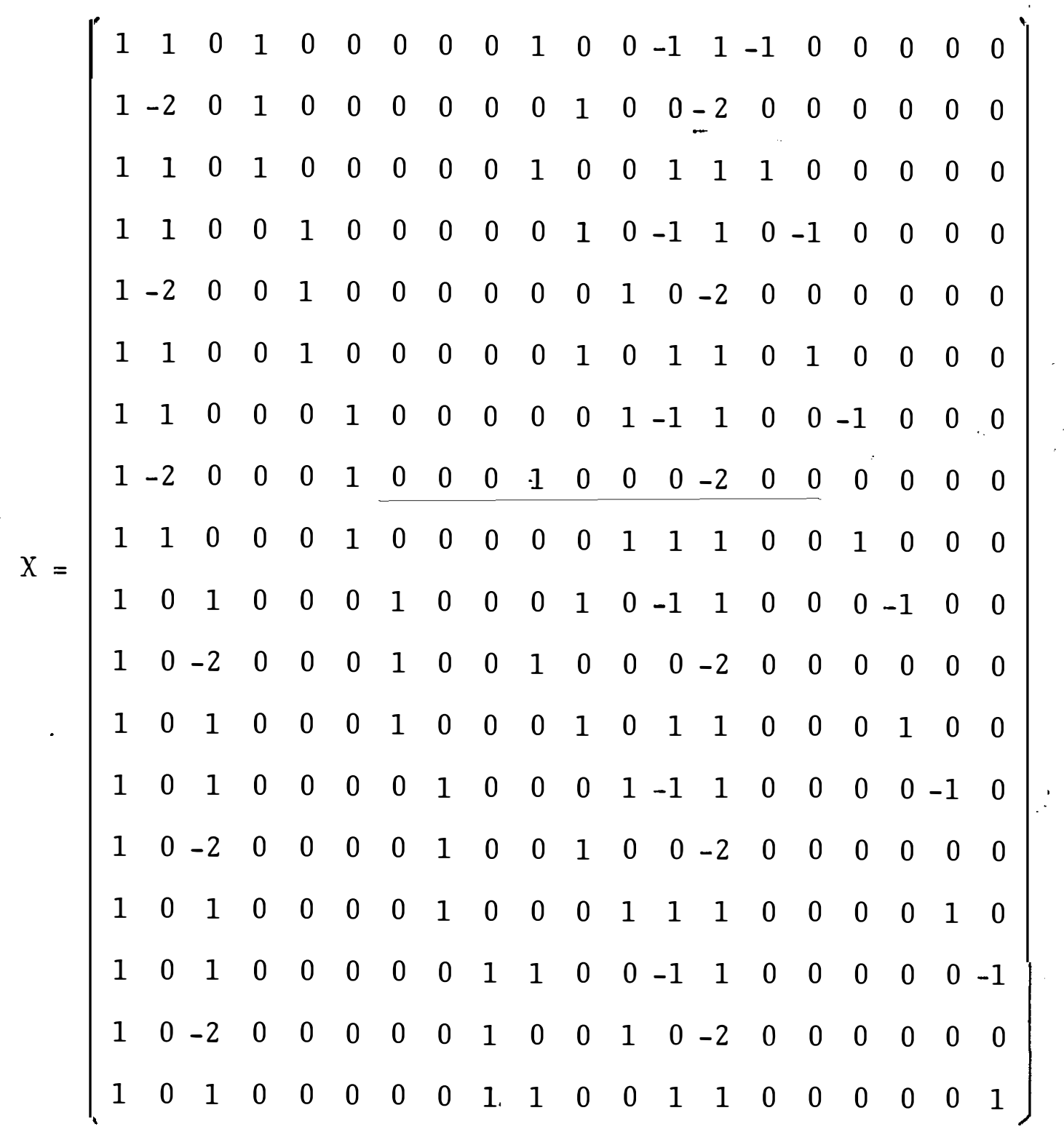

de dimensões Nx(L $+v+p+(J-1)+v+1)$, ou

$$
\mathrm{x}=\left[\begin{array}{l:l:l:l:l:l}
\mathrm{x}_{1} & \mathrm{x}_{2} & \mathrm{x}_{3} & \mathrm{x}_{4} & \mathrm{x}_{5} & \mathrm{x}_{6}
\end{array}\right]
$$


onde:

$$
\begin{aligned}
& x_{1}=\text { vetor dos coeficientes da média, de dimensões } \\
& \mathrm{X}_{2}=\text { matriz dos coeficientes associados aos blocos, } \\
& \text { de dimensões (N) x (L); } \\
& \mathrm{x}_{3}=\text { matriz dos coeficientes associados a vacas, de } \\
& \text { dimensões }(N) \times(v) \text {; } \\
& \mathrm{X}_{4}^{\prime}=\text { matriz dos coeficientes associados aos trata- } \\
& \text { mentos, de dimensões (N) } x(p) \text {; } \\
& X_{5}=\text { matriz dos coeficientes associados aos efeitos } \\
& \text { linear e quadrático de períodos, de dimensões } \\
& \text { (N) } x(J-1) \text {; } \\
& X_{6}=\text { matriz dos coeficientes associados aos efeitos } \\
& \text { da interação período (linear) x vacas, de di- } \\
& \text { mensões }(N) \times(v) \text {. }
\end{aligned}
$$

Assim, o Sistema de Equações Normais obtido,

foi : 


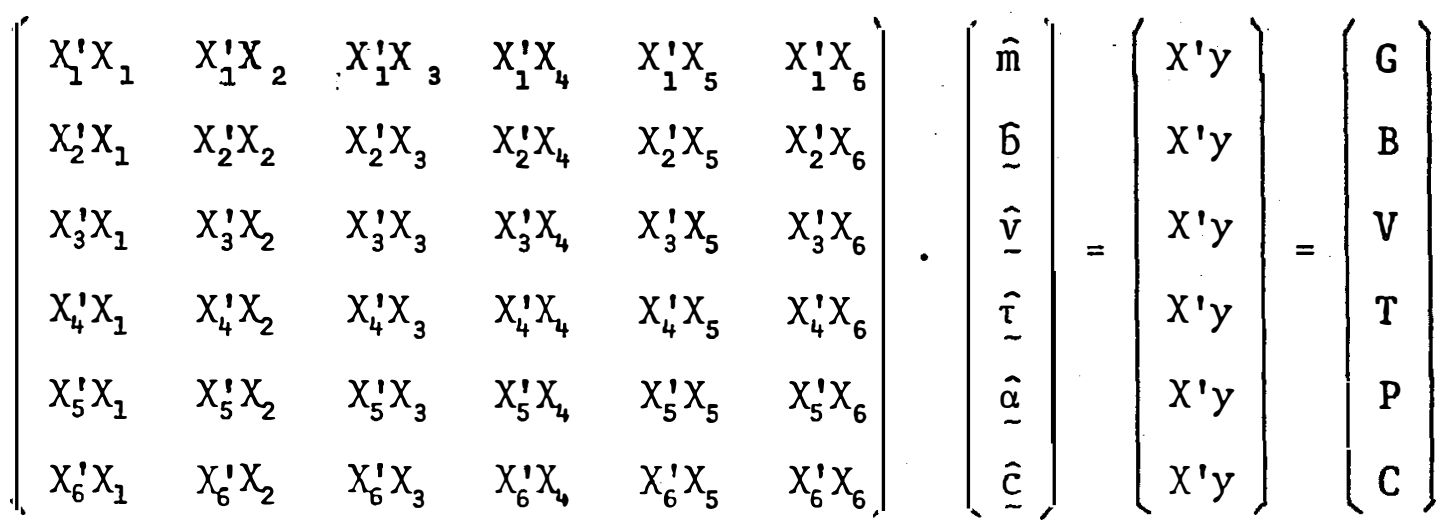

onde:

$$
\begin{aligned}
& X^{\prime} X \text { tem dimensões }[L+v+p+(J-1)+v+1] \text {; } \\
& X_{1}^{\prime} X_{1}=M_{1}=N=\text { número de observações, que no exemplo } \\
& \text { em questão, resultou } X_{1}^{\prime} X_{1}=M_{1}=18 \text {; }
\end{aligned}
$$

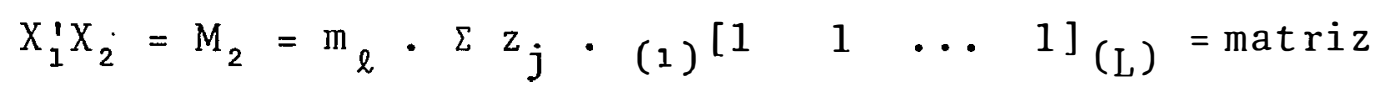

$$
\begin{aligned}
& \text { associada à soma, por animal, dos coeficientes } \\
& \text { dos efeitos de blocos, sendo } m_{\ell} \text { o nümero de a- } \\
& \text { nimais do bloco } \underline{\ell} \text {, e } \Sigma z_{j}=0 \text {, então: } \\
& X_{1}^{\prime} X_{2}=M_{2}=(1)\left[\begin{array}{llll}
0 & 0 & \ldots & 0
\end{array}\right]_{(\mathrm{L})}=(1)^{\phi}(\mathrm{L}) \text {, } \\
& \text { onde }(1)^{\phi}(\underbrace{}_{1}) \bar{e} \text { uma matriz nula. Assim, no exem- } \\
& \text { plo obteve-se } X_{1}^{1} X_{2}=M_{2}=(1)^{\phi}(2) \text {; } \\
& X_{1}^{\prime} X_{3}=M_{3}=(1)\left[\begin{array}{llll}
J & J & J
\end{array}\right]_{(v)}=\text { matriz associada } \\
& \text { ao número de períodos, onde } \underline{v} \text { é o número de } \\
& \text { animais. Então: } X_{1}^{\prime} X_{3}=M_{3}=3(1) E_{(6)} \text {, e E } \\
& \text { uma matriz cujos elementos são todos iguais à } \\
& \text { unidade; }
\end{aligned}
$$




$$
\begin{aligned}
& \left.X_{1}^{\prime} X_{4}=M_{4}=(1)^{\left[r_{k}\right.} \quad r_{k} \quad \ldots \quad r_{k}\right]_{(p)}=\text { vetor associa } \\
& \text { do ao nümero de repetições de tratamentos, is } \\
& \text { to é, ao nümero de vezes em que cada tratamen } \\
& \text { to foi aplicado durante o ensaio. No exemplo } \\
& \text { citado em (4.1) o nümero de repetições } r_{k} \text { é } \\
& \text { igual a } 6 \text {, pois cada tratamento foi utiliza- } \\
& \text { do } 6 \text { vezes durante o experimento, portanto: } \\
& X_{1}^{\prime} X_{4}=M_{4}=6 \text { (1) } E_{(3)} \text {; }
\end{aligned}
$$

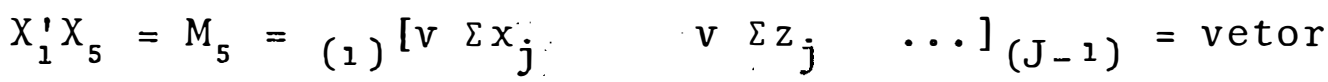

$$
\begin{aligned}
& \text { associado à soma dos coeficientes linear e qua } \\
& \text { drático de períodos. Sendo } \Sigma x_{j}=\Sigma z_{j}=0 \text {, en- } \\
& \text { tão: } X_{1}^{\prime} X_{5}=M_{5}=(1)\left[\begin{array}{llll}
0 & 0 & \ldots . .
\end{array}\right]_{(J-1)}={ }_{(1)^{\phi}(J-1)} \cdot \\
& \text { Como no exemplo em estudo } \mathrm{J}=3 \text {, então: } \\
& X_{1}^{\prime} X_{5}=M_{5}=(1)^{\phi}(2) ; \\
& X_{1}^{\prime} X_{6}=M_{6}=(1)\left[\Sigma x_{j} \quad \Sigma x_{j} \quad \ldots \quad \sum x_{j}\right](v)=\text { vetor as } \\
& \text { sociado à soma dos coeficientes da Interasão } \\
& \text { Período (linear) } x \text { Vacas. Sendo } v=6 \text {, obtive } \\
& \operatorname{mos} X_{1}^{\prime} X_{6}=M_{6}=(1)^{\phi}(6) \text {; }
\end{aligned}
$$

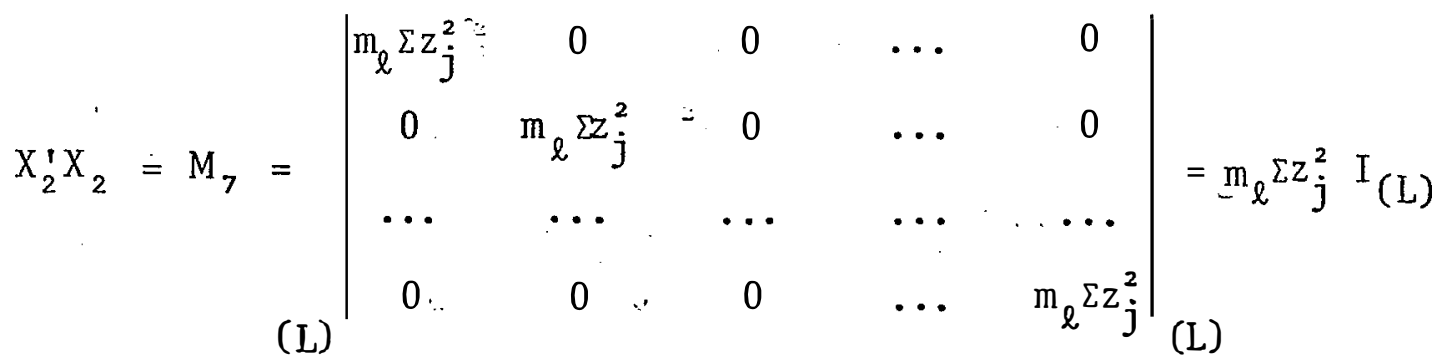


onde $I_{(L)}$ é uma matriz identidade; de dimensão (L), $\underline{m}_{\ell}$ é o número de animais no bloco $\ell$. Temos no exemplo $m_{\ell}=3, \Sigma z_{j}^{2}=6$ e $L=2$, logo: $X_{2}^{\prime} X_{2}=M_{7}=3 \cdot 6 I_{(2)}$;

$\mathrm{X}_{2}^{\prime} \mathrm{X}_{3}=\mathrm{M}_{8}=\left[\begin{array}{ccccccc}\Sigma z_{j} & \Sigma z_{j} & \Sigma z_{j} & \ldots & 0 & 0 & 0 \\ \ldots & \ldots & \ldots & \ldots & \ldots & \ldots & \ldots \\ 0 & 0 & 0 & \ldots & \Sigma z_{j} & \Sigma z_{j} & \Sigma z_{j}\end{array}\right]_{(v)}=(L)^{\phi}(v)$ è a matriz: nula associada à soma dos coeficien tes quadráticos de blocos por animal. Como temos 2 blocos e 6 animais, a matriz ficou: $X_{2}^{\prime} X_{3}=M_{8}=(2)^{\phi}(6) ;$

$X_{2}^{\prime} X_{4}=M_{9}=\left[\begin{array}{ccccc}\Sigma z_{j} & \Sigma z_{j} & \cdots & \Sigma z_{j} & \Sigma z_{j} \\ \Sigma z_{j} & \Sigma z_{j} & \cdots & \Sigma z_{j} & \Sigma z_{j} \\ \ldots & \ldots & \cdots & \cdots & \cdots \\ \Sigma z_{j} & \Sigma z_{j} & \cdots & \Sigma z_{j} & \Sigma z_{j}\end{array}\right]=(L)^{\phi}(P)$ é a matriz nula associada à soma dos coeficien tes quadráticos de blocos, por tratamento, resultando, para o caso de 2 blocos e 3 tratamen tos: $X_{2}^{\prime} X_{4}=M_{9}=(2)_{(3)}^{\phi}$;

$$
X_{2}^{\prime} X_{5}=M_{10}=m_{\ell}\left[\begin{array}{ll}
\Sigma x_{j}{ }^{2} j & \Sigma z_{j}^{2} \\
\Sigma x_{j}{ }^{2} j & \Sigma z_{j}^{2}
\end{array}\right)_{(J-1)}
$$


é a matriz associada ao produto dos coeficien tes quadráticos de blocos pelos coeficientes linear e quadrático de períodos. Como $\Sigma x_{j} \cdot z_{j}=0$, $\Sigma z_{j}^{2}=6$, e no nosso exemplo com $m_{\ell}=3$, obtive mos

$X_{2}^{\prime} X_{5}=M_{10}=3\left(\begin{array}{ll}0 & 6 \\ 0 & 6\end{array}\right)_{(2)} ;$

$X_{2}^{\prime} X_{6}=M_{11}=(L)^{\phi}(v)$, $\vec{e}$ a matriz nula associada ao pro duto dos coeficientes quadráticos de blocos pe los coeficientes lineares da interação Péríodo (linear) $x$ Vacas. Para o exemplo em questão: $X_{2}^{\prime} X_{6}=M_{11}=\left({ }_{2}\right)^{\phi}(6)$;

$X_{3}^{\prime} X_{3}=M_{12}=J_{-} I_{(v)}$, é a matriz diagonal associada ao número de períodos. Como temos $\mathrm{J}=3$ períodos, obtivemos: $X_{3}^{\prime} X_{3}=M_{12}=3 I_{(6)}$;

$X_{3}^{\prime} X_{4}=M_{13}=\left[\begin{array}{cccc}a_{11} & a_{12} & \ldots & a_{1 p} \\ a_{21} & a_{22} & \ldots & a_{2 p} \\ \ldots & \ldots & \ldots & \ldots \\ a_{v} & a_{v 2} & \ldots & a_{v p}\end{array}\right]_{(p)}$

é a matriz associada ao número de vezes em que cada animal recebe o tratamento k durante o en saio. No exemplo citado em (4.1), temos o ani- 
mal 1 na sequência: $A B A, \operatorname{logo}_{11}$ que será 0 número de vezes que o animal 1 recebe o trata mento $A$, isto é, tratamento 1 , é igual a 2 , e $a_{12}$ que é o número de vezes que o animal 1 re cebe o tratamento 2 (B), é igual a 1 , e $a_{13}$ o número de vezes que o animal 1 recebe o tra tamento 3 (C) é igual a zero. Portanto:

$X_{3}^{\prime} X_{4}=M_{13}=\left[\begin{array}{lll}2 & 1 & 0 \\ 0 & 2 & 1 \\ 1 & 0 & 2 \\ 1 & 2 & 0 \\ 0 & 1 & 2 \\ 2 & 0 & 1\end{array}\right]_{(3)}$

$\mathrm{X}_{3}^{\prime} \mathrm{X}_{5}=\mathrm{M}_{14}=(\mathrm{v})^{\phi}\left(\mathrm{J}_{-1}\right)$, é a matriz associada à soma por animal dos coeficientes linear e quadráti co dos períodos. Logo: $X_{3}^{1} X_{5}=M_{14}=(6)^{\phi}(2)$;

$X_{3}^{\prime} X_{6}=M_{1 \dot{5}}=\Sigma X_{j} \cdot I(v)=(v)^{\phi}(v)$, é a matriz associada à soma por animal dos coeficientes da Interação Períodos (linear) x Vacas. Para o exemplo em estudo, obtivemos:

$X_{3}^{\prime} X_{6}=M_{15}=(6)^{\phi}(6)$; 


$$
\begin{aligned}
X_{4}^{\prime} X_{4}= & M_{16}=r_{k} \cdot I_{(p)} \text {, é a matriz diagonal asso- } \\
& \text { ciada ao número de repetiçōes dos tratamen- } \\
& \text { tos. Como no exemplo em estudo cada tratamen } \\
& \text { to aparece } 6 \text { vezes durante o ensaio, temos } \\
& r_{k}=6 . \text { Portanto: } X_{4}^{\prime} X_{4}=M_{16}=6 \cdot I_{(3)} ;
\end{aligned}
$$

$\mathrm{X}_{4}^{\prime} \mathrm{X}_{5}=\mathrm{M}_{17}=(\mathrm{p})^{\phi}(\mathrm{J}-1)$, é a matriz resultante do produto da matriz dos coeficientes dos efeitos de tratamentos pela matriz dos coeficien tes linear e quadrático dos períodos. Para o exemplo citado, obtivemos $X_{4}^{\prime} X_{5}=M_{17}=\underset{(3)(2)}{\phi}$; $X_{4}^{\prime} X_{6}=M_{18}=(p)^{\phi}(v)$, é a matriz resultante do pro duto dos coeficientes dos efeitos de tratamentos pela matriz dos coeficientes da Interação Período (1inear) x Vacas. Então:

$$
\begin{gathered}
X_{4}^{\prime} X_{6}=M_{18}=(3)^{\phi}(6) ; \\
X_{5}^{\prime} X_{5}=M_{19}=V \cdot\left(\begin{array}{ccc}
\sum X_{j}^{2} & \cdots & 0 \\
0 & \cdots & \sum z_{j}^{2}
\end{array}\right)_{(J-1)}
\end{gathered}
$$

è a matriz diagonal associada ao produto do número de animais pela soma dos quadrados dos coeficientes linear e quadrático de períodos, que para o exemplo em estudo, resultou:

$$
X_{5}^{\prime} X_{5}=M_{19}=6 \cdot\left[\begin{array}{ll}
2 & 0 \\
0 & 6
\end{array}\right]_{(2)}=\left(\begin{array}{rr}
12 & 0 \\
0 & 36
\end{array}\right)_{(2)} \text {; }
$$


59.

$$
X_{5}^{\prime} X_{6}=M_{20}=\left(\begin{array}{cccc}
\Sigma x_{j}^{2} & \sum x_{j}^{2} & \cdots & \Sigma x_{j}^{2} \\
\Sigma x_{j} \sum z_{j} & \Sigma x_{j} \sum z_{j} & \cdots & \sum x_{j} \sum z_{j}
\end{array}\right)_{(v)}
$$

é a matriz associada ao produto dos coeficien tes linear e quadrático de períodos pelos coe ficientes da Interação Períodos (1inear) x Va cas, resultando, para o exemplo em estudo:

$$
X_{5}^{1} X_{6}=M_{20}=\left(\begin{array}{llll}
2 & 2 & \ldots & 2 \\
0 & 0 & \cdots & 0
\end{array}\right)_{(6)} \text {; }
$$

$\mathrm{X}_{6}^{1} \mathrm{X}_{6}=\mathrm{M}_{21}=\sum \mathrm{X}_{\mathrm{j}}^{2} \mathrm{I}_{(\mathrm{v})}$, é a matriz diagonal associada à soma dos quadrados dos coeficientes da Interação Período (linear) $x$ Vacas. Como $\Sigma x_{j}^{2}=$ $=2$ e no exemplo citado em 4.1 temos 6 animais, resultou: $X_{6}^{1} X_{6}=M_{21}=2 \cdot I_{(6)}$.

De modo análogo, o vetor $\hat{\beta}$ ficou constituído

de :

$$
\begin{aligned}
& \hat{\mathrm{m}}=\text { média geral; } \\
& \underline{\underline{b}}=\text { vetor das soluções de mínimos quadrados para efe } \underline{i} \\
& \text { tos de blocos; } \\
& \underline{\underline{v}}=\text { vetor das soluções de mínimos quadrados para efe } \underline{i} \\
& \text { tos de vacas; } \\
& \bar{\tau}=\text { vetor das soluções de mínimos quadrados para efe } \underline{i} \\
& \text { tos de tratamentos; }
\end{aligned}
$$


$\widehat{\alpha}=$ vetor das soluções de mínimos quadrados para efeitos linear e quadrätico de períodos; $\hat{\mathbf{c}}=$ vetor das soluções de mínimos quadrados para efeitos da interação Período (linear) $x$ Vacas.

Para o vetor X'y, tem-se:

$X_{1}^{\prime} y=G=$ total das observaçōes $y_{i j k \ell} ;$
$X_{2}^{\prime} y=B=\left(\begin{array}{c}B_{1} \\ B_{2}\end{array}\right)$, onde: $\mathrm{B}_{\ell}=\Sigma \mathrm{D}_{i}=$ soma dos $\mathrm{D}_{\mathbf{i}}$ dos animais que pertencem ao bloco $\underline{\ell}$; e $D_{i}=y_{i 1 k \ell}-2 y_{i 2 k \ell}+y_{i 3 k \ell}$, segundo BRANDT (1938) e LUCAS (1956);

$\mathrm{X}_{3}^{1} \mathrm{y}=\mathrm{V}=$ vetor dos totais de vacas;

$\mathrm{X}_{4}^{\prime} \mathrm{Y}=\mathrm{T}=$ vetor dos totais de tratamentos;

$X_{5}^{\prime} y=P=\left(\begin{array}{l}P_{3}-P_{1} \\ P_{1}-2 P_{2}+P_{3}\end{array}\right)$,

onde: $\mathrm{P}_{1}=$ total do 19 período; $\mathrm{P}_{2}=$ total do 29 período; $\mathrm{P}_{3}=$ total do 3 e período. 


$$
x_{6}^{\prime} y=C_{l}=\left(\begin{array}{c}
y_{13 k \ell}-y_{11 k \ell} \\
\ldots \ldots \ldots \ldots \ldots \\
\ldots \ldots \ldots \ldots . \\
y_{v_{3 k \ell}}-y_{v_{1 k}}
\end{array}\right) ;
$$

resultando em:

$$
\left[\begin{array}{llllll}
M_{1} & \phi & M_{3} & M_{4} & \phi & \phi \\
\phi & M_{7} & \phi & \phi & M_{-10} & \phi \\
M_{3}^{\prime} & \phi & M_{12} & M_{13} & \phi & \phi \\
M_{4}^{\prime} & \phi & M_{13}^{\prime} & M_{16} & \phi & \phi \\
\phi & M_{10}^{\prime} & \phi & \phi & M_{19} & M_{20} \\
\phi & \phi & \phi ! & \phi & M_{20}^{\prime} & M_{21}
\end{array}\right) \cdot\left(\begin{array}{c}
\hat{m} \\
\tilde{~} \\
\tilde{v} \\
\tilde{v} \\
\tilde{\tau} \\
\tilde{\alpha} \\
\tilde{c} \\
\tilde{c}
\end{array}\right)=\left(\begin{array}{c}
G \\
B \\
V \\
V \\
T \\
P \\
C
\end{array}\right)
$$

Por outro lado, sabemos, da teoria geral,que o sistema de equações normais é sempre consistente, admitindo mais de uma solução nos casos como este, em que a matriz X não é de posto completo.

Desse modo, visando à obtenção de solução única para o sistema, adotamos o uso de restrições não estimáveis nas soluções, conforme amplamente abordado em textos de Estatística Experimental e Modelos Lineares. Es se procedimento pareceu ser adequado, pois, o uso das in- 
versas generalizadas, embora'se constitua num procedimen to elegante, é em geral inviāvel para pesquisadores que não dispõem de computadores de grande porte, adequados ao: uso de "pacotes".

Assim, na obtenção de solução ünica, foram tomadas as restrições:

$$
\begin{aligned}
& \underset{i=1}{v} \hat{v}_{i}=0 ; \sum_{\ell=1}^{L} b_{\ell}=0 ; \sum_{k=1}^{p} \hat{t}_{k}=0 ; \\
& \sum_{i=1}^{v} \hat{c}_{i}=0
\end{aligned}
$$

Nesse contexto, ressalta-se aqui, que alguns produtos de interesse, que aparecem em (II), podem ser colocados sob a forma das restrições citadas, e este fato simplificará sobremaneira a solução do sistema de equações normais. São eles:

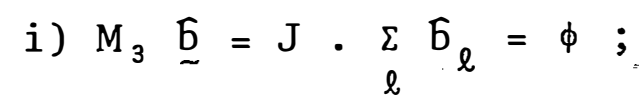

ii) $M_{3} \underset{\sim}{\hat{v}}=J \cdot \sum_{i} \hat{v}_{i}=\phi ;$

iii) $M_{4} \hat{\tau}=r_{k} \cdot \underset{k}{\sum} \hat{t}_{k}=\phi$;

iv) $M_{10}^{1} \underset{:}{b}=m_{\ell} \cdot \Sigma z_{j}^{2} \cdot \sum_{\ell} b_{\ell}=\phi ;$

v) $M_{20} \underset{\sim}{\hat{c}}=\sum x_{j}^{2} \sum_{i}^{\sum} \hat{c}_{i}=\phi$. 
Consideradas as restrições, o sistema de equa ções normais (II), resultou:

$$
\begin{aligned}
& M_{1} \hat{m} \\
& =\mathrm{G} \\
& M_{7} \underset{\sim}{\sim} \\
& +M_{10} \hat{\alpha} \\
& =\mathrm{B} \\
& M_{3}^{\prime} \hat{m} \\
& +M_{12} \hat{\sim}+M_{13} \hat{I} \\
& =\mathrm{V} \\
& M_{4}^{\prime} \hat{m} \\
& +M_{13}^{\prime} \underset{\sim}{\hat{v}}+M_{16} \underset{\sim}{\hat{\tau}} \\
& =\mathrm{T} \\
& M_{19} \stackrel{\alpha}{\sim} \\
& =\mathrm{P} \\
& M_{20}^{\prime} \underset{\sim}{\hat{\alpha}}+M_{21} \underline{\hat{c}}=C \\
& \text { SUB-SISTEMA } 1 \text { : } \\
& M_{1} \hat{\mathrm{m}} \quad=\mathrm{G} \\
& M_{3}^{\prime} \hat{m}+M_{12} \underset{\sim}{\hat{v}}+M_{3} \quad \hat{\tau}=V \\
& M_{4}^{\prime} \hat{\mathrm{m}}+M_{13}^{\prime} \underset{\tilde{v}}{\hat{v}}+M_{16} \underset{\sim}{\hat{I}}=\mathrm{T}
\end{aligned}
$$


64.

\section{SUB-SI STEMA 2}

$$
\begin{aligned}
M_{7} \underset{\sim}{\underline{b}}+M_{10} \stackrel{\hat{\alpha}}{\sim}=B \\
M_{19} \stackrel{\hat{\alpha}}{\sim}=P \\
M_{20}^{\prime} \underset{\sim}{\hat{\alpha}}+M_{21} \underset{\sim}{\hat{c}}=C
\end{aligned}
$$

4.3.1 - Solução do sistema para a média

$$
\text { Através do sub-sistema } 1 \text {, }
$$

$$
M_{1} \hat{m}=G \text {, }
$$

obtivemos:

$$
\begin{gathered}
\hat{m}=M_{1}^{-1} G . \\
\text { Como } M_{1}=N \text {, escalar, a solução do sistema pa }
\end{gathered}
$$

ra $\hat{\mathrm{m}}$ é:

$$
\hat{\mathrm{m}}=\frac{\mathrm{G}}{\mathrm{N}}
$$

e, no exemplo em questão:

$$
\hat{\mathrm{m}}=\frac{\mathrm{G}}{18} \text {. }
$$




$$
\begin{array}{r}
\text { 4.3.2 - Solução do sistema para efeitos de trata } \\
\text { mentos eliminando-se efeitos de vacas }
\end{array}
$$

Para eliminar os efeitos de vacas, optamos conforme CHAKRABARTI (1962), por pré-multiplicar ambos os membros do sistema II pela matriz W definida como:

$$
\begin{aligned}
& W=\left[\begin{array}{llllll}
\phi & \phi & -M_{13}^{1} M_{12}^{-1} & I & \phi & \phi
\end{array}\right] . \\
& \text { Então, efetuando o produto: }
\end{aligned}
$$

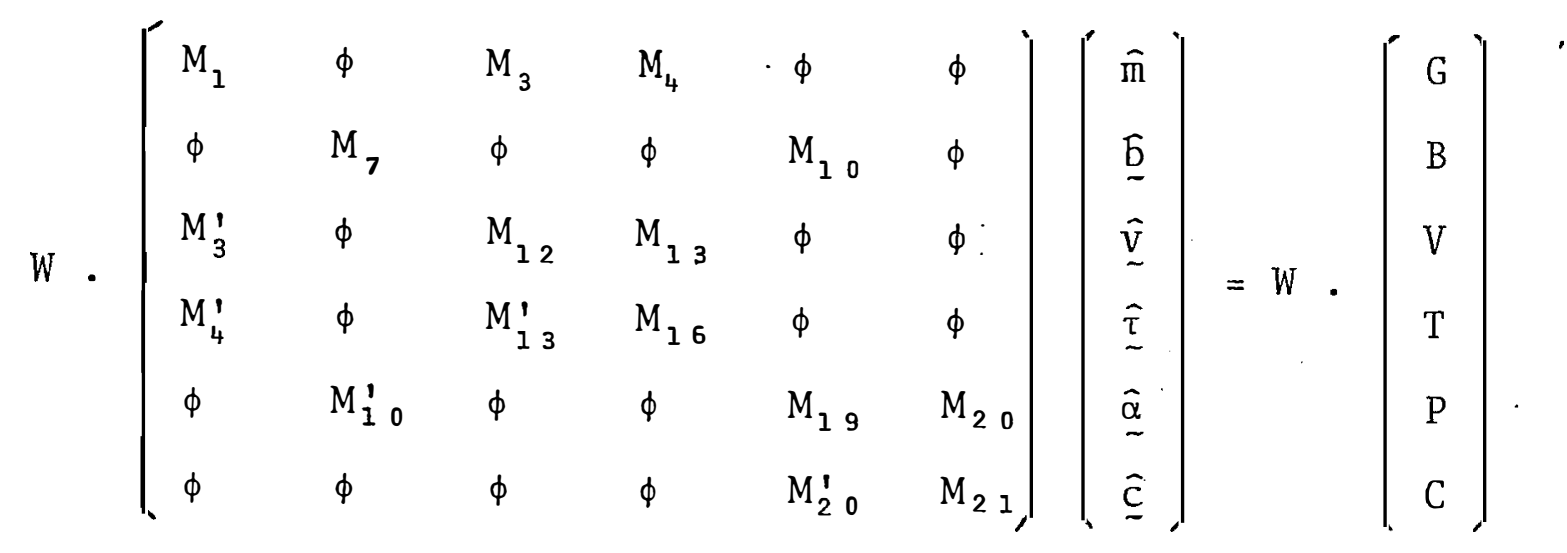

obtivemos: 
66.

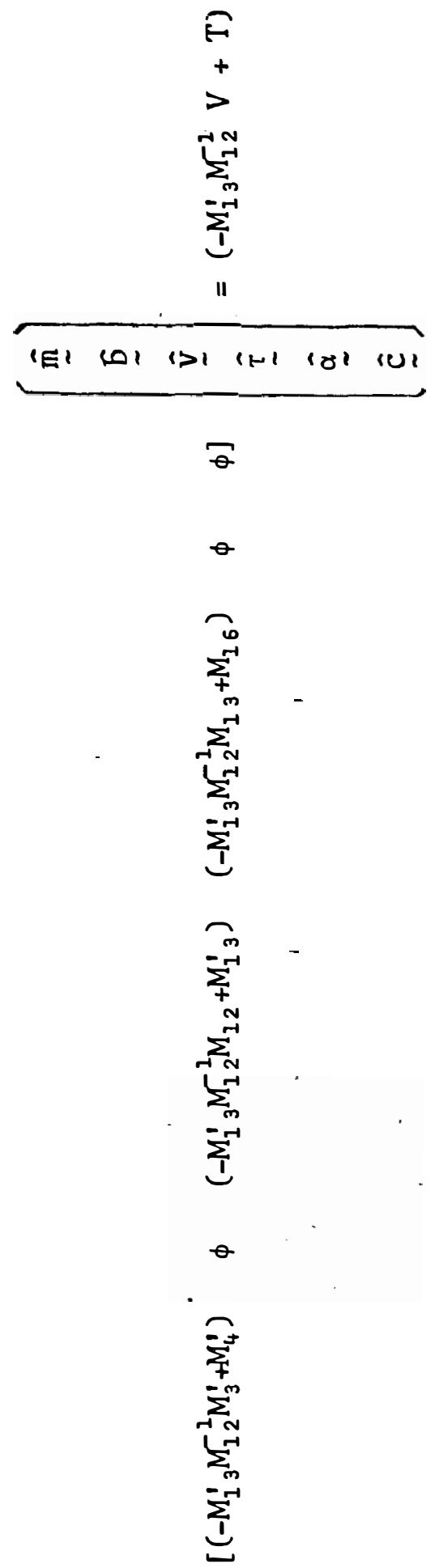




$$
\begin{gathered}
\text { Como }\left(-M_{13}^{\prime} M_{12}^{-1} M_{12}+M_{13}^{\prime}\right)=\phi \text {, resultou: } \\
\left(-M_{13}^{\prime} M_{12}^{-1} M_{3}^{\prime}+M_{4}^{\prime}\right) \hat{m}+\left(-M_{13}^{\prime} M_{12}^{-1} M_{13}+M_{16}\right) \hat{I}=\left(-M_{13}^{\prime} M_{12}^{-1} V+T\right), \\
\text { o tambêm: } \quad\left(-M_{13}^{\prime} M_{12}^{-1} M_{3}^{\prime}+M_{4}^{\prime}\right)=\phi \text {, então: } \\
\left(-M_{13}^{\prime} M_{12}^{-1} M_{13}+M_{26}\right) \hat{I}=\left(-M_{13} M_{12}^{-1} V+T\right) .
\end{gathered}
$$

Fazendo:

$$
\left(-M_{13} M_{12}^{-1} M_{13}+M_{16}\right)=C^{*}
$$

e

$$
\left(-M_{13}^{\prime} M_{12}^{-1} V-T\right)=Q^{*},
$$

obtivemos:

$$
C^{*} \hat{\underline{\tau}}=Q^{*} \quad \text {, }
$$

que é o sistema de equações normais para tratamentos eli minados os efeitos de vacas.

Como a matriz $C^{*}$ tem característica igual a (p-1), uma alternativa segundo CHAKRABARTI (1962), è introduzir uma restrição naão estimável nas soluçōes.

Sendo assim, obtemos um novo sistema de equa çōes :
$A \tilde{\tau}=\phi$ 
68.

Sominilo-se (V) em (IV), obtemos:

$$
\left(C^{*}+A\right) \cdot \tilde{\tau}=Q^{*}
$$

Senclo $C^{*}+A=N^{*}$, logo:

$$
N^{*} \underline{\underline{\tau}}=Q^{*}
$$

Como por hipótese, a matriz $N^{*} \vec{e}$ não singular, temos:

$$
\underline{\mathrm{T}}=\dot{N}^{*} 1 Q^{*}
$$

mando-se a matriz A como:

Admitindo-se a restrição $\underset{k=1}{\frac{p}{\Sigma}} \hat{t}_{k}=0$, e to-

$$
A=\frac{1}{J}\left[\begin{array}{lll}
\lambda_{2} & \lambda_{2} & \lambda_{2} \\
\lambda_{2} & \lambda_{2} & \lambda_{2} \\
\lambda_{2} & \lambda_{2} & \lambda_{2}
\end{array}\right]
$$

onde $\lambda_{2}=\sum_{i, k}\left(a_{i}^{\prime}, k^{\prime} \cdot a_{i, k^{\prime}}\right)$ para $i \neq i^{\prime}$ e $k \neq k^{\prime}$ e $a_{i k} \vec{e} o$ nümero de vezes que o animal i recebeu o tratamento $\underline{\mathrm{k}}$ on de $k=1,2, \ldots, p$ e $i=1,2, \ldots, v$, vem:

$$
N^{*}=\left(r_{k}-\frac{\lambda_{1}}{J}+\frac{\lambda_{2}}{J}\right) I_{(p)},
$$

e $\lambda_{1}=\sum_{i, k} a_{i, k}^{2}$ para $i=i^{\prime} e k=k^{\prime}$, então, no exemplo em questāo: 


$$
\begin{aligned}
& N^{*}=\left(6-\frac{10}{3}+\frac{4}{3}\right) I_{(3)}=4 \cdot I_{(3)} \\
& N^{*}=4 \cdot I_{(3)} \\
& N^{ \pm 1}=\frac{1}{4} I_{(3)}
\end{aligned}
$$

Sendo $Q^{*}=\left(-M_{13}^{1} M_{12}^{-1} V+T\right)$, resulta em:

$$
Q^{*}=\left[\begin{array}{l}
T_{1}-\frac{2}{3}\left(V_{1}+V_{6}\right)-\frac{1}{3}\left(V_{3}+V_{4}\right) \\
T_{2}-\frac{2}{3}\left(V_{2}+V_{4}\right)-\frac{1}{3}\left(V_{1}+V_{5}\right) \\
T_{3}-\frac{2}{3}\left(V_{3}+V_{5}\right)-\frac{1}{3}\left(V_{2}+V_{6}\right)
\end{array}\right]
$$

\section{Portanto:}

$$
\left(\begin{array}{l}
\hat{\mathrm{t}}_{1} \\
\hat{\mathrm{t}}_{2} \\
\hat{\mathrm{t}}_{3}
\end{array}\right)=\frac{1}{4}\left(\begin{array}{l}
\mathrm{T}_{1}-\frac{2}{3}\left(\mathrm{~V}_{1}+\mathrm{V}_{6}\right)-\frac{1}{3}\left(\mathrm{~V}_{3}+\mathrm{V}_{4}\right) \\
\mathrm{T}_{2}-\frac{2}{3}\left(\mathrm{~V}_{2}+\mathrm{V}_{4}\right)-\frac{1}{3}\left(\mathrm{~V}_{1}+\mathrm{V}_{5}\right) \\
\mathrm{T}_{3}-\frac{2}{3}\left(\mathrm{~V}_{3}+\mathrm{V}_{5}\right)-\frac{1}{3}\left(\mathrm{~V}_{2}+\mathrm{V}_{6}\right)
\end{array}\right)
$$

que è a solução do sistema para tratamentos, eliminandoise os efeitos de vacas. 


\subsection{3 - Solução do sistema para vacas}

Do sub-sistema 1, temos:

$$
M_{3}^{\prime} \hat{\mathrm{m}}+M_{12} \underline{\hat{\mathrm{v}}}+M_{13} \hat{\underline{\tau}}=V \text {, }
$$

portanto:

$$
M_{12} \hat{v}=V-M_{13} \hat{\tau}-M_{3}^{\prime} \hat{m} .
$$

Sendo $M_{12}$ não singular, obtivemos:

$$
\hat{v}=M_{12}^{-1} V-M_{12}^{-1} M_{13} \hat{\tau}-M_{12}^{-1} M_{3}^{\prime} \hat{m},
$$

que è a solução do sistema para vacas em função da média

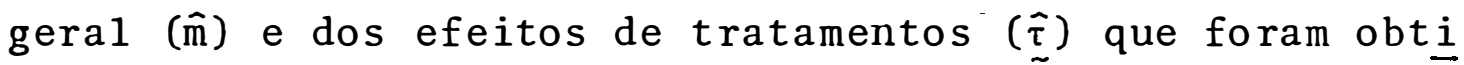
dos em 4.3.1 e 4.3.2, respectivamente.

$$
\left[\begin{array}{c}
\hat{v}_{1} \\
\hat{v}_{2} \\
\hat{v}_{3} \\
\hat{v}_{4} \\
\hat{v}_{5} \\
\hat{v}_{6}
\end{array}\right]=\frac{1}{3}\left[\begin{array}{l}
v_{1}-3 \hat{m}-2 \hat{t}_{1}-\hat{t}_{2} \\
v_{2}-3 \hat{m}-2 \hat{t}_{2}-\hat{t}_{3} \\
v_{3}-3 \hat{m}-\hat{t}_{1}-2 \hat{t}_{3} \\
v_{4}-3 \hat{m}-\hat{t}_{1}-2 \hat{t}_{2} \\
v_{5}-3 \hat{m}-\hat{t}_{2}-2 \hat{t}_{3} \\
v_{6}-3 \hat{m}-2 \hat{t}_{1}-\hat{t}_{3}
\end{array}\right]
$$

Esses resultados coincidem com aqueles obtidos por GODOI (1971), que estudou um modelo matemático se melhante sem considerar efeitos de blocos. 
71.

\subsection{4 - Solução do sistema para efeitos linear e quadrâtico de períodos}

Tomando-se a segunda equação do sub-sistema 2 ,

temos:

$$
M_{19} \underset{\sim}{\hat{\alpha}}=P \text {. }
$$

Como $M_{19}$ é não singular, existe $M_{19}^{-1}$ tal que $\mathrm{N}_{19}^{-1} M_{19}=\mathrm{I}$, logo:

$$
\hat{\alpha}=M_{19}^{-1} \cdot P \text {, }
$$

e entāo:

$$
\begin{aligned}
& \hat{\alpha}_{1}=\frac{1}{v \sum x_{j}^{2}}\left(P_{3}-P_{1}\right) \\
& \hat{\alpha}_{2}=\frac{1}{v \sum z_{j}^{2}}\left(P_{1}-2 P_{2}+P_{3}\right) .
\end{aligned}
$$

Assim, para o exemplo, vem

$$
\begin{aligned}
& \hat{\alpha}_{1}=\frac{1}{12}\left(P_{3}-P_{1}\right) \\
& \hat{\alpha}_{2}=\frac{1}{36}\left(P_{1}-2 P_{2}+P_{3}\right) .
\end{aligned}
$$

Esses resultados coincidem com as fórmulas ob tidas por GODOI (1971). 
4.3.5 - Solução do sistema para efeitos de blocos

Do sub-sistema 2 temos:

$$
\begin{aligned}
& M, \underline{\underline{b}}+M_{10} \underset{\sim}{\hat{\alpha}}=B \\
& M_{2} \underline{\underline{G}}=B-M_{10} \underset{\alpha}{\hat{\alpha}} .
\end{aligned}
$$

Sendo $M_{7}$ não singular, existe $M_{7}^{-1}$ tal que $M_{7}^{-1} M_{7} \approx I$, portanto, pré-multiplicando ambos os membros por $\mathrm{M}_{7}^{-3}$, obtém-se:

$$
\begin{aligned}
& M_{7}^{-1} M_{7} \underline{\underline{G}}=M_{7}^{-1} B-M_{7}^{-1} M_{10} \underset{\sim}{\alpha} \\
& \underline{\underline{b}}=\mathrm{M}_{7}^{-1} B-M_{7}^{-1} M_{10} \underset{\sim}{\hat{\alpha}} \text {, }
\end{aligned}
$$

que é a solução do sistema para blocos em função dos efe tos de períodos obtidos em 4.3.4.

$$
\begin{aligned}
& \sigma_{\ell}=\frac{1}{m_{\ell} \Sigma z_{j}^{2}} B_{\ell}-\hat{\alpha}_{2} \\
& \bar{b}_{\ell}=\frac{1}{18} \cdot B_{\ell}-\frac{1}{36}\left(P_{1}-2 P_{2}+P_{3}\right) .
\end{aligned}
$$


4.3.6 - Solução do sistema para efeitos da interașiño Período (1inear) $x$ Vacas

Ainda do segundo sub-sistema temos:

$$
\begin{aligned}
& M_{20}^{\prime} \hat{\alpha}+M_{21} \hat{\underline{c}}=C \\
& M_{21} \hat{\underline{c}}=C-M_{20}^{\prime} \underline{\alpha}
\end{aligned}
$$

Sendo $M_{21}$ não singular, vem:

$$
\begin{aligned}
\hat{c} & =M_{21}^{-1} C-M_{21}^{-1} M_{20}^{\prime} \hat{\alpha} \\
\hat{c}_{i} & =\frac{1}{\sum x_{j}^{2}}\left(y_{i 3 k \ell}-y_{i_{1} k l}\right)-\hat{\alpha}_{1} .
\end{aligned}
$$

Para o exemplo em questão, temos:

$$
\begin{aligned}
& \hat{c}_{1}=\frac{1}{2}\left(y_{1311}-y_{1111}\right)-\hat{\alpha}_{1} \\
& \hat{c}_{2}=\frac{1}{2}\left(y_{2321}-y_{2121}\right)-\hat{\alpha}_{1}
\end{aligned}
$$$$
\hat{c}_{3}=\frac{1}{2}\left(y_{3331}-y_{3131}\right)-\hat{\alpha}_{1}
$$$$
\hat{c}_{4}=\frac{1}{2}\left(\hat{y}_{4322}-y_{4122}\right)-\hat{\alpha}_{1}
$$$$
\hat{c}_{5}=\frac{1}{2}\left(y_{5332}-y_{5132}\right)-\hat{\alpha}_{1}
$$$$
\hat{c}_{6}=\frac{1}{2}\left(y_{6312}-y_{6112}\right)-\hat{\alpha}_{1}
$$ 
com $\hat{a}_{1}=\frac{1}{12}\left(P_{3}-P_{1}\right)$, conforme 4.3.4.

Esses resultados coincidem tambêm com os ob-

tidos por GODOI (1971).

4.4 - Somas de Quadrados

Com base no modelo adotado em (4.2), e toman

do-so:

$$
S Q R=y^{\prime} y-\hat{B}^{\prime} X^{\prime} y,
$$

onde $\hat{\beta}^{\prime} X^{\prime} y$ é a soma de quadrados dos parâmetros, definida Como:

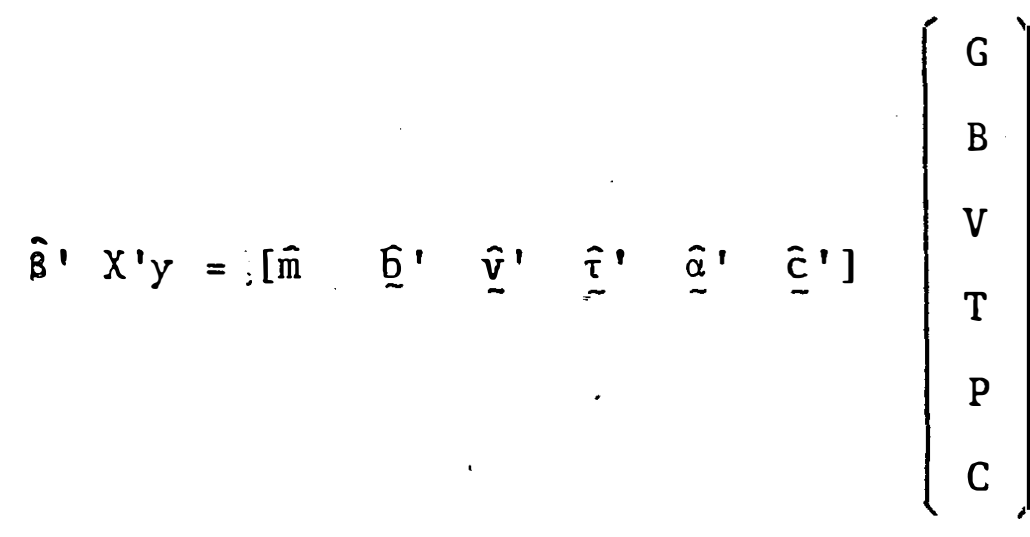

$$
\hat{\beta}^{\prime} X^{\prime} y=\left[\hat{m} G+\underline{b}^{\prime} \underline{\underline{v}}+\underline{\hat{v}}^{\prime} V+\hat{\tau}^{\prime} T+\hat{\alpha}^{\prime} P+\hat{c}^{\prime} C\right],
$$

que, através de (4.3), resultou: 


$$
\begin{aligned}
\hat{\beta}^{\prime} \cdot X^{\prime} y= & G^{\prime} M_{1}^{-1} G+\left[B^{\prime}\left(M_{7}^{-1}\right)^{\prime} B-\hat{\alpha}^{\prime} M_{10}^{\prime} M_{7}^{-1}, B\right]+ \\
& +\left[V^{\prime} M_{12}^{-1}, V-\hat{\tau}^{\prime} M_{13}^{\prime} M_{12}^{-1} V-\hat{m} M_{3}\left(M_{12}^{-1}\right)^{\prime} V\right]+(4.4 . a) \\
& +\hat{\tau}^{\prime} T+P^{\prime} M_{19}^{-1} P+\left[C^{\prime} M_{21}^{-1}, C-\hat{\alpha}_{\sim}^{\prime} M_{20}\left(M_{21}^{-1}\right)^{\prime} C\right] .
\end{aligned}
$$

Reunindo os termos onde aparece o vetor dos. efeitos de tratamentos $\hat{\tau}^{\prime}$, resultou:

$$
\begin{aligned}
S Q R= & y^{\prime} y-G^{\prime} M_{1}^{-1} G-\left[B^{\prime} M_{7}^{-1}, B-\hat{\alpha}^{\prime} M_{10}^{\prime}\left(M_{7}^{-1}\right)^{\prime} B\right]- \\
& -\left[V^{\prime} M_{12}^{-1}, V-\hat{m} M_{3}\left(M_{12}^{-1}\right)^{\prime} V\right]-\hat{\tau}\left[T-M_{13}^{\prime} M_{12}^{-1} V\right]-\quad(4.4 . b) \\
& -P^{\prime} M_{19}^{-1} P-\left[C^{\prime} M_{21}^{-1} C C-\hat{\alpha}^{\prime} M_{20} M_{21}^{-1} C\right],
\end{aligned}
$$

onde :

4.4.1 - Soma de quadrados devida à média:

$$
S Q(\hat{m})=G^{\prime} M_{1}^{-1} G=\frac{G^{2}}{N}
$$

4.4.2 - Soma de quadrados devida aos efeitos de blocos

SQ Blocos $=\mathrm{B}^{\prime}\left(\mathrm{M}_{7}^{-1}\right)^{\prime} \mathrm{B}-\underline{\alpha}^{\prime} \cdot \mathrm{M}_{I_{0}}^{\prime}\left(\mathrm{M}_{7}^{-1}\right)^{\prime} \mathrm{B}$ 
76.

$$
\begin{array}{r}
\text { onde, } B^{\prime}\left(M_{,}^{-2}\right)^{\prime} B=\frac{1}{\sum z_{j}^{2}} \sum_{\ell=1}^{L} \frac{1}{m_{\ell}} B_{\ell}^{2}, e \\
\hat{\alpha}^{\prime} M_{1}^{\prime}\left(M_{7}^{-3}\right)^{\prime} B=\hat{\alpha}_{2}\left(B_{1}+B_{2}\right)
\end{array}
$$

Por (4.3.4) temos que:

$$
\bar{\alpha}_{2}=\frac{\left(P_{1}-2 P_{2}+P_{3}\right)}{v \Sigma \Sigma_{j}^{2}}
$$

e, como:

$$
P=X_{(2)}^{\prime} y_{(2)}=\left(\begin{array}{rrrrrrrrrr}
-1 & 0 & 1 & -1 & 0 & 1 & \ldots & -1 & 0 & 1 \\
1 & -2 & 1 & 1 & -2 & 1 & \ldots & 1 & -2 & 1
\end{array}\right)_{(N)} \cdot(N) y_{(1)}=\left(\begin{array}{l}
-P_{1}+P_{2} \\
P_{1}-2 P_{2}+P_{3}
\end{array}\right)
$$

e

$$
B=X_{2}^{1} y=\underset{(2)}{\left(\begin{array}{rrrrrrrrrrrrrr}
1 & -2 & 1 & \ldots & 1 & -2 & 1 & 0 & 0 & 0 & \ldots & 0 & 0 & 0 \\
0 & 0 & 0 & \ldots & 0 & 0 & 0 & 1 & -2 & 1 & \ldots & 1 & -2 & 1
\end{array}\right)_{(N)} \cdot(N)^{y}(1)}=\left(\begin{array}{l}
B_{1} \\
B_{2}
\end{array}\right)
$$

temos que:

$$
\mathrm{B}_{1}+\mathrm{B}_{2}=\mathrm{P}_{1}-2 \mathrm{P}_{2}+\mathrm{P}_{3} \text {, }
$$

portanto, $\hat{\alpha}_{2}$ pode ser escrito na forma:

$$
\hat{\alpha}_{2}=\frac{\left(B_{1}+B_{2}\right)}{v \sum z_{j}^{2}},
$$


77.

logo,

$$
a^{\prime} M_{10}\left(M_{7}^{-1}\right)^{\prime} B=\frac{\left(B_{1}+B_{2}\right)^{2}}{v \sum z_{j}^{2}} \text {. }
$$

Então, a $S Q B l o c o s$ resultou:

$$
\text { SQ Blocos }=\frac{1}{\sum z_{j}^{2}} \sum_{\ell=1}^{L} \frac{1}{m_{\ell}} B_{\ell}^{2}-\frac{\left(\sum_{\ell=1}^{L} B_{\ell}\right)^{2}}{v \sum z_{j}^{2}}
$$

Podemos estabelecer analogia com a fórmula para SQ Blocos apresentada por LUCAS (1956), pois:

$$
B_{1}=D_{1}+D_{2}+D_{3}
$$

e

$$
\mathrm{B}_{2}=\mathrm{D}_{4}+\mathrm{D}_{5}+\mathrm{D}_{6},
$$

$\operatorname{logo}, B_{1}+B_{2}=\sum D_{i}$, portanto:

$$
\frac{\left(B_{1}+B_{2}\right)^{2}}{v \Sigma z_{j}^{2}}=\frac{\left(\Sigma D_{i}\right)^{2}}{3 n p(p-1)}=C_{B} \text {, }
$$

conforme LUCAS (1956), onde, segundo o autor, $n=2 R$ e $R$ è o número de repetiçōes de cada sequência.

A fórmula para a soma de quadrados de blocos apresentada por ele, sem demonstrações, é a seguinte :

$$
\text { SQ Blocos }=\frac{1}{6} \sum_{\ell=1}^{L} \frac{1}{m_{\ell}} B_{\ell}^{2}-\frac{\left(\Sigma D_{i}\right)^{2}}{3 n p(p-1)} \text {. }
$$


78.

4.4.3 - Soma de quadrados devida aos efeitos do vacas, ignorando efeitos de tratamentos

Através da soma de quadrados de resíduo $(4.4 . b)$, temos que:

$S Q$ Vacas (ign.trat.) $=\left[V^{\prime}\left(M_{12}^{-1}\right)^{\prime} V-\hat{m} M_{3}\left(M_{12}^{-1}\right) \cdot V\right]$.

Como:

$$
\text { mi } M_{3} M_{12}^{-1}, V=\frac{G^{2}}{N},
$$

obtivemos o seguinte resultado:

$$
\begin{aligned}
-\mathrm{SQ} \text { Vacas (ign.trat.) } & =V^{\prime} M_{12}^{-1} V-\hat{m} M_{3}\left(M_{12}^{-1}\right)^{\prime} V \\
& =V^{\prime} M_{12}^{-1} V-\frac{G^{2}}{N} \\
& =\frac{1}{J} \sum_{i=1}^{V} V_{i}^{2}-\frac{G^{2}}{N} .
\end{aligned}
$$

4.4.4 - Soma de quadrados devida a efeitos de tra tamentos ajustada para vacas

$$
\text { De (4.4.b) temos que: }
$$

SQ Trat. $(a j \cdot)=\hat{\tau}^{\prime}\left(-M_{13}^{\prime} M_{12}^{-1} V+T\right)$.

$$
\begin{gathered}
\text { Em }(4.3 .2) \text { vimos que: } \\
Q^{*}=-M_{13}^{1} M_{12}^{-1} V+T,
\end{gathered}
$$


que para o exemplo em estudo resultou em:

$$
\left.\begin{array}{l}
Q_{1}^{*}=T_{3}-\frac{2}{3}\left(V_{1}+V_{6}\right)-\frac{1}{j}\left(V_{3}+V_{4}\right) \\
Q_{2}^{*}=T_{2}-\frac{2}{3}\left(V_{2}+V_{4}\right)-\frac{1}{j}\left(V_{1}+V_{5}\right) \\
Q_{3}^{*}=T_{3}-\frac{2}{3}\left(V_{3}+V_{5}\right)-\frac{1}{j}\left(V_{2}+V_{6}\right)
\end{array}\right)
$$

logo,

$S Q \operatorname{Trat} \cdot(a j \cdot)=\hat{I}^{\prime} \cdot\left(-M_{13}^{\prime} M_{12}^{-1} V+T\right)=\tilde{\tau}^{\prime} Q$.

Podellos, também aqui, estabelecer una analogia coll a fórmula da SQ Tratamentos apresentada por LUCAS (1956).

o quadro a seguir serve para auxiliar a comparação desejada.

\begin{tabular}{ccccc}
\hline VACAS & \multicolumn{3}{c}{ PERIODOS } & $D_{i}$ \\
\cline { 2 - 4 } BIOCO 1 & I & II & III & \\
1 & & & & \\
2 & $y_{1111}$ & $y_{1222}$ & $y_{1311}$ & $y_{1111}-2 y_{1222}+y_{1311}$ \\
3 & $y_{2121}$ & $y_{2231}$ & $y_{2321}$ & $y_{2121}-2 y_{2231}+y_{2321}$ \\
BIOCO 2 & $y_{3131}$ & $y_{3211}$ & $y_{3331}$ & $y_{3131}-2 y_{3211}+y_{3331}$ \\
4 & & & & \\
5 & $y_{4122}$ & $y_{4212}$ & $y_{4322}$ & $y_{4122}-2 y_{4212}+y_{4322}$ \\
6 & $y_{5132}$ & $y_{5222}$ & $y_{5332}$ & $y_{5132}-2 y_{5222}+y_{5332}$ \\
5 & $y_{6112}$ & $y_{6232}$ & $y_{6312}$ & $y_{6112}-2 y_{6232}+y_{6312}$ \\
\hline
\end{tabular}


LUCAS (1956) apresenta a fórmula da soma de quadrados de tratamentos para $\mathrm{p}$ tratamentos:

$$
\text { SQ Trat. }\left(a j_{.}\right)=\frac{1}{6 n p} \sum_{k=1}^{p} Q_{k}^{2} \text {, }
$$

onde,

$$
\begin{aligned}
Q_{k}= & \text { soma dos } D^{\prime} \text { s em que o tratamento } \underline{k} \text { aparece nos } \\
& 19 \text { e } 39 \text { períodos, subtraída da soma dos } D^{\prime} s \text { em } \\
& \text { que o tratamento } \underline{\mathrm{k}} \text { aparece no } 29 \text { período; } \\
\mathrm{n}= & 2 \mathrm{R} ; \\
\mathrm{R}= & \mathrm{n} \stackrel{\text { de repetições de cada sequência. }}{ }
\end{aligned}
$$

Nas equações $\left(Q_{k}^{*}\right)$ de (VII), ao substituirmos os totais de vacas $\left(V_{i}\right)$ e os totais de tratamentos $\left(T_{k}\right)$ em função das observações $y_{i j k_{\ell}}$, de acordo com o quadro acima, resultou:

$$
\begin{aligned}
& Q_{1}^{*}=\frac{1}{3}\left[\left(D_{1}+D_{6}\right)-\left(D_{3}+D_{4}\right)\right]=\frac{1}{3} Q_{1} \\
& Q_{2}^{*}=\frac{1}{3}\left[\left(D_{2}+D_{4}\right)-\left(D_{1}+D_{5}\right)\right]=\frac{1}{3} Q_{2} \\
& Q_{3}^{*}=\frac{1}{3}\left[\left(D_{3}+D_{5}\right)-\left(D_{2}+D_{6}\right)\right]=\frac{1}{3} Q_{3} \\
& \quad \text { Como em }(\text { VIII }), \text { temos: } \\
& E_{1}=\frac{1}{4} Q_{1}^{*}=\frac{1}{4} \cdot \frac{1}{3} Q_{1}=\frac{1}{12} Q_{1} \\
& E_{2}=\frac{1}{4} Q_{2}^{*}=\frac{1}{4} \cdot \frac{1}{3} Q_{2}=\frac{1}{12} Q_{2} \\
& E_{3}=\frac{1}{4} Q_{3}^{*}=\frac{1}{4} \cdot \frac{1}{3} Q_{3}=\frac{1}{12} Q_{3}
\end{aligned}
$$


logo, a formula obtida neste trabalho para a soma de quadrados le tratamentos resultou em:

SQ Trat. (aj.) $=\underline{\tilde{I}}^{\prime} Q^{*}=\frac{1}{4} Q^{* \prime} Q^{*}=\frac{1}{4} \sum_{k=1}^{p} Q_{k}^{2}=\frac{1}{4} \sum_{k=1}^{p}\left(\frac{1}{j} Q_{k}\right)^{2}$

$$
\text { SQ Prat. (aj.) }=\frac{1}{4} \cdot \frac{1}{9} \sum_{k=1}^{p} Q_{k}^{2}=\frac{1}{36} \sum_{k=1}^{p} Q_{k}^{2} \text {, }
$$

que coincide com a proposta por LUCAS (1956). Para o exem plo em questão, onde $\mathrm{p}=3$ e $\mathrm{n}=2$, resultou:

SQ Trat. (aj.) $=\frac{1}{6.2 .3} \sum_{k \neq}^{\mathrm{p}} Q_{\mathrm{k}}^{2}=\frac{1}{36} \sum_{\mathrm{k} \neq}^{\mathrm{p}} \mathrm{Q}_{\mathrm{k}}^{2}$.

4.4.5 - Soma de quadrados devida a efeitos de pe. ríodos

De (4.4.b) obtemos:

SQ Períodos $=\mathrm{P}^{\prime} \mathrm{M}_{19}^{-1} \mathrm{P}={\underset{\sim}{\alpha}}^{\prime} \mathrm{P}$

SQ Periodos $=\hat{\alpha}_{1}\left(P_{3}-P_{1}\right)+\hat{\alpha}_{2}\left(P_{1}-2 P_{2}+P_{3}\right)$

que, por (4.3.4) ficou:

SQ Períodos $=\frac{\left(P_{3}-P_{1}\right)^{2}}{v \Sigma x_{j}^{2}}+\frac{\left(P_{1}-2 P_{2}+P_{3}\right)^{2}}{v \Sigma z_{j}^{2}}$

$$
=\frac{1}{v} \sum_{j=1}^{3} P_{j}^{2}-\frac{G^{2}}{N}
$$




$$
\text { SQ Períodos }=\frac{1}{6} \sum_{j=1}^{3} P_{j}^{2}-\frac{G^{2}}{18}
$$

onde:

$$
\begin{aligned}
\text { SQ Periodo (1inear) } & =S Q\left(\hat{\alpha}_{1}\right)=\frac{\left(P_{3}-P_{1}\right)^{2}}{v \Sigma x_{j}^{2}}=\frac{\left(P_{3}-P_{1}\right)^{2}}{12} \\
S Q \text { Periodos (quadrático) } & =S Q\left(\hat{\alpha}_{2}\right)=\frac{\left(P_{1}-2 P_{2}+P_{3}\right)^{2}}{v \Sigma z_{j}^{2}}=\frac{\left(P_{1}-2 P_{2}+P_{3}\right)^{2}}{36}
\end{aligned}
$$

Esses resultados coincidem com as fôrmulass apresentadas por BRANDT (1938) e GODOI (1971).

$$
\begin{aligned}
& \text { 4.4.6 - Soma de quadrados devida à interação Pe- } \\
& \text { ríodo (linear) } x \text { Vacas }
\end{aligned}
$$

SQ Int. Período (1inear) $x$ Vacas, $=\mathrm{C}^{\prime} \mathrm{M}_{21}^{-1}, \mathrm{C}-\underset{\sim}{\hat{\alpha}^{\prime}} \mathrm{M}_{20} \mathrm{M}_{21}^{-1} \mathrm{C}_{-}$

$$
\begin{aligned}
& \text { SQ Int. Período (1inear) } x \text { Vacas, }=\frac{1}{\sum x_{j}^{2}} \bar{i}\left(y_{i 3 k \ell}-y_{i_{-} k_{l}}\right)^{2}- \\
& -\frac{1}{v \Sigma x_{j}^{2}}\left(P_{3}-P_{1}\right)^{2} \text {, }
\end{aligned}
$$

que, para o exemplo em estudo, resultou:

SQ Int. Período (linear) $x$ Vacas $=\frac{1}{2} \sum_{i}\left(y_{i 3 k \ell}-y_{i 1 k \ell}\right)^{2}-\frac{1}{12}\left(P_{3}-P_{1}\right)^{2}$ 
Os resultados dos itens $4.4 .3,4.4 .4$ e 4.4 .6 coincidem com os obtidos por GODOI (1971).

\section{5 - Esperanças Matemáticas}

Para a obtenção das esperanças matemáticas, utilizamos o modelo tipo I ou modelo de efeitos fixos:

4.5.1 - Esperança matemática de $\mathrm{B}^{\prime} \mathrm{M}_{7}^{-1} \mathrm{~B}$.

Como B está representando $X_{2}^{\prime} y$, então:

$$
\begin{aligned}
& B^{\prime} M_{7}^{-1} B=y^{\prime} X_{2} M_{7}^{-1} X_{2}^{\prime} y \\
& E B^{\prime}\left(M_{7}^{-1}\right)^{\prime} B=E\left(y^{\prime} X_{2} M_{7}^{-1} X_{2}^{\prime} y\right)
\end{aligned}
$$

e, no modelo temos:

$$
\left\{\begin{array}{l}
Y=X \beta+\varepsilon \\
\varepsilon \cap N\left(\phi, \sigma^{2} I\right) \\
e \quad \text { independentes }
\end{array}\right.
$$

$$
\begin{aligned}
& E\left(\beta^{\prime} M_{7}^{-1} B\right)=E\left[\left(\beta^{\prime} X^{\prime}+\varepsilon^{\prime}\right) X_{2} M_{7}^{-1} X_{2}^{\prime}\left(X_{\beta}+\varepsilon\right)\right] \\
& E\left(\beta^{\prime} M_{7}^{-1} B\right)=\beta^{\prime} X^{\prime} X_{2} M_{7}^{-1} X_{2}^{\prime} X_{\beta}+\sigma^{2} \operatorname{tr}\left(X_{2} M_{7}^{-1} X_{2}^{\prime}\right)
\end{aligned}
$$


sendo:

$$
X_{2}^{\prime} X=\left[\begin{array}{llllll}
\phi & M_{7} & \phi & \phi & M_{10} & \phi
\end{array}\right] .
$$

e

$$
\begin{aligned}
& B^{\prime}=\left[\begin{array}{llllll}
m & \underline{b}^{\prime} & \underline{v}^{\prime} & \underline{\tau}^{\prime} & \underline{\alpha}^{\prime} & \underline{c}^{\prime}
\end{array}\right] \\
& \beta^{\prime} X^{\prime} X_{2} M_{7}^{1} X_{2}^{\prime} X \beta=\left(\underline{b}^{\prime} M_{7}^{\prime}+\underline{\alpha}^{\prime} M_{10}^{\prime}\right) M_{7}^{-1}\left(M_{7} \underline{b}+M_{10} \underset{\sim}{\alpha}\right) \\
& \text { Sendo } M_{7} \text { simétrica, } M_{7}^{\prime}=M_{7} \text {, } \\
& =\underline{\sim}^{\prime} \mathrm{M}_{7} \underline{\mathrm{b}}+\underline{\sim}^{\prime} \mathrm{M}_{10} \stackrel{\alpha}{\alpha}+\underline{\alpha}^{\prime} \mathrm{M}_{10}^{\prime} \underset{\sim}{\mathrm{b}}+\underline{\alpha}^{\prime} \mathrm{M}_{10}^{\prime} \mathrm{M}_{7}^{-1} \mathrm{M}_{10} \stackrel{\alpha}{\sim}
\end{aligned}
$$

Como:

$$
\underline{\sim}^{\prime} \mathrm{M}_{10} \stackrel{\alpha}{\alpha}=\underline{\alpha}^{\prime} \mathrm{M}_{10}^{\prime} \underset{\sim}{\mathrm{b}}=\phi
$$$$
\beta^{\prime} X^{\prime} X_{2} M_{7}^{-1} \quad X_{2}^{\prime} \quad X \quad \beta=\underline{\sim}^{\prime} M_{7} \underset{b}{b}+\stackrel{\alpha}{\prime}^{\prime} M_{10}^{\prime} M_{7}^{-1} M_{10} \stackrel{\alpha}{\sim}
$$

Portanto:

$$
E\left[B^{\prime} M_{7}^{-1} B\right]=f_{1}(\beta)+L \sigma^{2}
$$

onde $f_{1}(\beta)=\underline{b}^{\prime} M_{7} \underset{\sim}{b}+\underline{\alpha}^{\prime} M_{10}^{\prime} M_{7}^{-1} M_{10} \stackrel{\alpha}{\sim}$. 


\section{5 .2 - Esperança matemätica de $V \cdot M_{12}^{-1} V$}

Como V estā representando $X_{3}^{\prime} y$, então:

$$
\begin{aligned}
& V^{\prime} M_{12}^{-1} V=y^{\prime} X_{3} M_{12}^{-1} X_{3}^{\prime} V^{\prime} \\
& E_{1}\left[V^{\prime} M_{12}^{-1} V\right]=E\left[y^{\prime} X_{3} M_{12}^{-1} X_{3}^{\prime} y\right]
\end{aligned}
$$

que, por (4.5.1.b), fica:

$$
E\left[V^{\prime} M_{12}^{-1} V\right]=\beta^{\prime} X^{\prime} X_{3} M_{12}^{-1} X_{3}^{\prime} X_{\beta}+\sigma^{2} \operatorname{tr}\left(X_{3} M_{12}^{-1} X_{3}^{\prime}\right) .
$$

Sendo:

$$
\begin{aligned}
& X_{3}^{\prime} X=\left[\begin{array}{llllll}
M_{3}^{\prime} & \phi & M_{12} & M_{13} & \phi & \phi
\end{array}\right]
\end{aligned}
$$

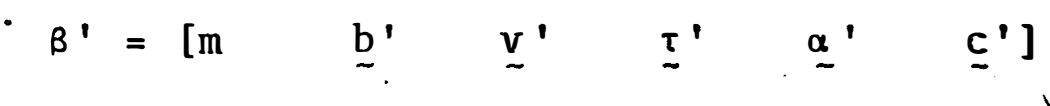

logo :

$$
\begin{aligned}
\beta^{\prime} X^{\prime} X_{3} M_{12}^{-1} X_{3}^{\prime} X_{\beta}= & \left(m M_{3}+\underline{v}^{\prime} M_{12}+\tau^{\prime} M_{13}^{\prime}\right) M_{12}^{-1}\left(M_{3}^{\prime} m+M_{12} \underline{v}+M_{13} \tau\right) \\
= & m M_{3} M_{12}^{-1} M_{3}^{\prime} m+m M_{3} M_{12}^{-1} M_{12} \underline{v}+ \\
& +m M_{3} M_{12}^{-1} M_{13} \underset{\sim}{\tau}+\underline{v}^{\prime} M_{12} M_{12}^{-1} M_{3}^{\prime} m+ \\
& +\underline{\sim}^{\prime} M_{12} M_{12}^{-1} M_{12} \underline{v}+\underline{v}^{\prime} M_{12} M_{12}^{-1} M_{13} \tau+ \\
& +\tau^{\prime} M_{13}^{\prime} M_{12}^{-1} M_{3}^{\prime} m+\tau^{\prime} M_{13}^{\prime} M_{12}^{-1} M_{12} \underline{v}+ \\
& +\sim^{\prime} M_{13}^{\prime} M_{12}^{-1} M_{13} \underline{\tau}
\end{aligned}
$$


86.

$$
\beta^{\prime} X ! X_{3} M_{12}^{-1} X_{3}^{\prime} X_{\beta}=N m^{2}+\underline{v}^{\prime} M_{12} \underline{v}+2 \underline{v}^{\prime} M_{13} \underline{\tau}+\tau_{\sim}^{\prime} M_{13}^{\prime} M_{12}^{-1} M_{13} \underline{\tau}
$$

\section{Portanto,}

$$
E\left(V^{\prime} M_{12}^{-1} V\right)=f_{2}(\beta)+V \sigma^{2}
$$

onde: $f_{2}(\beta)=N m^{2}+\underline{v}^{\prime} M_{12} \underset{\sim}{v}+2 \underline{v}^{\prime} M_{13} \underline{\tau}+\underline{\tau}^{\prime} M_{13}^{\prime} M_{12}^{-1} M_{13} \tau$.

$$
4.5 .3 \text { - Esperança matemática de } \hat{\tau}^{\prime} Q \text { * }
$$

$$
\begin{aligned}
& \text { Sendo } \hat{\tau}=N^{*} 1 Q^{*} \text { e } Q^{*}=W^{\prime} X^{\prime} y \text {, então: } \\
& \hat{\tau}^{\prime} Q^{*}=Q^{* \prime} N^{*_{1}} Q^{*}=y^{\prime} X W^{\prime} N^{* 1} W X \cap y \quad(4 \cdot 5.3 . a) \\
& E\left(\hat{\tau}^{\prime} Q^{*}\right)=E\left[y^{\prime} X W^{\prime} N^{\star} 1-W X^{\prime} y\right),
\end{aligned}
$$

que, por (4.5.1.b), fica:

$$
E\left(\hat{\tau}^{\prime} Q^{*}\right)=\beta^{\prime} X^{\prime} X W^{\prime} N^{ \pm_{1}} W X^{\prime} X \beta+E\left(\varepsilon^{\prime} X W^{\prime} N^{*_{1}} W X^{\prime} \varepsilon\right)
$$

Sendo vâlidas as propriedades abaixo, segundo PIMENTEL GOMES (1967) e IEMMA (1981),

$$
\begin{gathered}
W X^{\prime} X \beta=C^{*} \tau=Q^{*} \\
W X^{\prime} X W^{\prime}=C^{*}
\end{gathered}
$$

onde $W$ é a matriz definida em 4.3.2.

$$
\beta^{\prime} X^{\prime} X W^{\prime} N^{ \pm 1} W X^{\prime} X \beta=\tau^{\prime} C^{*} N^{ \pm}{ }^{1} C^{*} \tau
$$


e, desde que $C^{*} N^{{ }^{*} 1} C^{*}=C^{*} N^{\star}{ }^{1} C^{*}=C^{*}, e$, ainda, $C^{*} \tau=$ $=Q^{*}$, temos:

$$
B^{\prime} X^{\prime} X W^{\prime} N^{\star 1} W X^{\prime} X \beta=\tau^{\prime} C^{*} \underline{\tau}=\underline{\tau}^{\prime} Q^{*}
$$

e

$E\left[\varepsilon^{\prime} X W^{\prime} N^{\star 1} W X^{\prime} \varepsilon\right]=\sigma^{2} \operatorname{tr}\left(X W^{\prime} N^{\star 1} W^{\prime} X^{\prime}\right)$

Fazendo $X W^{\prime} \cdot N^{\star 1}=A_{1}$ e $W^{\prime}=B_{1}$, temos:

$\operatorname{tr}\left(A_{1} B_{1}\right)=\operatorname{tr}\left(B_{1} A_{1}\right)=\operatorname{tr}\left(W X^{\prime} X W^{\prime} N^{* 1}\right)$

que, por $(4.6 .3 . c)$, fica:

$$
\operatorname{tr}\left(W X^{\prime} X W^{\prime} N^{* 1}\right)=\operatorname{tr}\left(C^{*} N^{\star 1}\right)
$$

Então:

$$
E\left[\begin{array}{lllllll}
\varepsilon^{\prime} & X & W^{\prime} & N^{*} 1 & W & X^{\prime} & \varepsilon
\end{array}\right]=\sigma^{8} \operatorname{tr}\left(C^{*} N^{* 1}\right),
$$

como $C^{*} N^{\star} 1$ é uma matriz idempotente, pois

$$
C^{*} N^{*_{1}} C^{*} N^{* 1}=C^{*} N^{{ }_{1}} 1
$$

então,

$$
\operatorname{tr}\left(C^{*} N^{\star 1}\right)=r\left(C^{\star} N^{\star} 1\right)
$$

0 produto de uma matriz singular por uma matriz nīo singular preserva a característica da matriz sin gular: 


$$
\operatorname{tr}\left(C^{*} N^{*} 1\right)=r\left(C^{*} N^{\star} 1\right)=r\left(C^{*}\right)=p-1
$$

\section{Portanto,}

$$
E\left(\tilde{\tau}^{\prime} Q^{*}\right)=f_{3}(\beta)+(p-1) \sigma^{2}
$$

onde $f_{g}(\beta)=\underline{\tau}^{\prime} Q^{*}$.

4.5 .4 - Esperança matemática de $P^{\prime} M_{19}^{-1} P$

Como P está representando $X_{5}^{\prime} y$, então:

$$
\begin{gathered}
P^{\prime} M_{19}^{-1} P=y^{\prime} X_{5} M_{19}^{-1} X_{5}^{\prime} y \\
E\left(P^{\prime} M_{19}^{-1} P\right)=E\left[y^{\prime} X_{5} M_{19}^{-1} X_{5}^{\prime} y\right],
\end{gathered}
$$

que, por (4.5.1.b), fica:

$$
E\left[P^{\prime} M_{19}^{-1} P\right]=\beta^{\prime} X^{\prime} X_{5} M_{19}^{-1} X_{5}^{\prime} X \beta+\sigma^{2} \operatorname{tr}\left(X_{5} M_{19}^{-1} X_{5}^{\prime}\right) .
$$

Sendo:

$$
\begin{aligned}
& X_{5}^{\prime} X=\left[\begin{array}{llllll}
\phi & M_{10}^{\prime} & \phi & \phi & M_{19} & M_{20}
\end{array}\right] \\
& B^{\prime}=\left[\begin{array}{llllll}
m & \underline{b}^{\prime} & \underline{v}^{\prime} & \underline{\tau}^{\prime} & \underline{\alpha}^{\prime} & \underline{c}^{\prime}
\end{array}\right]
\end{aligned}
$$

logo: 


$$
\begin{aligned}
& B^{\prime} X^{\prime} X_{5} M_{19}^{-1} X_{5}^{\prime} X_{\beta}=\left(\underline{b}^{\prime} M_{10}^{\prime}+\underline{\alpha}^{\prime} M_{19}+c^{\prime} M_{20}^{\prime}\right) M_{19}^{-1}\left(M_{10}^{\prime} \underline{b}+M_{19} \underline{\alpha}+M_{20} \underline{c}\right) \\
& =\underline{b}^{\prime} M_{10} M_{19}^{-1} M_{10}^{\prime} \underline{b}+\underline{b}^{\prime} M_{10} \underline{a}+\underline{b}^{\prime} M_{20} M_{19}^{-1} M_{20} \underline{c}+ \\
& +\underline{\alpha}^{\prime} \mathrm{M}_{10}^{\prime} \underset{\sim}{b}+\underline{\alpha}^{\prime} \mathrm{M}_{19} \underline{\alpha}+\underline{\alpha}^{\prime} \mathrm{M}_{20} \underline{\mathrm{c}}+ \\
& +\underline{c}^{\prime} M_{20}^{\prime} \quad M_{19}^{-1} M_{10}^{\prime} \underline{b}+\underline{c}^{\prime} M_{20}^{\prime} \underline{\alpha+} \\
& +\underset{\sim}{C^{\prime}} M_{20}^{\prime} M_{19}^{1} M_{20} \stackrel{c}{\sim} \\
& =\underline{b}^{\prime} M_{10} M_{19}^{-1} M_{10}^{\prime} \underline{b}+\underline{\alpha}^{\prime} M_{19} \underline{\alpha}
\end{aligned}
$$

Portanto:

$$
E\left[P^{\prime} M_{19}^{-i} P\right]=f_{4}(\beta)+(J-1) \sigma^{2}
$$

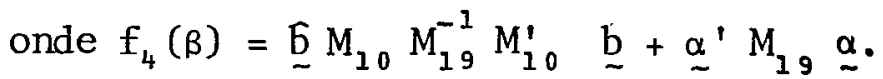

4.5.5- Esperança matemātica de $\mathrm{C}^{\prime} \mathrm{M}_{21}^{-1} \mathrm{C}$

Como C estā representando $X_{6}^{\prime} y$

$$
\begin{gathered}
C^{\prime} M_{21}^{-1} C=y^{\prime} X_{6} M_{21}^{1} X_{6}^{\prime} y^{\prime} \\
E\left[C^{\prime} M_{21}^{-1} C\right]=E\left[y^{\prime} X_{6} M_{2 I}^{1} X_{6}^{\prime} Y\right]
\end{gathered}
$$

que, por (4.5.1.b), fica:

$$
E\left[C^{\prime} M_{21}^{-1} C\right]=B^{\prime} X^{\prime} X_{6} M_{21}^{-1} X_{6}^{\prime} X \beta+\sigma^{2} \operatorname{tr}\left(X_{6} M_{21}^{-1} \lambda_{6}^{\prime \prime}\right)
$$


9).

Sendo:

$$
\begin{aligned}
& \mathrm{X}_{6}^{\prime} \mathrm{X}=\left[\begin{array}{lllllll}
\phi & \phi & \phi & \phi & \mathrm{M}_{20}^{\prime} & \mathrm{M}_{23}
\end{array}\right] \\
& B^{\prime}=\left[\begin{array}{lllll}
m & \underline{b}^{\prime} & \underline{v}^{\prime} & \underline{\tau}^{\prime} & \underline{\alpha}^{\prime}
\end{array}\right.
\end{aligned}
$$

então:

$$
\begin{aligned}
& \beta^{\prime} X^{\prime} X_{6} M_{21}^{-1} X_{6}^{\prime} X \beta=\left(\underline{\alpha}^{\prime} M_{20}+\underline{c}^{\prime} M_{21}^{\prime}\right) M_{21}^{-1}\left(M_{20}^{\prime} \underline{\alpha}+M_{21} \stackrel{c}{\sim}\right) \\
& =\underline{\alpha}^{\prime} \mathrm{M}_{20} \mathrm{M}_{21}^{-1} \mathrm{M}_{20}^{\prime} \stackrel{\alpha}{\alpha}+\underline{\alpha}^{\prime} \mathrm{M}_{20} \mathrm{M}_{21}^{-1} \mathrm{M}_{21} \underline{\mathrm{c}}+ \\
& +\stackrel{c}{\prime}^{\prime} M_{21}^{\prime} M_{21}^{-1} M_{20}^{\prime} \underset{\sim}{\alpha}+\stackrel{c}{\prime}^{\prime} M_{21}^{\prime} M_{21}^{-1} M_{21} \stackrel{c}{\sim}
\end{aligned}
$$

Sendo $M_{21}$ simëtrica, $M_{21}=M_{21}^{\prime}$

$$
\beta^{\prime} X^{\prime} X_{6} M_{21}^{-1} X_{6}^{\prime} X \beta=\hat{\alpha}_{\sim}^{\prime} M_{20} M_{21}^{-1} M_{20}^{\prime} \underline{\alpha}+\underline{c}^{\prime} M_{21} \stackrel{c}{c}
$$

Portanto:

$$
E\left[C^{\prime} M_{21}^{-1} C\right]=f_{5}(\beta)+v \sigma^{2}
$$

onde $f_{5}(\beta)=\stackrel{\alpha}{\prime}^{\prime} M_{20} M_{21}^{-1} M_{20}^{\prime} \stackrel{\alpha}{\sim}+\underset{c^{\prime}}{\prime} M_{21} \stackrel{c}{c}$.

\subsection{6 - Esperança matemätica de $y^{\prime} y$.}

De (4.5.1.b), temos:

$$
\begin{gathered}
y^{\prime} y=\left(\beta^{\prime} X^{\prime}+\varepsilon^{\prime}\right)(X \beta+\varepsilon) \\
E\left(y^{\prime} y\right)=E\left[\beta^{\prime} X^{\prime} X \beta+\beta^{\prime} X^{\prime} \varepsilon+\varepsilon^{\prime} X \beta+\varepsilon^{\prime} \varepsilon\right]
\end{gathered}
$$


91.

$$
\begin{aligned}
& E\left(y^{\prime} y\right)=\beta^{\prime} X^{\prime} X \beta+E\left(\varepsilon^{\prime} \varepsilon\right) \\
& E\left(y^{\prime} y\right)=\beta^{\prime} X^{\prime} X \beta+N \sigma^{2} .
\end{aligned}
$$

Sendo:

$$
\begin{gathered}
B^{\prime}=\left[\begin{array}{llllll}
\mathrm{m} & \underline{b}^{\prime} & \underline{v^{\prime}} & \underline{\tau}^{\prime} & \underline{\alpha}^{\prime} & \underline{c}^{\prime}
\end{array}\right] \\
\mathrm{X}^{\prime} \mathrm{X}=\left[\begin{array}{cccccc}
\mathrm{M}_{1} & \phi & \mathrm{M}_{3} & \mathrm{M}_{4} & \phi & \phi \\
\phi & \mathrm{M}_{7} & \phi & \phi & \mathrm{M}_{10} & \phi \\
\mathrm{M}_{3}^{\prime} & \phi & \mathrm{M}_{12} & \mathrm{M}_{13} & \phi & \phi \\
\mathrm{M}_{4}^{\prime} & \phi & \mathrm{M}_{13}^{\prime} & \mathrm{M}_{16} & \phi & \phi j \\
\phi & \mathrm{M}_{10}^{\prime} & \phi & \phi & \mathrm{M}_{19} & \mathrm{M}_{20} \\
\phi & \phi & \phi & \phi ! & \mathrm{M}_{20}^{\prime} & \mathrm{M}_{21}
\end{array}\right]
\end{gathered}
$$

Obtemos :

$$
\begin{aligned}
E\left(y^{\prime} y\right)=f_{6}(\beta)+N \sigma^{2}, \\
f_{6}(\beta)=N m^{2}+\underset{b^{\prime}}{M_{7}} \underline{b}+\underline{v}^{\prime} M_{12} \underline{v}+\underline{\tau}^{\prime} M_{16} \underline{\tau}+\underline{\alpha}^{\prime} M_{19} \underset{\sim}{\alpha}+ \\
+\underline{c}^{\prime} M_{21} \underline{c}+2 \underline{v}^{\prime} M_{13} \underline{\tau}+2 \underline{b}^{\prime} M_{10} \underline{\alpha}+ \\
+2 \underline{c}^{\prime} M_{20}^{\prime} \underline{\alpha}
\end{aligned}
$$


4.5.7 - Esperança matenātica de $\mathrm{M}_{1}^{-1}\left[y^{\prime} \mathrm{X}_{1} \mathrm{X}_{1}^{\prime} \mathrm{y}\right]$

$$
\begin{aligned}
M_{1}^{-1}\left[y^{\prime} X_{1} X_{1}^{\prime} y\right] & =\frac{1}{N}\left[y^{\prime} X_{1} X_{1}^{\prime} y\right]=\frac{1}{N} G^{2} \\
\frac{1}{N} E\left[G^{2}\right] & =\frac{1}{N} E\left[y^{\prime} X_{1} X_{1}^{\prime} y\right]
\end{aligned}
$$

que, por (4.5.1.b), fica:

$$
\begin{aligned}
\frac{1}{N} E\left(G^{2}\right) & =\frac{1}{N} E\left[\left(B^{\prime} X^{\prime}+\varepsilon^{\prime}\right) X_{1} X_{1}^{\prime}\left(X \beta+\varepsilon^{\prime}\right)\right. \\
& =\frac{1}{N}\left(\beta^{\prime} X^{\prime} X_{1} X_{1}^{\prime} X \beta\right)+\frac{1}{N} E\left[\varepsilon^{\prime} X_{1} X_{1}^{\prime} \varepsilon\right]
\end{aligned}
$$

$$
\begin{aligned}
& \text { Como } \\
& \beta^{\prime}=\left[\begin{array}{llllll}
\mathrm{m} & \underline{b}^{\prime} & \underline{v}^{\prime} & \underline{\tau}^{\prime} & \underline{\alpha}^{\prime} & \underline{c}^{\prime}
\end{array}\right] \\
& \mathrm{X}_{1}^{\prime} \mathrm{X}=\left[\begin{array}{llllll}
\mathrm{N} & \phi & \mathrm{M}_{3} & \mathrm{M}_{4} & \phi & \phi
\end{array}\right]
\end{aligned}
$$

temos:

$$
\begin{aligned}
\frac{1}{N}\left(\beta^{\prime} X^{\prime} X_{1} X_{1}^{\prime} X \beta\right)= & m^{2} N+2 m M_{3} \underset{\sim}{v}+2 m M_{4} \underset{\tau}{\tau}+ \\
& +\frac{1}{N}{\underset{\sim}{\tau}}^{\prime} M_{4}^{\prime} M_{4} \underset{\sim}{\tau}+\frac{1}{N} \underline{v}^{\prime} M_{3}^{\prime} M_{3} \underline{v}+ \\
& +\frac{2}{N} \underline{\sim}^{\prime} M_{3}^{\prime} M_{4} \underline{\tau}
\end{aligned}
$$


que, ao efetuarmos os produtos e aplicamos as restrições impostas ni solução do sistema de equações nomais, resulta:

$$
\begin{aligned}
& \frac{1}{N}\left(\beta^{\prime} X^{\prime} X_{1} X_{1}^{\prime} X \beta\right)=m^{2} \cdot N \\
& \text { Portanto, } \\
& E\left[M_{1}^{-1}\left(y^{\prime} X_{1} X_{1}^{\prime} y\right)\right]=E\left[G^{2} / N\right]=f_{7}(\beta)+\sigma^{2}
\end{aligned}
$$

onde $f_{7}(\beta)=m^{2} N$.

4.5.8 - Esperança matemática da correção para blocos

Segundo LUCAS (1956):

$$
C_{B}=\frac{\left(\Sigma D_{i}\right)^{2}}{3 n p(p-1)}=\frac{\left(B_{1}+B_{2}\right)^{2}}{v \sum z_{j}^{2}}=\frac{\left(P_{1}-2 P_{2}+P_{3}\right)^{2}}{v \sum z_{j}^{2}}
$$

Sendo:

$$
\begin{gathered}
\frac{\left(P_{1}-2 P_{2}+P_{3}\right)^{2}}{v \sum z_{j}^{2}}=\frac{1}{v \sum z_{j}^{2}}\left[y^{\prime} X_{2} E X_{2}^{\prime} y\right] \\
\frac{1}{v \sum z_{j}^{2}} E\left[P_{1}-2 P_{2}+P_{3}\right]^{2}=\frac{1}{v \sum z_{j}^{2}} E\left[y^{\prime} X_{2} E X_{2}^{\prime} y\right]
\end{gathered}
$$

que, por (4.5.1.b), fica:

$$
\frac{1}{v \Sigma z_{j}^{2}} E\left[y^{\prime} X_{2} E X_{2}^{\prime} y\right]=\beta^{\prime} X^{\prime} X_{2} E X_{2}^{\prime} X \beta+E\left[\varepsilon^{\prime} X_{2} E X_{2}^{\prime} \varepsilon\right] \text {. }
$$


Sendo:

$$
\begin{aligned}
& \mathrm{X}_{2}^{\prime} \mathrm{X}=\left[\begin{array}{llllll}
\phi & \mathrm{M}_{7} & \phi & \phi & \mathrm{M}_{10} & \phi
\end{array}\right] \\
& \beta^{\prime}=\left[\begin{array}{lllll}
m & \underline{b}^{\prime} & \underline{v}^{\prime} & \underline{\tau}^{\prime} & \underline{\alpha}^{\prime}
\end{array}\right.
\end{aligned}
$$

então:

$$
\begin{aligned}
& \beta^{\prime} X^{\prime} X_{2} E X_{2}^{\prime} X \beta=\left(\underline{b}^{\prime} M_{7}^{\prime}+\underline{\alpha}^{\prime} M_{10}^{\prime}\right) E\left(M_{7} \underline{b}+M_{10} \underline{\alpha}\right) \\
& =\underline{b}^{\prime} M_{7}^{\prime} E M_{7} \underline{b}+\underline{b}^{\prime} M_{7}^{\prime} E M_{10} \underline{\alpha}+\underline{\alpha}^{\prime} M_{10}^{\prime} E M_{7} \underset{b}{b}+ \\
& +\underset{\sim}{\alpha} \mathrm{M}_{10}^{1} \mathrm{E} \mathrm{M}_{10} \stackrel{\alpha}{\sim}
\end{aligned}
$$

Sendo $\sum_{\ell} \mathrm{b}_{\ell}=0$, uma das restrições impostas, resulta:

$$
\beta^{\prime} X^{\prime} X_{2} E X_{2}^{\prime} X \beta=\alpha^{\prime} M_{10}^{\prime} E M_{10} \stackrel{\alpha}{\sim}
$$

e

$$
\begin{aligned}
E\left[\varepsilon^{\prime} X_{2} E_{L} X_{2}^{\prime} \dot{\theta}\right] & =\sigma^{2} \operatorname{tr}\left(X_{2} E X_{2}^{\prime}\right)=\sigma^{2} \operatorname{tr}\left(X_{2}^{\prime} X_{2} E_{L}\right) \\
& =\sigma^{2} \cdot \operatorname{tr}\left(M_{7} E_{L}\right) \\
\operatorname{tr}\left(M_{7} E_{L}\right) & =\sigma^{2} \cdot m_{\ell} \cdot \sum z_{j}^{2} \cdot L=\sigma^{2} \cdot m_{\ell} \cdot \sum z_{j}^{2} \cdot(J-1)
\end{aligned}
$$

Portanto:

$$
\begin{aligned}
\frac{1}{v \sum z_{j}^{2}} E\left[y^{\prime} X_{2} E X_{2}^{\prime} y\right] & =\frac{1}{v \sum z_{j}^{2}}\left[\underline{\alpha}^{\prime} M_{10}^{\prime} E M_{10} \underline{\alpha}\right]+\frac{1}{v \sum z_{j}^{2}} m_{\ell} \sum z_{j}^{2}(J-1) \sigma^{2} \\
& =\frac{1}{36}\left[\underline{\alpha}^{\prime} M_{10}^{\prime} E M_{10} \underline{\alpha}\right]+\sigma^{2}
\end{aligned}
$$




$$
E\left(C_{B}\right)=f_{8}(\beta)+\sigma^{2}
$$

onde $E_{0}(\beta)=\frac{1}{v \Sigma z_{j}^{2}}\left[\underline{\alpha}^{\prime} M_{10} E M_{10} \underline{\alpha}\right]=\frac{1}{36}\left[\underline{\alpha}^{\prime} M_{10}^{\prime} E M_{10} \underline{a}\right]$.

$$
4.5 .9 \text { - Esperiunça matenätica de } \frac{1}{v \sum x_{j}^{2}}\left(P_{3}-P_{1}\right)^{2}
$$

Como:

$$
P^{\prime} M_{19}^{-1} P=\frac{1}{v \Sigma z_{j}^{2}}\left[P_{1}-2 P_{2}+P_{3}\right]^{2}+\frac{1}{v \Sigma x_{j}^{2}}\left(P_{3}-P_{1}\right)^{2}
$$

entio:

$$
\begin{gathered}
\frac{1}{v \sum x_{j}^{2}}\left(P_{3}-P_{1}\right)^{2}=P^{\prime} M_{19}^{1} P-\frac{1}{v \sum z_{j}^{2}}\left(P_{1}-2 P_{2}+P_{3}\right)^{2} \\
=P^{\prime} M_{19}^{-1} P-C_{B} \\
\frac{1}{v \sum x_{j}^{2}} B\left[\left(P_{3}-P_{1}\right)^{2}\right]=E\left[P^{\prime} M_{19}^{-1} P\right]-E\left(C_{B}\right),
\end{gathered}
$$

que, por (4.5.4.c) e (4.5.8.a), fica:

$$
\begin{aligned}
= & \underline{b}^{\prime} M_{10} M_{19}^{-1} M_{10}^{\prime} \underline{b}+\underline{\alpha}^{\prime} M_{19} \underline{\alpha}+(J-1) \sigma^{2}- \\
& -\frac{1}{v \sum z_{j}^{2}}\left[\alpha^{\prime} M_{10}^{\prime} E M_{10} \underline{\alpha}\right]-\sigma^{2}
\end{aligned}
$$

Portanto:

$$
\frac{1}{v \sum x_{j}^{2}} E\left[\left(P_{3}-P_{1}\right)^{2}\right]=f_{9}(\beta)+(J-2) \sigma^{2}
$$


onde $f_{9}(\beta)=\underline{b}^{\prime} M_{10} M_{19}^{-1} M_{10}^{\prime} \stackrel{b}{b}+\underline{\alpha}^{\prime} M_{19} \underset{\alpha}{\alpha-}-\frac{1}{v \sum z_{j}^{2}}\left(\underline{\alpha}^{\prime} M_{10}^{\prime} E M_{10} \stackrel{\alpha}{\alpha}^{\prime}\right.$.

$$
\begin{aligned}
& \text { 4.5.10 - Esperança matemätica de } \frac{1}{\sum x_{j}^{2}}\left[y^{\prime} X_{6} X_{6}^{\prime} y\right] \\
& E\left\{\frac{1}{\sum x_{j}^{2}}\left[\sum\left(y_{i 3 k \ell}-y_{i 1 k \ell}\right)^{2}\right]\right\}=\frac{1}{\sum x_{j}^{2}} E\left[y^{\prime} X_{6} X_{6}^{\prime} y\right]
\end{aligned}
$$

que, por (4.5.1.b), fica:

$$
\begin{aligned}
& \frac{1}{\sum x_{j}^{2}} E\left[y^{\prime} X_{6} X_{6}^{\prime} y\right]=\frac{1}{\sum x_{j}^{2}}\left[\beta^{\prime} X^{\prime} X_{6} X_{6}^{\prime} X \beta+E\left(\dot{e}^{\prime} X_{6} X_{6}^{\prime} \varepsilon\right)\right] . \\
& \text { Sendo: } \\
& \mathrm{X}_{6}^{\prime} \mathrm{X}=\left[\begin{array}{llllll}
\phi & \phi & \phi & \phi & \mathrm{M}_{20}^{\prime} & \mathrm{M}_{21}
\end{array}\right] \\
& \widehat{B}=\left[\begin{array}{llllll}
\mathrm{m} & \underline{b}^{\prime} & \underline{v}^{\prime} & \underline{\tau}^{\prime} & \underline{\alpha}^{\prime} & \underline{c}^{\prime}
\end{array}\right] \\
& \frac{1}{\sum x_{j}^{2}}\left(\beta^{\prime} X^{\prime} X_{6} X_{6}^{\prime} X \beta\right)=\frac{1}{\sum x_{j}^{2}}\left(\underset{\sim}{\alpha^{\prime}} M_{20}+\underset{\sim}{c^{\prime}} M_{21}\right)\left(M_{20}^{\prime} \underset{\sim}{\alpha}+M_{21} \underset{\sim}{c}\right)
\end{aligned}
$$

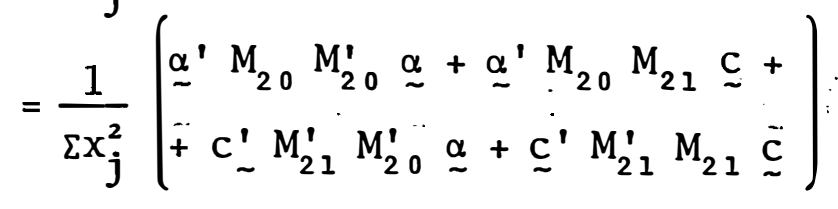

$$
\begin{aligned}
& =\frac{1}{\sum x_{j}^{2}} \underline{\alpha}^{\prime} M_{20} M_{20}^{\prime} \stackrel{\alpha}{\sim}+\frac{1}{2} \underline{c}^{\prime} M_{21}^{\prime} M_{21} \stackrel{c}{\sim} \\
& \frac{1}{\Sigma x_{j}^{2}} E\left[\varepsilon^{\prime} X_{6} X_{6}^{\prime} \varepsilon\right]=\frac{1}{\sum x_{j}^{2}} \sigma^{2} \quad \operatorname{tr}\left(X_{6} X_{6}^{\prime}\right) \\
& \operatorname{tr}\left(\mathrm{X}_{6} \mathrm{X}_{6}^{\prime}\right)=\operatorname{tr}\left(\mathrm{X}_{6}^{\prime} \mathrm{X}_{6}\right)=\sum \mathrm{X}_{\mathrm{j}}^{2} \mathrm{v}
\end{aligned}
$$


logo:

$$
\frac{1}{\sum x_{j}^{2}} E\left[\varepsilon^{\prime} X_{6} X_{6}^{\prime} \varepsilon\right]=\frac{1}{\sum x_{j}^{2}} \sigma^{2} \sum x_{j}^{2} v=v \sigma^{2}
$$

Portanto:

$$
\frac{1}{\sum x_{j}^{2}} E\left[y^{\prime} X_{6} X_{6}^{\prime} y\right]=f_{10}(\beta)+v \sigma^{2}
$$

onde: $f_{10}(\beta)=\frac{1}{\sum x_{j}^{2}}\left(\underline{\alpha}^{\prime} M_{20} M_{20}^{\prime} \stackrel{\alpha}{\underline{\alpha}}\right)+\frac{1}{\sum x_{j}^{2}}\left(\underline{c}^{\prime} M_{21}^{\prime} M_{21} \underset{\sim}{c}\right)$.

4:5:11 - Esperança matemática da soma de quadrados devida a efeito de blocos

De (4.4.2) temos:

SQ Blocos $=B^{\prime} M_{7}^{-1} B-\underline{\alpha}^{\prime} M_{10}^{\prime} M_{7}^{-1}, B$

onde: $\stackrel{\alpha}{\prime}^{\prime} M_{10}^{\prime} M_{7}^{-1}, B=C_{B}$

$$
\begin{gathered}
\text { SQ Blocos }=B^{\prime} M_{7}^{-1} B-C_{B} \\
E[S Q \text { Blocos }]=E\left[B^{\prime} M_{7}^{-1} B\right]-E\left[C_{B}\right]
\end{gathered}
$$

que, por $(4.5 .1 . d)$ e $(4.5 .8 . a)$, resultou:

$$
\begin{aligned}
E[S Q \text { Blocos }]= & \underline{\sim}^{\prime} M_{7} \underset{b}{b}+\underline{\alpha}^{\prime} M_{10}^{\prime} M_{7}^{-1} M_{10} \stackrel{\alpha}{\sim}+L \sigma^{2}- \\
& -\frac{1}{v \Sigma z_{j}^{2}}\left[\underline{\alpha}^{\prime} M_{10}^{1} E M_{10} \stackrel{\alpha}{\alpha}\right]-\sigma^{2}
\end{aligned}
$$


98.

Sendo:

$$
\stackrel{\alpha}{ }^{\prime} M_{10}^{\prime} M_{7}^{-1} M_{10} \stackrel{\alpha}{\alpha}=\frac{1}{v \sum z_{j}^{2}}\left[\underset{\sim}{\alpha} M_{10}^{\prime} E M_{10} \stackrel{\alpha}{\alpha}\right]
$$

obtivemus:

$$
\mathrm{E}[\mathrm{SQ} \text { Blocos }]=\mathrm{f}_{11}(\beta)+(\mathrm{L}-1) \sigma^{2}
$$

onde: $E_{11}(\beta)=\underline{\sim}^{\prime} M_{7} \underline{b}$.

4.5.12 - Esperança matemática da soma de quadrados devida a efeitos de vacas ignorando tratamentos.

$$
\begin{gathered}
\text { SQ Vacas (ign.trat.) }=\mathrm{V}^{\prime} \mathrm{M}_{12}^{-1} \mathrm{~V}-\frac{\mathrm{G}^{2}}{\mathrm{~N}} \\
\mathrm{E}[\mathrm{SQ} \text { Vacas (ign.trat.) }]=\mathrm{E}\left[\mathrm{V}^{\prime} \mathrm{M}_{12}^{-1} \mathrm{~V}\right]-\frac{1}{\mathrm{~N}} \mathrm{E}\left(\mathrm{G}^{2}\right)
\end{gathered}
$$

que, por $(4.5 .2 . c) \cdot$ e $(4.5 .7 . b)$, fica:

$$
\begin{aligned}
\mathrm{E}[\mathrm{SQ} \text { Vacas (ign.trat.) }]= & \mathrm{N} \mathrm{m}^{2}+{\underset{\sim}{\mathrm{v}}}^{\prime} \mathrm{M}_{12} \underset{\sim}{\mathrm{v}}+\underset{\sim}{2 v} \mathrm{M}_{13} \underset{\sim}{\tau}+ \\
& +\underset{\sim}{\tau} \mathrm{M}_{13}^{\prime} \mathrm{M}_{12}^{-1} \mathrm{M}_{13} \underset{\sim}{\tau}+ \\
& +\mathrm{v} \sigma^{2}-\mathrm{N} \mathrm{m}^{2}-\sigma^{2}
\end{aligned}
$$

Portanto:

$$
E[S Q \text { Vacas (ign.trat.) }]=f_{12}(\beta)+(v-1) \sigma^{2},
$$

onde: $f_{12}(\beta)=\underline{v}^{\prime} M_{12} \underline{v}+2 \underline{v}^{\prime} M_{13} \underline{\tau}+\underline{\tau}^{\prime} M_{13}^{\prime} M_{12}^{-1} M_{13} \tau$. 
4.5.13 - Esperança matenática da soma de quadrados devida a efeitos de tratamentos (ajustada)

Por (4.4.4), temos:

$$
\begin{aligned}
\text { SQ Trat. }(a j \cdot) & =\hat{\tau}^{\prime} Q^{*} \\
E[S Q \text { Trat. (aj.) }] & =E\left(\hat{\tau}^{\prime} Q^{*}\right)
\end{aligned}
$$

que, por $(4 \cdot 5.3 . e)$, fica:

$$
E[S Q \text { Trat. }(a j .)]=f_{13}(\beta)+(p-1) \sigma^{2}
$$

onde $f_{13}(\beta)=\tau^{\prime} Q^{*}$.

4.5.14 - Esperança matemätica da soma de quadrados devida a efeitos de períodos

Por (4.4.5):

$$
\begin{aligned}
\frac{1}{v} \Sigma P_{j}^{2}-\frac{G^{2}}{N} & =P^{\prime} M_{19}^{-1} P \\
E\left[\frac{1}{v} \Sigma P_{j}^{2}-\frac{G^{2}}{N}\right] & =E\left[P^{\prime} M_{19}^{-1} P\right]
\end{aligned}
$$

que, por $(4 \cdot 5.4 . c)$, fica:

$$
E\left[P^{\prime} M_{19}^{-1} P\right]=f_{14}(\beta)+(J-1) \sigma^{2},
$$

onde, $f_{14}(\beta)=\underline{b}^{\prime} M_{10} M_{19}^{-1} M_{10} \underset{\sim}{b}$ if $\underline{\alpha}^{\prime} M_{19} \stackrel{\alpha}{\alpha}$. 


\subsubsection{5 - Esperança matemática da soma de quadrados devida a} efeitos da interação período (linear) $x$ vacas

Por (4.4.6):

$S Q(c)=\frac{1}{\sum x_{j}^{2}} \sum_{i}\left(y_{i 3 k \ell}-y_{i 1 k \ell}\right)^{2}-\frac{1}{v \sum x_{j}^{2}}\left(P_{3}-P_{1}\right)^{2}$

Entāo:

que, por (4.5.10.a) e (4.5.9.a), temos:

$E[S Q(c)]=\frac{1}{\sum x_{j}^{2}} \stackrel{\alpha}{\prime}^{\prime} M_{20} M_{20}^{\prime} \stackrel{\alpha}{\sim}+\frac{1}{\sum x_{j}^{2}} \stackrel{c}{\sim}^{\prime} M_{21}^{\prime} M_{21} c+v \sigma^{2}-$

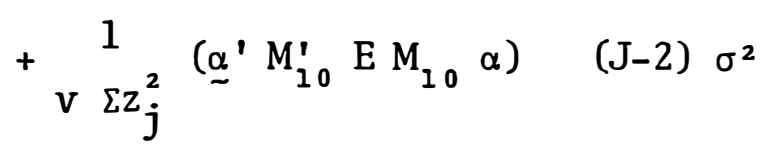

Sendo:

$$
\begin{aligned}
& \underline{\alpha}^{\prime} M_{20} M_{20}^{\prime} \underset{\sim}{\alpha}=v\left(\sum x_{j}^{2}\right)^{2} \alpha_{1}^{2} \\
& \alpha^{\prime} M_{19} \stackrel{\alpha}{\sim}=v \sum x_{j}^{2} \alpha_{1}^{2}+v \sum z_{j}^{2} \alpha_{2}^{2} \\
& 1 \\
& v \sum z_{j}^{2} \alpha^{\prime} M_{10}^{1} E M_{10} \alpha=v \sum z_{j}^{2} \alpha_{2}^{2}
\end{aligned}
$$




$$
\begin{aligned}
& \underline{\alpha}^{\prime} M_{20} M_{21} \underline{c}=\underline{c}^{\prime} M_{21} M_{20}^{\prime} \stackrel{\alpha}{\sim}=\phi \\
& \therefore \mathrm{E}[\mathrm{SQ}(\mathrm{C})]=\mathrm{f}_{-15}(\beta)+(\mathrm{V}=\mathrm{J}+2) \sigma^{2} \\
& \text { onde } f_{15}(\beta)=\frac{1}{\Sigma x_{j}^{2}} \stackrel{c}{\prime}^{\prime} M_{21}^{\prime} M_{21} \stackrel{c}{-} b^{\prime} M_{10} M_{19}^{-1} M_{10}^{\prime} \stackrel{b}{\sim} \cdot \\
& \text { 4.5.16 - Esperança matemătica da soma de quadridos do resí- } \\
& \text { duo } \\
& \text { Por (4.4), temos: } \\
& \mathrm{SQR}=\hat{y}^{\prime} \mathrm{y}-\hat{\beta^{\prime}} X^{\prime} y \\
& E[S Q R]=E\left[y^{\prime} y\right]-E\left[\hat{\beta}^{\prime} X^{\prime} Y\right] \\
& \mathrm{E}[\mathrm{SQR}]=\mathrm{E}\left[\mathrm{y}^{\prime} \mathrm{y}\right]-\mathrm{E}\left[\mathrm{G}^{2} / \mathrm{N}\right]-\mathrm{E}[\mathrm{SQB}]-\mathrm{E}[\mathrm{SQV}(\text { ign.trat. })]- \\
& \text { - E[SQ Trat. (aj.)] - E[SQP] - E[SQ(c)] ,. } \\
& \text { que, por }(4.5 .6),(4.5 .7),(4.5 .11),(4.5 .12),(4.5 .13),(4.5 .14) \mathrm{e} \\
& \text { (4.5.15), fica: }
\end{aligned}
$$




$$
\begin{aligned}
& \mathrm{E}[\mathrm{SQR}]=N \mathrm{~m}^{2}+\underline{b}^{\prime} \mathrm{M}_{7} \underset{\sim}{\mathrm{b}}+\underline{\sim}^{\prime} \mathrm{M}_{12} \underset{\sim}{\mathrm{v}}+\underline{\sim}^{\prime} \mathrm{M}_{16} \underset{\sim}{\mathrm{\tau}}+\underline{\alpha}^{\prime} \mathrm{M}_{19} \underset{\sim}{\alpha}+ \\
& +\underline{c}^{\prime} M_{21} \underline{c}+2 \underline{v}^{\prime} M_{13} \underline{\tau}+2 \underline{b}^{\prime} M_{10} \stackrel{\alpha}{\sim}+2 \underline{c}^{\prime} M_{20} \stackrel{\alpha}{\sim}+ \\
& +N \sigma^{2}-N m^{2}-\sigma^{2}-\underline{b}^{\prime} M_{7} \underline{b}-(L-1) \sigma^{2}- \\
& -\underline{v}^{\prime} M_{12} \underline{v}-2 \underline{\sim}^{\prime} M_{13} \underline{\tau}-\underline{\tau}^{\prime} M_{13}^{\prime} M_{12}^{-1} M_{13} \underline{\tau}-(v-1) \sigma^{2}- \\
& -\underline{\tau}^{\prime} Q^{*}-(p-1) \sigma^{2}-\underline{b}^{\prime} M_{10} M_{19}^{-1} M_{10}^{\prime} \underline{b}- \\
& -\stackrel{\alpha}{\prime}^{\prime} M_{19} \stackrel{\alpha}{\sim}-(J-1) \sigma^{2}-\frac{1}{2} \stackrel{c}{c}^{\prime} M_{21}^{\prime} M_{31} \underline{c}+ \\
& +\underset{\sim}{b} M_{10} M_{19}^{-1} M_{10}^{\prime} \underset{\sim}{b}-(v-J+2) \sigma^{2}
\end{aligned}
$$

Sendo:

$$
\frac{1}{2} \underline{c}^{\prime} M_{21}^{\prime} M_{21} \stackrel{c}{c}=\frac{1}{2} 2 \underline{c}^{\prime} M_{21} \stackrel{c}{c}=\stackrel{c}{c}^{\prime} M_{21} \underset{\sim}{c}
$$

e

$$
\begin{aligned}
\tau^{\prime} Q^{*}=\tau_{\sim}^{\prime} C^{*} \tau & =\underline{\tau}^{\prime}\left(M_{16}-M_{13}^{\prime} M_{12}^{-1} M_{13}\right) I= \\
& =\underline{\tau}^{\prime} M_{16} \underline{\tau}-\tau^{\prime} M_{13}^{\prime} M_{12}^{-1} M_{13} \tau
\end{aligned}
$$

logo:

$$
E[S Q R]=[N-L-2 v-p-J+4] \sigma^{2},
$$

sendo $J=3$,

$$
E[S Q R]=[N-L-2 v-p+1] \sigma^{2}
$$


QUADRO DAS ESPERANÇAS MATEMATTICAS DAS SOMAS DE QUADRADOS

\begin{tabular}{lc}
\hline Causas de Variação & $\begin{array}{c}\text { Esperança Matemática das } \\
\text { Somas de Quadrados }\end{array}$ \\
\hline Blocos & $f_{11}(\beta)+(L-1) \sigma^{2}$ \\
Vacas & $f_{12}(\beta)+(v-1) \sigma^{2}$ \\
Tratamentos (aj.) & $f_{13}(\beta)+(p-1) \sigma^{2}$ \\
Períodos & $f_{14}(\beta)+(J-1) \sigma^{2}$ \\
Interação Período (linear) x Vacas & $f_{15}(\beta)+(v-J+2) \sigma^{2}$ \\
Resíduo & $(N-L-2 v-p+1) \sigma^{2}$ \\
\hline-
\end{tabular}

onde:

$$
\begin{aligned}
& f_{11}(\beta)=\underline{w}^{\prime} M_{7} \underline{b} \\
& f_{12}(\beta)=\underline{\sim}^{\prime} M_{12} \underset{\sim}{v}+2 \underline{\sim}^{\prime} M_{13} \underset{\sim}{\tau}+\underline{\sim}^{\prime} M_{13}^{\prime} M_{12}^{-1} M_{13} \underset{\sim}{\tau} \\
& f_{13}(\beta)=\underline{\tau}^{\prime} Q^{*} \\
& f_{14}(\beta)=\underline{\sim}^{\prime} M_{10} M_{19}^{-1} M_{10}^{\prime} \underset{\sim}{b}+\underline{\sim}^{\prime} M_{19} \stackrel{\alpha}{\sim} \\
& f_{15}(\beta)=\frac{1}{\Sigma x_{j}^{2}} \stackrel{c}{\sim}^{\prime} M_{21}^{\prime} M_{21} \subseteq-\underline{b}^{\prime} M_{10} M_{19}^{1} M_{10}^{\prime} \stackrel{b}{b}
\end{aligned}
$$


4.6 - Distribuição das Formas Quadráticas

Os três teoremas apresentados a seguir serão utilizados nas deduções a serem efetuadas:

TEOREMA 1 (GRAYBILL, 1961):

Se $Y \cap N\left(\mu, \sigma^{2} I\right)$, então:

$Y^{\prime} A_{i} Y / \sigma^{2} \cap x^{2}\left(n_{i}, \lambda_{i}\right)$,

isto é, $Y^{\prime} A_{i} Y / \sigma^{2}$ tem distribuição de qui-quadrado não cen tral, com $n_{i}$ graus de liberdade e parâmetro de não centralidade $\lambda_{i}=\mu^{\prime} A_{i} \mu / 2 \sigma^{2}$, se e somente se $A_{i}$ for uma imatriz idempotente de característica $n_{i}$.

TEOREMA 2 (SEARLE, 1971):

Se $Y \cap N(\mu, \Sigma)$, então as formas quadráticas, $Y^{\prime} A_{i} Y=Y^{\prime} A_{j} Y$, são independentes se e somente se $A_{i} \Sigma A_{j}=\phi$, ou, de modo equivalente, $\mathrm{A}_{j} \Sigma \mathrm{A}_{\mathbf{i}}=\phi$.

TEOREMA 3 (SEARLE, 1971):

Se as variáveis aleatórias $U_{i}$ e $U_{j}$ são independentes, onde $U_{i} \cap x^{2}\left(n_{i}, \lambda_{i}\right)$ e $U_{j} \cap x^{2} n_{j}$, então: 


$$
\frac{\mathrm{u}_{\mathrm{i}} / \mathrm{n}_{\mathrm{i}}}{\mathrm{u}_{\mathrm{j}} / \mathrm{n}_{\mathrm{j}}} \cap \mathrm{F}\left(\mathrm{n}_{\mathrm{i}}, \mathrm{n}_{\mathrm{j}}, \lambda_{\mathrm{i}}\right),
$$

isto é, $\frac{U_{i} / n_{i}}{U_{j} / n_{j}}$ tem distribuição de $F$ não central com $n_{i}$ e $n_{j}$ graus de liberdiale e parâmetro de não centralidade $\lambda_{i}$.

4.6.1 - Distribuição de $B^{\prime} M_{7}^{-1} B / \sigma^{2}$

De $(4.5 .1 . a)$, temos:

$$
B^{\prime} \cdot M_{7}^{-1} B=y^{\prime} X_{2} M_{7}^{-1} X_{2}^{\prime} y=y^{\prime} A_{1} y,
$$

onde $A_{1-}=X_{2} M_{7}^{-1} X_{2}^{1}$, logo:

$$
\begin{aligned}
& A_{1}^{2}=X_{2} M_{7}^{-1} X_{2}^{\prime} X_{2} M_{7}^{-1} X_{2}^{\prime}, \\
& \text { Sendo } X_{2}^{\prime} X_{2}=M_{7} \text {, então: } \\
& A_{1}^{2}=X_{2} M_{7}^{-1} X_{2}^{\prime}=A_{1},
\end{aligned}
$$

isto è, $A_{1}$ è uma matriz idempotente. Assim. sendo, segue-se que:

$$
r\left(A_{1}\right)=\operatorname{tr}\left(X_{2} M_{7}^{-1} X_{2}^{\prime}\right),
$$

que, por (4.5.1), ficou:

$$
r\left(A_{1}\right)=\operatorname{tr}\left(A_{1}\right)=L \quad(4 \cdot 6 \cdot 1 \cdot c)
$$


No modelo (3.2), temos:

$$
Y \cap N\left(X \beta, \sigma^{2} I\right)
$$

então,

$$
\mu^{\prime} A_{1} \mu=\beta^{\prime} X^{\prime} X_{2} M_{7}^{-1} X_{2}^{\prime} X \beta,
$$

que, por $(4.5 .1 . c)$, resultou:

$$
\begin{aligned}
\mu^{\prime} A_{1} \mu & =\underline{b}^{\prime} M_{7} \underset{\sim}{b}+\underline{\sim}^{\prime} M_{10} \underset{\alpha}{\alpha}+\underline{\alpha}^{\prime} M_{10}^{\prime} \underline{b}+ \\
& +\underline{\alpha}^{\prime} M_{10}^{\prime} M_{7}^{-1} M_{10} \stackrel{\alpha}{\sim} \\
& \text { Pelo teorema } 1, \text { temos: } \\
& B^{\prime} M_{7}^{-1} B / \sigma^{2} \cap \chi^{2}\left(n_{1}, \lambda_{1}\right),
\end{aligned}
$$

onde:

$$
\begin{aligned}
& \mathrm{n}_{1}=\mathrm{L} ; \\
& \lambda_{1}=\frac{1}{2 \sigma^{2}}\left(\underline{\sim}^{\prime} \mathrm{M}_{7} \underline{\mathrm{b}}+\underline{\alpha}^{\prime} \mathrm{M}_{10}^{\prime} \mathrm{M}_{7}^{-1} \mathrm{M}_{10} \underset{\sim}{\alpha}\right)=\frac{1}{2 \sigma^{2}} f_{1}(\beta) . \\
& 4.6 .2 \text { - Distribuição de } V^{\prime} M_{12}^{-1} V^{\prime} \sigma^{2} \\
& \quad \text { De }(4.5 .2 . a), \text { temos: } \\
& V^{\prime} M_{12}^{-1} V=y^{\prime} x_{3} M_{12}^{-1} X_{3}^{\prime} y=y^{\prime} A_{2} y, \quad(4.6 .2 . a
\end{aligned}
$$

onde $A_{2}=X_{3} M_{12}^{-1} X_{3}^{\prime}$.

\section{Então:}

$$
A_{2}^{2}=X_{3} \quad M_{12}^{-1} X_{3}^{\prime} X_{3} M_{12}^{-1} X_{3}^{\prime}
$$




$$
\begin{array}{ll}
\text { Sendo } & X_{3}^{\prime} X_{3}=M_{12}, \log 0: \\
& A_{2}^{2}=X_{3} M_{12}^{-1} X_{3}^{\prime}=A_{2},
\end{array}
$$

isto $\bar{e}, A_{2} \bar{e}$ uma matriz idempotente. Então:

$$
r\left(A_{2}\right)=\operatorname{tr}\left(X_{3} M_{12}^{-1} X_{3}^{\prime}\right),
$$

que, por (4.5.2), ficou:

$$
r\left(A_{2}\right)=\operatorname{tr}\left(A_{2}\right)=v
$$

Por $(4.6 .1 . d)$, segue-se que:

$$
\mu^{\prime} A_{2} \mu=\beta^{\prime} X^{\prime} X_{3} M_{12}^{-1} X_{3}^{\prime} X \beta,
$$

que, por (4.5.2.b), fica:

$$
\begin{aligned}
\mu^{\prime} \mathrm{A}_{2} \mu= & N \mathrm{~m}^{2}+\underline{v}^{\prime} \mathrm{M}_{12} \underset{\sim}{\mathrm{v}}+2 \underline{\sim}^{\prime} \mathrm{M}_{13} \underline{\tau}+ \\
& +\underline{\sim}^{\prime} \mathrm{M}_{13}^{\prime} \mathrm{M}_{12}^{-1} \mathrm{M}_{13} \underline{\tau} \\
& \text { Pelo Teorema } 1, \text { tem-se: } \\
& \mathrm{V}^{\prime} \mathrm{M}_{12}^{-1} \mathrm{~V} / \sigma^{2} \cap \mathrm{X}^{2}\left(\mathrm{n}_{2}, \lambda_{2}\right)
\end{aligned}
$$

onde :

$$
\begin{aligned}
n_{2} & =v \\
\lambda_{2} & =\frac{1}{2 \sigma^{2}}\left[N m^{2}+\underline{v}^{\prime} M_{12} \underline{v}+2 \underline{v}^{\prime} M_{13} \underline{\tau}+\underline{\tau}^{\prime} M_{13}^{\prime} M_{12}^{-1} M_{13} \underset{\tau}{\tau}\right] \\
& =\frac{1}{2 \sigma^{2}} f_{2}(\beta) .
\end{aligned}
$$




$$
\text { 4.6.3 - Distribuição de } \hat{\tau}^{\prime} Q * / \sigma^{2}
$$

De $(4.5 .3 . a)$, temos:

$$
\hat{\tau}^{\prime} Q^{*}=y^{\prime} X W^{\prime} N^{{ }^{*} 1} W X^{\prime} y=y^{\prime} A_{3} y,(4 \cdot 6.3 . a)
$$

onde $A_{3}=X W^{\prime} N^{\Sigma_{1}} W^{\prime}$, logo,

$$
A_{3}^{2}=X W^{\prime} N^{\star_{1}} W^{\prime} X^{\prime} X W^{\prime} N^{ \pm_{1}} W^{\prime} X^{\prime},
$$

que, por (4.5.3.c), ficou:

$$
\mathrm{A}_{3}^{2}=\mathrm{XW}^{\prime} \mathrm{N}^{\underline{*} 1} \mathrm{C}^{*} \mathrm{~N}^{\underline{*} 1} \mathrm{WX}^{\prime}
$$

Sendo $C^{*}=\left(N^{*}-A\right)$, então:

$A_{3}^{2}=X W^{\prime} N^{N_{1}}\left(N^{*}-A\right) N^{k_{1}} W X^{\prime}$

$A_{3}^{2}=X W^{\prime} N^{*_{1}} N^{*} N^{*_{1}} W X^{\prime}-X W^{\prime} N^{*_{1}} A N^{*_{1}} W X^{\prime}$

Na equação (VI), temos:

$$
\begin{aligned}
A \hat{\tau} & =\phi \\
\text { A N }^{\underline{*} 1} Q^{*} & =\phi \\
A N^{* 1} \mathrm{~W}^{\prime} \mathrm{Y} & =\phi
\end{aligned}
$$

Como y é o vetor das observações, cujos valores são diferentes de zero, temos:
$A N^{ \pm} 1$ W $X^{\prime}=\phi$, 
logo,

$$
A_{3}^{2}=X W^{\prime} N^{ \pm 1} W^{\prime}=A_{3}, \quad(4 \cdot 6 \cdot 3 \cdot b)
$$

isto $\bar{e}, A_{3} \bar{e}$ uma matriz idempotente. Assim sendo, segue-se que :

$$
r\left(A_{3}\right)=\operatorname{tr}\left(X W^{\prime} N^{ \pm 1} W X^{\prime}\right.
$$

que, por (4.5.3.d), fica:

$$
\operatorname{tr}\left(X W^{\prime} N^{\underline{*} 1} W^{\prime} X^{\prime}\right)=p-1 \quad(4 \cdot 6 \cdot 3 . c)
$$

Por $(4.6 .1 . d)$, segue-se que:

$$
\mu^{\prime} A_{3} \mu=\beta^{\prime} X^{\prime} X W^{\prime} N^{ \pm 1} W X^{\prime} X \beta
$$

que, por 4.5 .3 , fica:

$$
\mu^{\prime} A_{3} \mu=\tau^{\prime} Q^{*}
$$

\section{Pelo Teorema 1, temos:}

$$
\tilde{\tau}^{\prime} Q^{*} / \sigma^{2} \cap x^{2}\left(n_{3}, \lambda_{3}\right)
$$

onde :

$$
\begin{aligned}
& n_{3}=p-1 ; \\
& \lambda_{3}=\frac{1}{2 \sigma^{2}}\left[\tau^{\prime} Q^{*}\right]=\frac{1}{2 \sigma^{2}} f_{3}(\beta) .
\end{aligned}
$$


4.0 .4 - Distribuição de $\mathrm{P}^{\prime} \mathrm{M}_{19}^{-1} \mathrm{P} / \mathrm{\sigma}^{2}$

$$
\begin{aligned}
& \operatorname{De}(4.5 .4 . a): \\
& P^{\prime} M_{19}^{-1} P=y^{\prime} X_{5} M_{19}^{-1} X_{5}^{\prime} y=y^{\prime} A_{4} y, \quad(4.6 .4 . a)
\end{aligned}
$$

onde $A_{4}=X_{5} M_{19}^{-1} X_{5}^{\prime}$.

Então .

$$
\begin{aligned}
& A_{4}^{2}=X_{5} M_{19}^{-1} X_{5}^{\prime} X_{5} M_{19}^{-1} X_{5}^{\prime}, \\
& \text { Sendo : } X_{5}^{\prime} X_{5}=M_{19}, \text { logo: } \\
& A_{4}^{2}=X_{5} M_{19}^{-1} X_{5}^{\prime}=A_{4},
\end{aligned}
$$

isto é, $A_{4}$ è uma matriz idempotente. Assim sendo, segue-se que:

$$
r\left(A_{4}\right)=\operatorname{tr}\left(X_{5} M_{19}^{-1} X_{5}^{\prime}\right),
$$

que, por 4.5.4, fica:

$$
r\left(A_{4}\right)=\operatorname{tr}\left(A_{4}\right)=(J-1)
$$

$$
\text { Por }(4.6 .1 . d) \text {, segue-se que: }
$$

$$
\mu^{\prime} A_{4} \mu=\beta^{\prime} X^{\prime} X_{5} M_{19}^{-1} X_{5}^{\prime} X_{\beta}^{\prime},
$$

que, por 4.6.4, fica:

$$
\mu^{\prime} A_{4} \mu=\underline{b}^{\prime} M_{10} M_{19}^{-1} M_{10}^{\prime} \underline{b}+\underline{\alpha}^{\prime} M_{19} \underline{\alpha} \quad \text { (4.6.4.d) }
$$


Pelo Teorema 1, temos:

$$
P^{\prime} M_{19}^{-1} P / \sigma^{2} \cap x^{2}\left(n_{4}, \lambda_{4}\right), \quad \text { (t.0.t.c) }
$$

onde:

$$
\begin{aligned}
& \mathrm{n}_{4}=(J-1) \\
& \lambda_{4}=\frac{1}{2 \sigma^{2}}\left[\underline{b}^{\prime} M_{10} M_{19}^{-1} M_{10}^{\prime} \underline{b}+\underline{\alpha}^{\prime} M_{19} \underline{\alpha}\right]=\frac{1}{2 \sigma^{2}} f_{4}(\beta) .
\end{aligned}
$$

4.6 .5 - Distribuição de $\mathrm{C}^{\prime} \mathrm{M}_{21}^{-1} \mathrm{C} / \sigma^{2}$

De $(4.5 .5 . a)$, temos:

$$
C^{\prime} M_{21}^{-1} C=y^{\prime} X_{6} \quad M_{21}^{-1} X_{6}^{\prime} y=y^{\prime} A_{5} y,
$$

onde, $A_{5}=X_{6} M_{21}^{-1} X_{6}^{\prime}$.

\section{Então:}

$$
A_{5}^{2}=X_{6} \quad M_{21}^{-1} X_{6}^{1} X_{6} \quad M_{21}^{-1} \bar{X}_{6}^{1} .
$$

Sendo $\mathrm{X}_{6}^{\prime} \mathrm{X}_{6}=\mathrm{M}_{21}$, logo:

$$
A_{5}^{2}=X_{6} \quad M_{21}^{-1} X_{6}^{1}=A_{5},
$$

isto é, $A_{5}$ é uma matriz idempotente. Então:

$$
r\left(A_{5}\right)=\operatorname{tr}\left(X_{6} M_{2 I}^{-1} X_{6}^{i}\right),
$$

que, por (4.5.5), fica:

$$
r\left(A_{5}\right)=\operatorname{tr}\left(A_{5}\right)=v \quad .
$$


Por $(4.6 .1 . d)$ segue-se que:

$$
\mu^{\prime} A_{5} \mu=\beta^{\prime} X^{\prime} X_{6} M_{21}^{-1} X_{6}^{\prime} X \beta
$$

que, por 4.5.5, fica:

$$
\mu^{\prime} A_{5} \mu=\underline{\alpha}^{\prime} M_{20} M_{21}^{-1} M_{20}^{\prime} \underset{\alpha}{\alpha}+c^{\prime} M_{21} \subseteq(\text { t.0.5.d) }
$$

Pelo teorema. 1, temos:

$$
C^{\prime} M_{2 I}^{-1} C / \sigma^{2} \cap x^{2}\left(n_{5}, \lambda_{5}\right), \quad(\text { t.6.5.e })
$$

onde:

$$
\begin{aligned}
& \mathrm{n}_{5}=v \\
& \lambda_{5}=\frac{1}{2 \sigma^{2}}\left[\stackrel{\alpha}{\prime} M_{20} M_{21}^{-1} M_{20}^{\prime} \stackrel{\alpha}{\sim}+\underline{\sim}^{\prime} M_{21} \underset{c}{c}\right]=\frac{1}{2 \sigma^{2}} f_{5}^{-}(\beta) .
\end{aligned}
$$

$$
4.6 .6 \text { - Distribuição de } y^{\prime} y / \sigma^{2}
$$

Temos $y^{\prime} y=y^{\prime} I y=y^{\prime} A_{6} y$, onde $A_{6}=I_{N}$. Como $A_{6}$ é uma matriz idempotente, de característica $N$, então, pelo teorema 1 e por (4.5.6.a), temos:

$$
y^{\prime} y / \sigma^{2} \cap x^{2}\left(n_{6}, \lambda_{6}\right),
$$

onde:

$$
\mathrm{n}_{6}=\mathrm{N} ;
$$




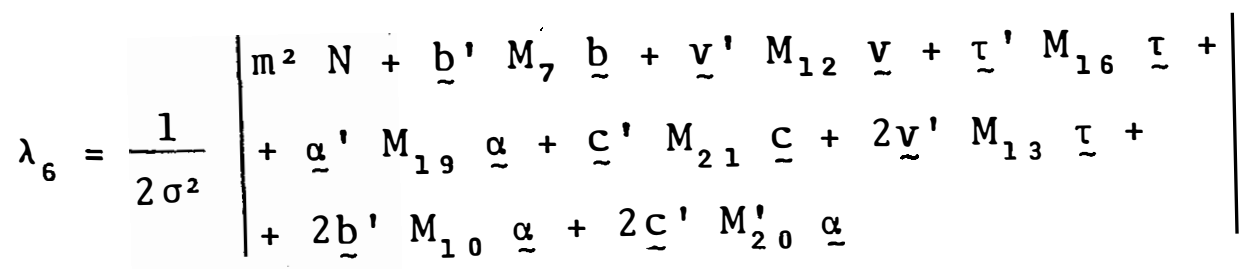

$$
\begin{aligned}
& =\frac{1}{2 \sigma^{2}} f_{6}(\beta) \text {. }
\end{aligned}
$$

4.6.7 - Distribuição da $\mathrm{SQR} / \sigma^{2}$

Por $(4.5 .16)$, temos:

$$
\begin{aligned}
S Q R= & y^{\prime} y-\frac{G^{2}}{N}-\left(B^{\prime} M_{7}^{-1} B-C_{B}\right)-\left(V^{\prime} M_{12}^{-1} V-\frac{G^{2}}{N}\right)- \\
& -\hat{\tau}^{\prime} Q^{*}-P^{\prime} M_{19}^{-1} P-\left[C^{\prime} M_{21}^{-1} C-\left(P^{\prime} M_{19}^{-1} P-C_{B}\right)\right] \\
S Q R= & y^{\prime} y-B^{\prime} M_{7}^{-1} B-V^{\prime} M_{12}^{-1} V-\hat{\tau}^{\prime} Q^{*}-C^{\prime} M_{21}^{-1} C \\
S Q R= & y^{\prime} y-y^{\prime} A_{1} y-y^{\prime} A_{2} y-y^{\prime} A_{3} y-y^{\prime} A_{5} y \\
S Q R= & y^{\prime}\left[I-A_{1}-A_{2}-A_{3}-A_{5}\right] y=y^{\prime} A_{7} y(4.6 .7 . a)
\end{aligned}
$$

onde: $A_{7}=I-A_{1}-A_{2}-A_{3}-A_{5}$.

\section{Então:}

$$
\begin{aligned}
A_{7}^{2}= & \left(I-A_{1}-A_{2}-A_{3}-A_{5}\right)\left(I-A_{1}-A_{2}-A_{3}-A_{5}\right) \\
A_{7}^{2}= & I^{2}-A_{1}-A_{2}-A_{3}-A_{5}-A_{1}+A_{1}^{2}+A_{1} A_{2}+A_{1} A_{3}+ \\
& +A_{1} A_{5}-A_{2}+A_{2} A_{1}+A_{2}^{2}+A_{2} A_{3}+A_{2} A_{5}-A_{3}+ \\
& +A_{3} A_{1}+A_{3} A_{2}+A_{3}^{2}+A_{3} A_{5}-A_{5}+A_{5} A_{1}+A_{5} A_{2}+ \\
& +A_{5} A_{3}+A_{5}^{2} .
\end{aligned}
$$


114

Já vimos que:

$$
A_{1}=A_{1}^{2} ; A_{2}=A_{2}^{2} ; A_{3}=A_{3}^{2} \text { e } A_{5}=A_{5}^{2}
$$

e sendo:

$$
\begin{aligned}
& A_{1} A_{2}=\left(A_{2} A_{1}\right)^{\prime}=\phi \\
& A_{1} A_{3}=\left(A_{3} A_{1}\right)^{\prime}=\phi \\
& A_{1} A_{5}=\left(A_{5} A_{1}\right)^{\prime}=\phi \\
& A_{2} A_{3}=\left(A_{3} A_{2}\right)^{\prime}=\phi \\
& A_{2} A_{5}=\left(A_{5} A_{2}\right)^{\prime}=\phi \\
& A_{3} A_{5}=\left(A_{5} A_{3}\right)^{\prime}=\phi
\end{aligned}
$$

então:

$$
A_{7}^{2}=I-A_{1}-A_{2}-A_{3}-A_{5}=A_{7} \quad(4 \cdot 6 \cdot 7 . b)
$$

isto é, A, é uma matriz idempotente. Então:

$$
\begin{aligned}
r\left(A_{7}\right)= & \operatorname{tr}\left(I_{N}-A_{1}-A_{2}-A_{3}-A_{5}\right), \\
r\left(A_{7}\right)= & \operatorname{tr}\left(I_{N}\right)-\operatorname{tr}\left(A_{1}\right)-\operatorname{tr}\left(A_{2}\right)- \\
& -\operatorname{tr}\left(A_{3}\right)-\operatorname{tr}\left(A_{5}\right) .
\end{aligned}
$$

Como $\operatorname{tr}\left(I_{N}\right)=N$, e us ando $(4.6 .1 . c),(4.6 .2 . c)$, $(4.6 .3 . c)$ e $(4.6 .5 . c)$, temos:

$$
\begin{aligned}
& r\left(A_{7}\right)=N-L-v-p+1-v \\
& r\left(A_{7}\right)=N-L-2 v-p+1
\end{aligned}
$$




$$
\begin{aligned}
\mu^{\prime} A_{7} \mu & =\beta^{\prime} X^{\prime}\left[I-A_{1}-A_{2}-A_{3}-A_{5}\right] X \beta \\
= & \beta^{\prime} X^{\prime} X \beta-\beta^{\prime} X^{\prime} A_{1} X \beta-\beta^{\prime} X^{\prime} A_{2} X \beta- \\
& -\beta^{\prime} X^{\prime} A_{3} X \beta-\beta^{\prime} X^{\prime} A_{5} X \beta \\
\cdot & 0 \\
\mu^{\prime} A_{7} \mu & 0 \\
\text { Pelo } & \text { teorema } 1, \text { temos: } \\
& S Q R / \sigma^{2} \cap X^{2}\left(n_{7}, 0\right)
\end{aligned}
$$

ou, de modo equivalente:

$$
\mathrm{SQR} / \sigma^{2} \cap \quad \mathrm{x}_{\mathrm{n}_{7}}^{2}
$$

onde, por $(4 \cdot 6 \cdot 7 . c): \mathrm{n}_{7}=\mathrm{N}-\mathrm{L}-2 \mathrm{v}-\mathrm{p}+1$. Tem, portan to, distribuição de qui-quadrado central, $\operatorname{com} n_{7}$ graus de liberdade.

4.7 - Distribuição dos Quocientes

$$
\begin{aligned}
& \text { 4.7.1 - Distribuição do quociente: } \frac{B^{\prime} M_{7}^{-1} B / n_{1} \sigma^{2}}{S Q R / n_{7} \sigma^{2}} \\
& \text { De }(4.6 .1 . a) \text { e }(4.6 .7 . a), \text { temos: } \\
& B^{\prime} M_{7}^{-1} B=y^{\prime} A_{1} y \\
& S Q R=y^{\prime}\left(I-A_{1}-A_{2}-A_{3}-A_{5}\right) y \cdot
\end{aligned}
$$


116.

$$
\begin{aligned}
A_{1}\left(1=A_{1}-A_{2}-A_{3}-A_{5}\right)= & A_{1}-A_{1}^{2}-A_{1} A_{2}- \\
& -A_{1} A_{3}-A_{1} A_{5} \\
= & A_{1}-A_{1}-\phi-\phi-\phi \\
= & \phi \quad(t .7 .1 . A)
\end{aligned}
$$

então, polu tourema 2 , com $\Sigma=\sigma^{2} \mathrm{I}$, conforme (4.6.1.d), $B^{\prime} M_{7}^{-1} B \Leftrightarrow S(? K$ silo independentes. Sendo assim, de $($ t.6.1. $\mathrm{f})$ e $(4.6 .7 .0)$, usimlo o teorema 3 , temos:

$$
\frac{B^{\prime} M_{7}^{-3} B / n_{1} \cdot \sigma^{2}}{S\left(S R / n_{7} \cdot \sigma^{2}\right.} \cap F\left(n_{1}, n_{7}, \lambda_{1}\right)
$$

onde:

$$
\begin{aligned}
& n_{3} \equiv l \\
& n_{1} \equiv N-l_{1}-2 v-p+1 \\
& \lambda_{1} \equiv \frac{1}{2 q^{2}} t_{3}(B)
\end{aligned}
$$

1.7 .2 - Distribuição do quociente $\frac{\mathrm{V}^{\prime} \mathrm{M}_{i 2}^{-1} \mathrm{~V} / \mathrm{n}_{2} \cdot \sigma^{2}}{\mathrm{SQR} / \mathrm{n}_{7} \cdot \sigma^{2}}$

De $(4.6 .2 . a)$ e $(4.6 .7 . a)$, temos:

$$
\begin{gathered}
V^{\prime} M_{12}^{-1} V=y^{\prime} A_{2} y \\
S Q R \approx y^{\prime}\left(I-A_{1}-A_{2}-A_{3}-A_{5}\right) y .
\end{gathered}
$$




\section{Como:}

$$
\begin{aligned}
A_{2}\left(I-A_{1}-A_{2}-A_{3}-A_{5}\right) & =A_{2}-A_{2} A_{1}-A_{2}^{2}-A_{2} A_{3}-A_{a} A_{5} \\
& =A_{2}-\phi-A_{2}-\phi-\phi \\
& =\phi \\
& (4.7 .3 . \therefore)
\end{aligned}
$$

então, pelo teorema $2, \operatorname{com} \Sigma=\sigma^{2} I$, conforme $(4.6 .1 .1)$, $V^{\prime} M_{12}^{-1} V$ e SQR são independentes. Sendo assim, de (4.0.2. $)$ e (4.6.7.e), usando o teorema 3 , temos:

$$
\frac{\mathrm{V}^{\prime} \mathrm{M}_{12}^{-1} \mathrm{~V} / \mathrm{n}_{2} \cdot \sigma^{2}}{\mathrm{SQR} / \mathrm{n}_{7} \cdot \sigma^{2}}
$$

onde:

$$
\begin{aligned}
& \mathrm{n}_{2}=\mathrm{v} \\
& \mathrm{n}_{7}=\mathrm{N}-\mathrm{L}-2 \mathrm{v}-\mathrm{p}+1 \\
& \lambda_{2}=\frac{1}{2 \sigma^{2}} \mathrm{f}_{2}(\beta) .
\end{aligned}
$$$$
4.7 .3 \text { - Distribuição do quociente } \frac{\hat{\tau}^{\prime} Q^{*}}{n_{3} \sigma^{2}} / \frac{S Q R}{n_{7} \sigma^{2}}
$$$$
\text { De }(4.6 .3 . a) \text { e }(4.6 .7 . a) \text {, temos: }
$$

$$
\hat{\tau}^{\prime} Q^{*}=y^{\prime} A_{3} Y
$$

$$
\text { SQRes. }=y^{\prime}\left(I-A_{1}-A_{2}-A_{3}-A_{5}\right) y
$$


118 .

Como :

$$
\begin{aligned}
A_{3}\left(I-A_{1}-A_{2}-A_{3}-A_{5}\right) & =A_{3}-A_{3} A_{1}-A_{3} A_{2}-A_{3}^{2}-A_{3} A_{5} \\
& =\phi \quad(4.7 .3 . a)
\end{aligned}
$$

então, pelo teorema 2 , com $\Sigma=\sigma^{2} \mathrm{I}$, conforme (4.6.1.d), $\hat{\tau}^{\prime} Q^{*}$ e SQR são independentes. Sendo assim, de (4.6.3.e) e (4.6.7.e), usando o teorema 3, temos:

$$
\frac{\hat{\tau}^{\prime} \mathrm{Q}^{*} / \mathrm{n}_{3} \cdot \sigma^{2}}{\mathrm{SQR} / \mathrm{n}_{7} \cdot \sigma^{2}} \cap \mathrm{F}\left(\mathrm{n}_{3}, \mathrm{n}_{7}, \lambda_{3}\right)
$$

onde :

$$
\begin{aligned}
& \mathrm{n}_{3}=\mathrm{p}-1 \\
& \mathrm{n}_{7}=\mathrm{n}-\mathrm{L}-2 \mathrm{v}-\mathrm{p}+1 \\
& \lambda_{3}=\frac{1}{2 \sigma^{2}} \mathrm{f}_{3}(\beta)
\end{aligned}
$$

4.7 .4 - Distribuição do quociente $\frac{\mathrm{P}^{\prime} \mathrm{M}_{19}^{-1} \mathrm{P} / \mathrm{n}_{4} \cdot \sigma^{2}}{\mathrm{SQR} / \mathrm{n}_{7} \cdot \sigma^{2}}$.

De (4.6.4.a) e (4.6.7.a), temos:

$$
P^{\prime} M_{19}^{-1} P=y^{\prime} A \quad y
$$

e

$$
S Q R=y^{\prime}\left(I-A_{1}-A_{2}-A_{3}-A_{5}\right) y
$$


119.

Como

$$
A_{4}\left(I-A_{1}-A_{2}-A_{3}-A_{5}\right) \neq \Phi,
$$

isto ê,o produto das matrizes não nulo, isso indica que $\mathrm{P}^{\prime} \mathrm{M}_{1}^{-1} \mathrm{P}$ e $\mathrm{SQR}$ não são independentes, logo o quociente:

$$
\frac{P^{\prime} M_{19}^{-1} P / n_{4} \cdot \sigma^{2}}{S Q R / n_{7} \cdot \sigma^{2}}
$$

não tem distribuição exata de F.

$$
\begin{aligned}
& \text { 4.7.5 - Distribuição do quociente } \frac{C^{\prime} M_{21}^{-1} C / n_{5} \cdot \sigma^{2}}{S Q R / n_{7} \cdot \sigma^{2}} \\
& \operatorname{De}(4.6 .5 . a) \text { e }(4 \cdot 6 \cdot 7 . a) \text {, temos: } \\
& C^{\prime} M_{21}^{-1} C=y^{\prime} A_{5} y \\
& \text { SQR }=\dot{y}^{\prime}\left(I-A_{1}-A_{2}-A_{3}-A_{5}\right) y
\end{aligned}
$$

Como

$$
\begin{aligned}
A_{5}\left(I-A_{1}-A_{2}-A_{3}-A_{5}\right) & =A_{5}^{2}-A_{5} A_{1}-A_{5} A_{2}-A_{5} A_{3}-A_{5} \\
& =\phi
\end{aligned}
$$

então, pelo teorema 2 , com $\Sigma=\sigma^{2} I$, conforme (4.6.1.d), $\mathrm{C}^{\prime} \mathrm{M}_{21}^{-1} \mathrm{C}$ e $\mathrm{SQR}$ são independentes. Sendo assim, de (4.6.5.e) e (4.6.7.e), usando o teorema 3 , temos: 


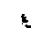

$$
\frac{\mathrm{C}^{\prime} \mathrm{M}^{-1} \mathrm{C} C / \mathrm{n}_{5} \cdot \sigma^{2}}{\mathrm{SQR} / \mathrm{n}_{7} \cdot \sigma^{2}} \quad F\left(\mathrm{n}_{5}, \mathrm{n}_{7}, \lambda_{5}\right)
$$

onde:

$$
\begin{aligned}
& \mathrm{n}_{5}=\mathrm{v} \\
& \mathrm{n}_{7}=\mathrm{N}-\mathrm{L}-2 v-p+1 \\
& \lambda_{5}=\frac{1}{2 \sigma^{2}} \mathrm{f}_{5}(\beta)
\end{aligned}
$$

4.8 - Testes de Hipóteses e Quadro da Análise da Variậncia

Nos ensaios em "switchback" existe geralmen: te interesse em se testar a hipótese básica:

$$
H_{o}: t_{k}=0 ; k=1,2, \ldots, p
$$

que é a hipótese de nulidade para efeitos de tratamentos. No entanto, outros testes de hipóteses podem eventualmente ser efetuados, conforme demonstrado em 4.7 e também sugerido no quadro a seguir. Entretanto, esse quadro deve ser analisado com cautela, pois vimos em 4.7.4 que Períodos e o Resíduo não são independentes, o que tor na o teste $F$ apenas aproximado para este caso. 
121.

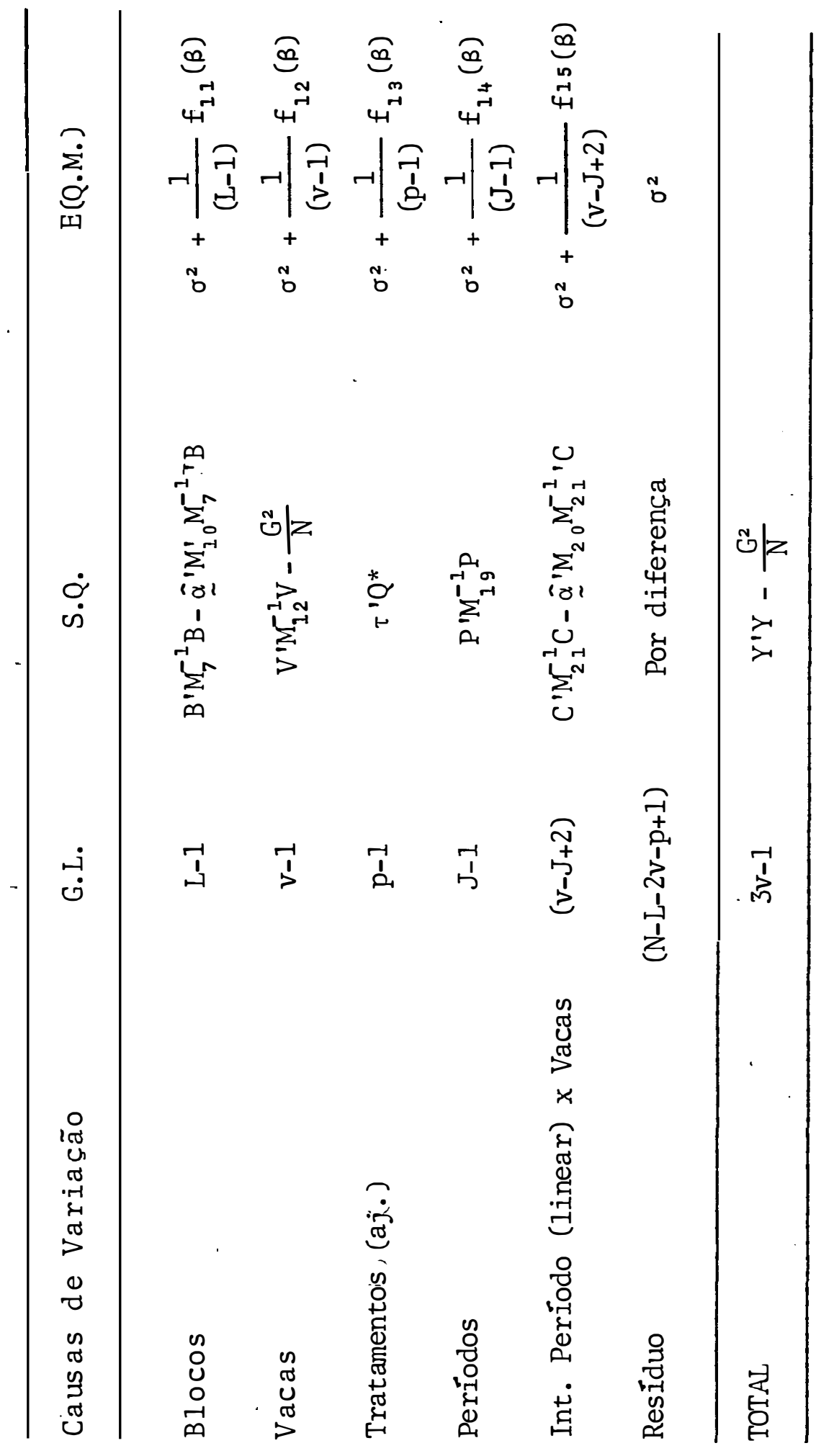


Assim, ficou detenninado o critério para o teste:

\begin{tabular}{ccc}
\hline HIPOTESE & G.L. & $\mathrm{F}_{\text {(observado) }}$ \\
\hline $\mathrm{H}_{\mathrm{o}}: \mathrm{t}_{\mathrm{k}}=0$ & $(\mathrm{p}-1) ;(\mathrm{N}-\mathrm{L}-2 \mathrm{v}-\mathrm{p}+1)$ & $\mathrm{QM}$ Trat (aj.)/QM Res. \\
\hline
\end{tabular}

4.9 - Comparações Múltiplas

Para se comparar as médias de tratamentos, consideramos o critério de Tukey, descrito em. NETER e WASSERMAN (1977), para modelos com estrutura de: erros $I \sigma^{2}$,

$$
\text { D.M.S. }=q \sqrt{(1 / 2) \bar{V}\left(\bar{Y}_{0}^{)}\right.} \text {, }
$$

onde D.M.S. é a diferença mínima significativa, q è o valor da amplitude total estudentizada e $\nabla\left(\gamma_{0}\right)$ é a variância estimada do contraste estimado $\mathbb{P}_{0}$, obtido por:

$$
P_{0}=\left(\hat{m}+\hat{t}_{k}\right)-\left(\hat{m}+\hat{t}_{k}^{\prime}\right)=\hat{t}_{k}-\hat{t}_{k^{\prime}}^{\prime}
$$

e

$$
\nabla\left(\hat{Y}_{0}\right)=\nabla\left(\hat{t}_{k}-\hat{t}_{k}\right)
$$


123.

5. ILUSTRAÇÃO DO METODO PROPOSTO

Os dados descritos em (3) foram utilizados para ilustrar a metodologia proposta.

5.1 - Modelo Matemátiço

Para se verificar a adequacidade do modelo adotado, foi feita a análise gráfica de resíduos. Para o modelo linear adotado escrito na for

$\mathrm{ma}$

$$
Y=X \beta+\varepsilon,
$$

onde: Y é o vetor das observações;

$\mathrm{X}$ é a matriz do delineamento;

B é o vetor dos parâmetros; 
$\varepsilon$ é um vetor de erros com distribuição normal de mé dia zero e variância $\sigma^{2} I$, independentes.

0 vetor (e) dos resíduos observados, onde os parâmetros são estimados pelo método dos mínimos quadrados, é obtido através de:

$$
\mathrm{e}=(Y-X \hat{\beta})^{\cdot},
$$

onde $\hat{\beta}=\left(X^{\prime} X\right)^{-1} X^{\prime} Y$, pois com as restrições admitidas em (4), a matriz X pasa a ter posto completo.

Para se verificar a adequacidade do modelo, segundo ANSCOMBE (1973), os resíduos e ${ }_{i}^{\prime} s$ quando plotados contra o valor ajustado $\widehat{P}_{i}$ não devem exibir nenhuma tendência em torno de seu valor médio $\bar{e}_{i}=0$. DRAPER e.:SMITH (1966) utilizam para a verificação dessa tendência o resíduo normalizado

$$
d_{i}=\frac{e_{i}}{\sqrt{Q M \operatorname{Res}}}
$$

Para o modelo ser considerado adequado, eles deverão distribuir-se aleatoriamente na forma de uma faixa horizontal em torno de zero.

Com os valores das estimativas dos parâmetros contidos em $\hat{\beta}$ podemos determinar:

$$
\underset{\sim}{\mathrm{e}}=\left(Y_{\text {obs }}-X \hat{\beta}\right)=\left(Y_{\text {obs }}-\hat{Y}\right) \text {, }
$$


e, de posse do quadro de anâlise de variância foram deter minados os resíduos normalizados:

$$
d_{i}=\frac{e_{i}}{\sqrt{Q M \text { Res. }}}
$$

Os valores dos $\mathrm{d}_{\mathbf{i}}$ da Tabela 2 foram plotados contra os valores de $\mathbf{P}_{\mathbf{i}}$ (Figura 1 ), através da qual pudemos observar que o modelo se mostra adequado para a análi se dos dados. 
Tabela 2 - Valores obtidos para as estimativas de Y, do

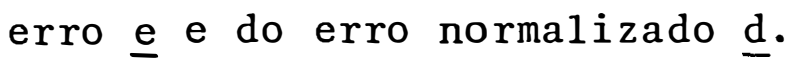

\begin{tabular}{|c|c|c|}
\hline $\begin{array}{c}\text { Y EST IMADO } \\
\bar{Y}_{\mathbf{i}}\end{array}$ & $\begin{array}{c}\text { ERRO } \\
e_{i}\end{array}$ & $\begin{array}{c}\text { ERRO } \\
\text { NORMALI ZADO } \\
\mathrm{d}_{\mathbf{i}} \\
\end{array}$ \\
\hline $\begin{array}{l}20,34 \\
20,59 \\
17,82 \\
17,66 \\
18,28 \\
15,43 \\
25,82 \\
25,47 \\
22,85 \\
15,00 \\
14,57 \\
11,05 \\
19,96 \\
17,87 \\
13,96 \\
12,49 \\
13,44 \\
12,19 \\
13,93 \\
14,11 \\
11,59 \\
18,92 \\
18,89 \\
15,69 \\
15,96 \\
15,40 \\
12,90 \\
25,16 \\
23,97 \\
20,01 \\
19,08 \\
17,37 \\
14,15 \\
19,03 \\
17,30 \\
13,69\end{array}$ & $\begin{array}{r}-0,3 \\
0,6 \\
-0,3 \\
-0,36 \\
0,72 \\
-0,36 \\
-0,46 \\
0,92 \\
-0,46 \\
0,52 \\
-1,04 \\
0,52 \\
-0,03 \\
0,06 \\
-0,03 \\
0,63 \\
-1,26 \\
0,63 \\
0,15 \\
-0,31 \\
0,15 \\
0,12 \\
-0,24 \\
0,12 \\
0,23 \\
-0,46 \\
0,23 \\
-0,09 \\
0,18 \\
-0,09 \\
-0,13 \\
0,27 \\
-0,13 \\
-0,28 \\
0,56 \\
-0,28\end{array}$ & $\begin{array}{r}-0,3041 \\
0,6082 \\
-0,3041 \\
-0,3650 \\
0,7300 \\
-0,3650 \\
-0,4663 \\
0,9326 \\
-0,4663 \\
0,5272 \\
-1,0544 \\
0,5272 \\
-0,0304 \\
0,0608 \\
-0,0304 \\
0,6387 \\
-1,2774 \\
0,6387 \\
0,1521 \\
-0,3142 \\
0,1521 \\
0,1217 \\
-0,2434 \\
0,1217 \\
0,2332 \\
-0,4664 \\
0,2332 \\
-0,0912 \\
0,1824 \\
-0,0912 \\
-0,1318 \\
0,2736 \\
-0,1318 \\
-0,2839 \\
0,5678 \\
-0,2839\end{array}$ \\
\hline
\end{tabular}


127.

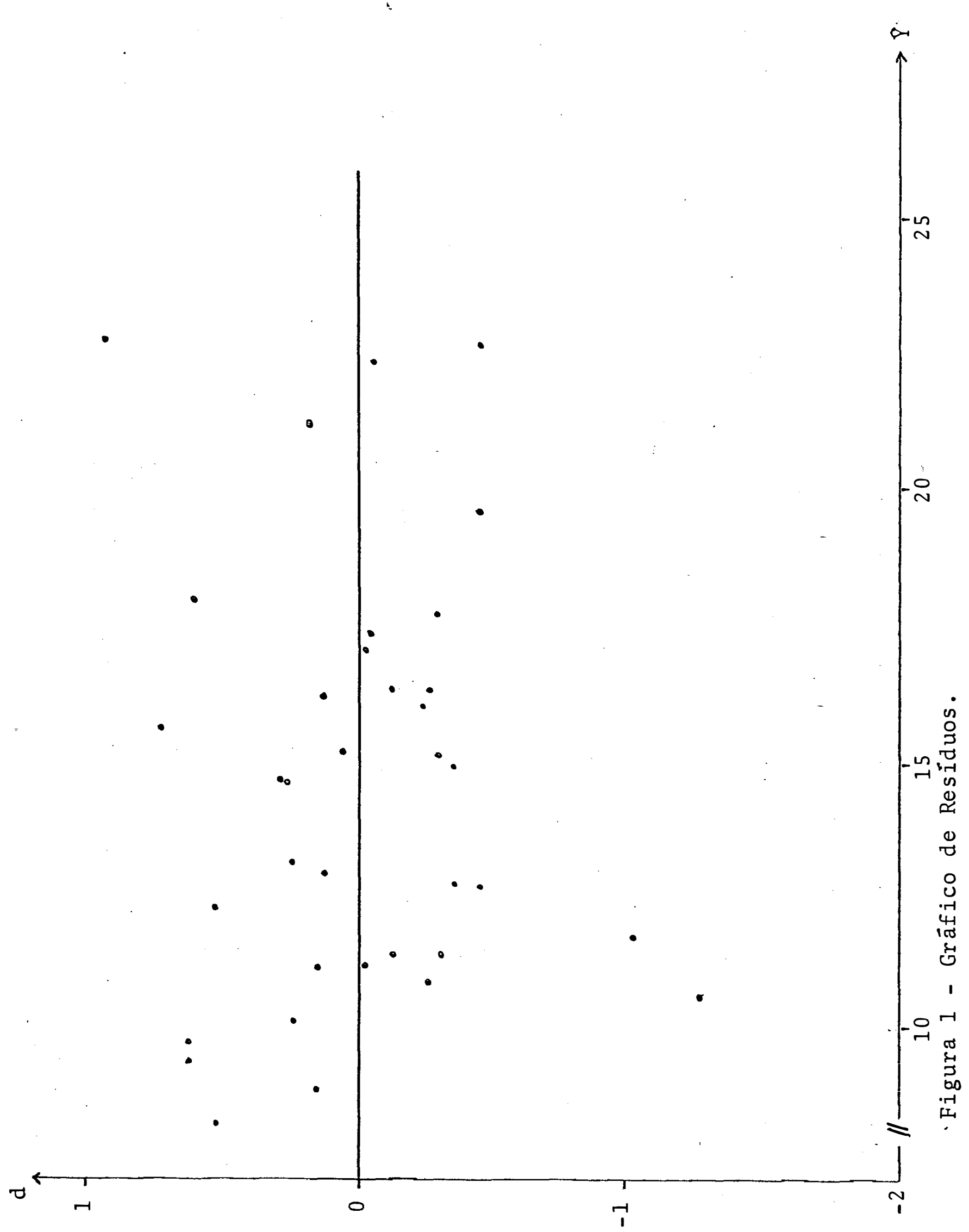




\section{2 - Equações Normais}

Verificadas as exigências do modelo matemâtico, determinou-se o sistema de equações normais, conforme (I):

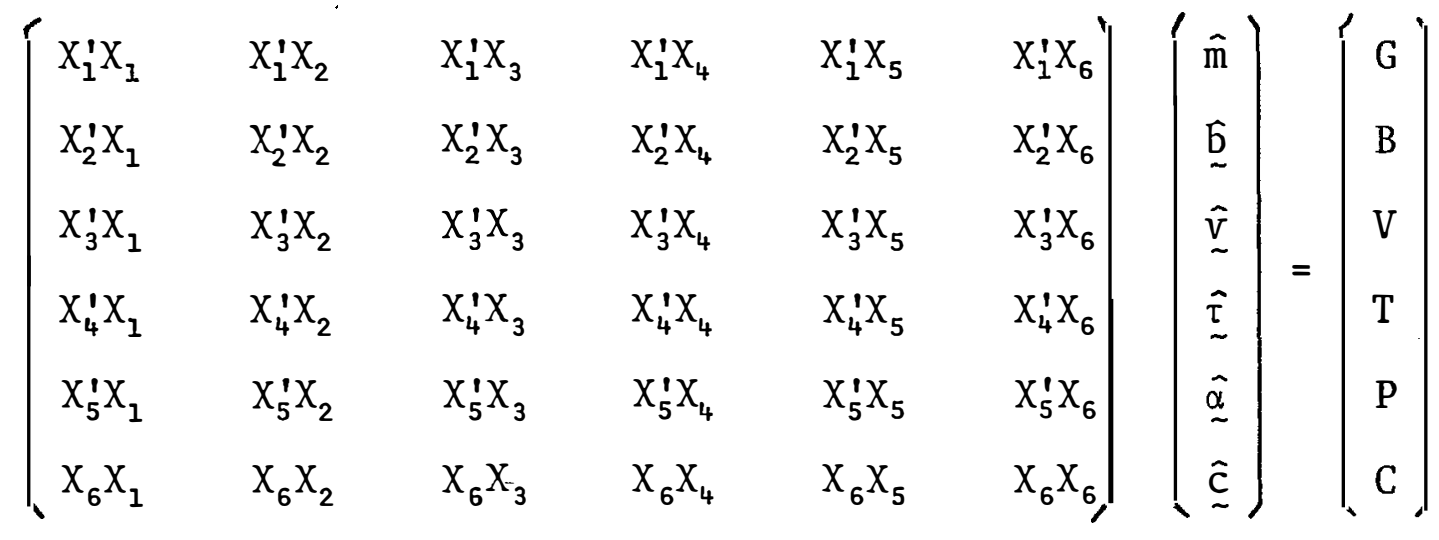

onde, para: $k=1,2, \ldots, p=3$ tratamentos,

$$
\begin{aligned}
& \mathrm{j}=1,2, \ldots, \mathrm{J}=3 \text { pexíodos, } \\
& \mathrm{s}=1,2, \ldots, \mathrm{S}=6 \text { sequências, } \\
& \mathrm{r}=1,2, \ldots, \mathrm{R}=2 \text { repetições de sequências, } \\
& \ell=1,2, \ldots, \mathrm{L}=2 \text { blocos, } \\
& \mathrm{i}=1,2, \ldots, \mathrm{V}=12 \text { animais, } \\
& \mathrm{X}_{1}^{\prime} \mathrm{X}_{1}=\mathrm{M}_{1}=\mathrm{N}=3 \mathrm{v}=36 \\
& \mathrm{X}_{1}^{\prime} \mathrm{X}_{2}=\mathrm{M}_{2}=(1)^{\phi}(2) .
\end{aligned}
$$




$$
\begin{aligned}
& X_{1}^{\prime} X_{3}=M_{3}=3(1)^{E}(12) \\
& X_{1}^{\prime} X_{4}=M_{4}=12_{(1)^{E}}^{E}(3) \\
& X_{1}^{\prime} X_{5}=M_{5}=(1)^{\phi}(2) \\
& X_{1}^{\prime} X_{6}=M_{6}=(1)^{\phi}(12) \\
& X_{2}^{\prime} X_{2}=M_{7}=36 I_{(2)} \\
& X_{2}^{\prime} X_{3}=M_{8}=(2)^{\phi}(12) \\
& X_{2}^{\prime} X_{4}=M_{9}=(2)^{\phi}(3) \\
& X_{2}^{\prime} X_{5}=M_{10}=6 \cdot\left(\begin{array}{ll}
0 \\
0
\end{array}\right. \\
& X_{2}^{\prime} X_{6}=M_{11}=(2)^{\phi}(12) \\
& X_{3}^{\prime}=M_{12}=3 I_{(12)}
\end{aligned}
$$


$X_{3}^{\prime} X_{4}=M_{13}=\left(\begin{array}{lll}2 & 1 & 0 \\ 2 & 0 & 1 \\ 1 & 2 & 0 \\ 0 & 2 & 1 \\ 1 & 0 & 2 \\ 0 & 1 & 2 \\ 2 & 1 & 0 \\ 2 & 0 & 1 \\ 1 & 2 & 0 \\ 0 & 2 & 1 \\ 1 & 0 & 2 \\ 0 & 1 & 2\end{array}\right)_{(3)}$

$$
\begin{aligned}
& X_{3}^{\prime} X_{5}=M_{14}=(12)^{\phi}(2) \\
& X_{3}^{\prime} X_{6}=M_{15}=\left(\begin{array}{ll}
12
\end{array}\right)^{\phi}(12) \\
& X_{4}^{\prime} X_{4}=M_{16}=12 I_{(3)} \\
& X_{4}^{1} X_{5}=M_{17}=(3)^{\phi}(2) \\
& X_{4}^{\prime} X_{6}=M_{18}=(3)^{\phi}\left(\begin{array}{ll}
12
\end{array}\right) \\
& X_{5}^{\prime} X_{5}=M_{19}=12\left(\begin{array}{ll}
2 & 0 \\
0 & 6
\end{array}\right]_{(2)}={ }_{(2)}\left(\begin{array}{rr}
24 & 0 \\
0 & 72
\end{array}\right)_{(2)}
\end{aligned}
$$




$$
X_{5}^{\prime} X_{6}=M_{20}=\left(\begin{array}{llllllllllll}
2 & 2 & 2 & 2 & 2 & 2 & 2 & 2 & 2 & 2 & 2 & 2 \\
0 & 0 & 0 & 0 & 0 & 0 & 0 & 0 & 0 & 0 & 0 & 0
\end{array}\right)_{(12)}
$$$$
X_{6}^{\prime} X_{6}=M_{21}=2 I_{(12)}
$$$$
b=\left[\begin{array}{c}
b_{1} \\
b_{2}
\end{array} \mid \quad \hat{v}=\left[\begin{array}{c}
\hat{v}_{1} \\
\hat{v}_{2} \\
\hat{v}_{3} \\
\ldots \\
\hat{v}_{12}
\end{array}\right] \quad \hat{\tau}=\left[\begin{array}{c}
\hat{t}_{1} \\
\hat{t}_{2} \\
\hat{t}_{3}
\end{array}\right]\right.
$$

$$
\hat{\alpha}=\left(\begin{array}{c}
\hat{\alpha}_{1} \\
\hat{\alpha}_{2}
\end{array}\right)=\hat{c}=\left(\begin{array}{c}
\hat{c}_{1} \\
\hat{c}_{2} \\
\hat{c}_{3} \\
\cdots \\
\hat{c}_{12}
\end{array}\right)
$$

$$
G=621,94
$$


132.

$$
\mathrm{B}=\left(\begin{array}{l}
-15,87 \\
-13,97
\end{array}\right) ; \quad \mathrm{P}=\left(\begin{array}{l}
223,35 \\
217,26 \\
181,33
\end{array}\right) ; \quad \mathrm{T}=\left(\begin{array}{l}
207,49 \\
214,18 \\
200,27
\end{array}\right)
$$

$$
V=\left(\begin{array}{l}
58,75 \\
51,38 \\
74,14 \\
40,62 \\
51,78 \\
38,12 \\
39,62 \\
53,50 \\
44,26 \\
69,14 \\
50,61 \\
50,02
\end{array}\right) \quad C=\left[\begin{array}{l}
-2,52 \\
-2,23 \\
-2,97 \\
-3,95 \\
-6,00 \\
-0,30 \\
-2,34 \\
-3,23 \\
-3,06 \\
-5,15 \\
-4,93 \\
-5,34
\end{array}\right)
$$


5.3 - Solução do Sistema de Equações Normais

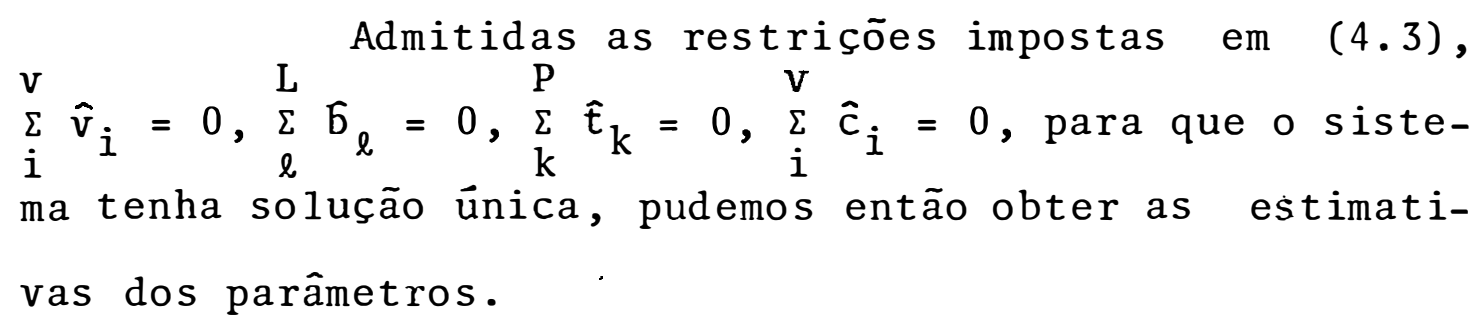

5.3 .1 - Estimativa da mēdia

Por (4.3.1) temos:

$$
\widehat{\mathrm{m}}=\mathrm{M}^{-1} \mathrm{G}
$$

sendo $M_{1}=36$ e $G=621,94$, obteve-se

$$
\hat{\mathrm{m}}=\frac{621,94}{36}=17,276111
$$

5.3.2 - Estimativa dos efeitos de tratamentos e1 minando-se efeitos de vacas

De (4.3.2) temos:

$$
\hat{\mathrm{T}}=\mathrm{N}^{*} \mathrm{Q}^{*}
$$

$$
\begin{gathered}
\text { e } N^{*}=\left(\dot{r}_{k}-\frac{\lambda_{1}}{J}+\frac{\lambda_{2}}{J}\right) I_{(p)}, \\
\text { sendo } \lambda_{1}=\sum_{i, k} a_{i, k}^{2} \operatorname{para} i=i^{\prime}, k=k^{\prime}, \\
\lambda_{2}=\sum_{i, k}\left(a_{i}^{\prime} k^{\prime} \cdot a_{i} k^{\prime}\right) \operatorname{para} i \neq i^{\prime}, k \neq k^{\prime}
\end{gathered}
$$


resulta

$$
N^{*}=\left(12-\frac{20}{3}+\frac{8}{3}\right) I_{(3)}=8 \cdot I_{(3)}
$$

portanto,

$$
\begin{gathered}
N^{ \pm 1}=\frac{1}{8} I_{(3)} \\
\text { A matriz } Q^{*} \text { ficou: } \\
Q^{*}=\left[\begin{array}{l}
T_{1}-\frac{2}{3}\left(V_{1}+V_{2}+V_{7}+V_{8}\right)-\frac{1}{3}\left(V_{3}+V_{5}+V_{9}+V_{11}\right) \\
T_{2}-\frac{2}{3}\left(V_{3}+V_{4}+V_{9}+V_{10}\right)-\frac{1}{3}\left(V_{1}+V_{6}+V_{7}+V_{12}\right) \\
T_{3}-\frac{2}{3}\left(V_{5}+V_{6}+V_{11}+V_{12}\right)-\frac{1}{3}\left(V_{2}+V_{4}+V_{8}+V_{10}\right)
\end{array}\right]
\end{gathered}
$$

onde, substituindo-se os valores de $T_{k}$ e $V_{i}$, resultou:

$$
Q^{*}=\left[\begin{array}{r}
-1,606667 \\
-0,096667 \\
1,703334
\end{array}\right] \text {, }
$$

portanto:

$$
\hat{\tau}=\left(\begin{array}{l}
\hat{\mathrm{t}}_{1} \\
\hat{\mathrm{t}}_{2} \\
\hat{\mathrm{t}}_{3}
\end{array}\right)=\left(\begin{array}{r}
-0,200833 \\
-0,012083 \\
0,212916
\end{array}\right) ; \sum_{\mathrm{k}=1}^{3} \hat{\mathrm{t}}_{\mathrm{k}}=0
$$


5.3.3 - Estimativa dos efeitos de vacas:

De acordo com (4.3.3), tem-se:

$$
\left.\begin{array}{l}
\hat{v}_{1} \\
\hat{v}_{2} \\
\hat{v}_{3} \\
\hat{v}_{4} \\
\hat{v}_{5} \\
\hat{v}_{6} \\
\hat{v}_{7} \\
\hat{v}_{8} \\
\hat{v}_{9} \\
\hat{v}_{10} \\
\hat{v}_{11} \\
\hat{v}_{12}
\end{array}\right]=\frac{1}{3}\left[\begin{array}{l}
v_{1}-3 \hat{m}-\left(2 \hat{t}_{1}+\hat{t}_{2}\right) \\
v_{2}-3 \hat{m}-\left(2 \hat{t}_{1}+\hat{t}_{3}\right) \\
v_{3}-3 \hat{m}-\left(\hat{t}_{1}+2 \hat{t}_{2}\right) \\
v_{4}-3 \hat{m}-\left(2 \hat{t}_{2}+\hat{t}_{3}\right) \\
v_{5}-3 \hat{m}-\left(\hat{t}_{1}+2 \hat{t}_{3}\right) \\
v_{6}-3 \hat{m}-\left(\hat{t}_{2}+2 \hat{t}_{3}\right) \\
v_{7}-3 \hat{m}-\left(2 \hat{t}_{1}+\hat{t}_{2}\right) \\
v_{8}-3 \hat{m}-\left(2 \hat{t}_{1}+\hat{t}_{3}\right) \\
v_{9}-3 \hat{m}-\left(\hat{t}_{1}+2 \hat{t}_{2}\right) \\
v_{10}-3 \hat{m}-\left(2 \hat{t}_{2}+\hat{t}_{3}\right) \\
v_{11}-3 \hat{m}-\left(\hat{t}_{1}+2 \hat{t}_{3}\right) \\
v_{12}-3 \hat{m}-\left(\hat{t}_{2}+2 \hat{t}_{3}\right)
\end{array}\right]
$$

então, 


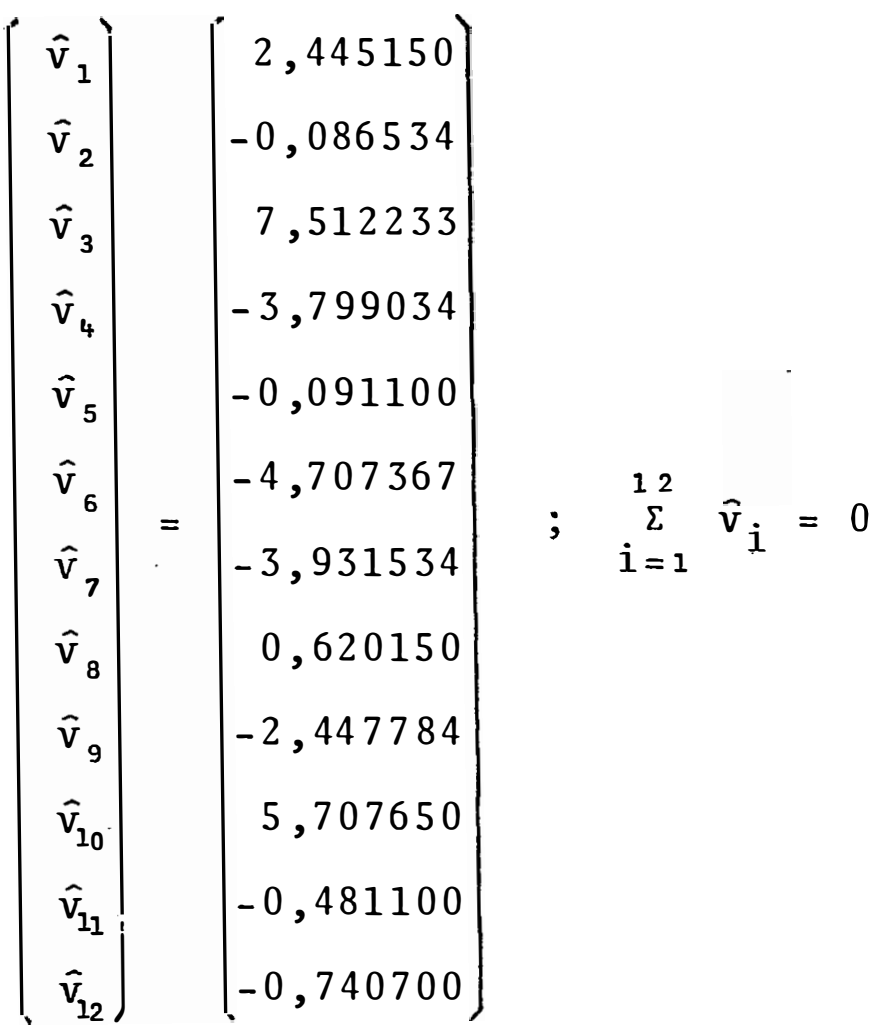

5.3.4 - Estimativa dos efeitos linear e quadrätico de períodos

De (4.3.4) temos:

$$
\begin{aligned}
& \hat{\alpha}_{1}=\frac{1}{12(2)}(181,33-223,35) \\
& \hat{\alpha}_{2}=\frac{1}{12(6)}(223,35-2.217,26+181,33)
\end{aligned}
$$

riesul tando em: 


$$
\left(\begin{array}{l}
\hat{\alpha}_{1} \\
\hat{\alpha}_{2}
\end{array}\right)=\left(\begin{array}{l}
-1,750833 \\
-0,414444
\end{array}\right)
$$

5.3.5 - Estimativa dos efeitos de blocos

De acordo com (4.3.5), as estimativas dos efeitos de blocos são obtidas através de:

$$
\sigma_{\ell}=\frac{1}{m_{\ell} \cdot \Sigma z_{j}^{2}} B_{\ell}-\hat{\alpha}_{2}
$$

ou

$$
\sigma_{\ell}=\frac{1}{m_{\ell} \cdot \Sigma z_{j}^{2}} B_{\ell}-\frac{1}{36}\left(P_{1}-2 P_{2}+P_{3}\right),
$$

resultando:

$$
\left(\begin{array}{c}
\mathrm{b}_{1} \\
\mathrm{~b}_{2}
\end{array}\right)=\left(\begin{array}{r}
-0,026389 \\
0,026389
\end{array}\right) ; \sum_{\ell=1}^{2} \mathrm{~b}_{\ell}=0 .
$$

5.3.6 - Estimativa dos efeitos da interação período (linear) $\mathrm{x}$ vacas

Por $(4.3 .6)$, obtemes: 
138 .

$$
\left[\begin{array}{l}
\hat{c}_{1} \\
\hat{c}_{2} \\
\hat{c}_{3} \\
\hat{c}_{4} \\
\hat{c}_{5} \\
\hat{c}_{6} \\
\hat{c}_{7} \\
\hat{c}_{8} \\
\hat{c}_{9} \\
\hat{c}_{10} \\
\hat{c}_{11} \\
\hat{c}_{12}
\end{array}\right]=\left[\begin{array}{r}
0,490833 \\
0,635833 \\
0,265833 \\
-0,224166 \\
-1,249166 \\
1,600833 \\
0,580833 \\
0,135833 \\
0,220833 \\
-0,824166 \\
-0,714166 \\
-0,919166
\end{array}\right], \quad \sum \hat{c}_{i}=0
$$

5.4 - Somas de Quadrados

5.4.1 - Obtenção da Soma de Quadrados de $\hat{m}$ :

De acordo com (4.4.1):

$$
\mathrm{SQ}(\hat{\mathrm{m}})=10.744,704540 \text {. }
$$

5.4.2 - Obtenção da Soma de Quadrados de Blocos:

Por (4.4.2) obtemos:

SQ B1ocos $=0,050138$. 
5.4.3 - Obtenção da Soma de Quadrados de Tratamentos (ajustada):

De (4.4.3) temos que:

SQ Trat.(aj.) $=\hat{\tau}^{\prime} Q^{*}$,

que para o exemplo em estudo resultou em:

$$
\begin{aligned}
\text { SQ Trat. (aj.) } & =Q_{k}^{*} N^{* 1} Q_{k}^{*} \\
& =\frac{1}{8} \sum_{k=1}^{3} Q_{k}^{*}
\end{aligned}
$$

SQ Trat.(aj.) $=0,686508$

5.4.4 - Obtenção da Soma de Quadrados de Vacas

Por (4.4.4), temos:

SQ Vacas (ign.trat.) $=457,678060$

5.4.5 - Obtenção da Soma de Quadrados de Períodos

Por (4.4.5) temos:

SQ Períodos $=85,937043$,

ond e ,

SQ Períodos (linear) $=73,570016$,

SQ Períodos (qudrātico) $=12,367027$. 
5.4.6 - Obtenção da Soma de Quadrados da Interação Período (linear) x Vacas

De (4.4.6) obtivemos:

SQ Interação (Período:1inear x Vacas) $=14,655883$.

5.5 - Quadro da Anālise de Variância e Testes de Hipóteses

Obtivemos o seguinte quadro para a análise de variância:

\begin{tabular}{|c|c|c|c|c|}
\hline Causas de Variação & G.L. & S.Q. & Q.M. & \\
\hline Blocos & 1 & 0,050138 & 0,050139 & \\
\hline Vacas (V) & 11 & 457,678060 & 41,607096 & \\
\hline Tratamentos (aj.) & 2 & 0,686508 & 0,343254 & $0,3528^{\mathrm{nS}}$ \\
\hline Períodos (P) & 2 & 85,937043 & 42,968522 & \\
\hline Interação $\mathrm{P}$ (linear) $\mathrm{xV}$ & 11 & 14,655883 & 1,332353 & $1,37^{\mathrm{nS}}$ \\
\hline Resíduo & 8 & 7,783626 & 0,972953 & \\
\hline Total & 35 & 566,791230 & & \\
\hline
\end{tabular}


que não forneceu evidências para a rejeição de $\mathrm{H}_{\mathrm{o}}$ • Concluimos,, então, pela não existência de efeito diferencial significativo entre tratamentos.

\section{6 - Comparações Múltiplas}

Devido à não rejeição de $\mathrm{H}_{\mathrm{o}}$, pela análise de variância, não ê necessário efetuar teștes de compara ções mültiplas.

Apenas como ilustração, foram colocados os valores das médias de tratamentos e a diferença mínima significativa do teste de. Tukey.

Médias de Tratamentos Ajustadas, em kg:

$$
\begin{aligned}
& \text { A B } \\
& 17,0753 \quad 17,2640 \quad 17,4890 \\
& P=\hat{m}_{k}-\hat{m}_{k} \text {, } \\
& \nabla(P)=\frac{2}{8} \cdot \mathrm{QM} \operatorname{Res} . \\
& \mathrm{DMS}=\mathrm{q} \cdot \sqrt{\frac{1}{2}\left(\frac{2}{8} \mathrm{QM} \operatorname{Res} .\right)}=1,4089 \\
& q_{0,05 ; 3,8}=4,04
\end{aligned}
$$


142 .

6. CONCLUSOES

6.1 - 0 modelo matemático proposto mostrou-se adequado quando se desejou testar a hipótese básica de nulidade sobre os tratamentos.

6.2 - O sistema de equações normais é semelhante ao ob tido por GODOI (1971). Quanto a parte que se refere aos efeitos de blocos, não encontramos na literatura dedução matemática que possibi litasse uma comparação con outros trabalhos.

6.3 - Somas de Quadrados

A expressão obtida para soma de quadrados de vida a efeitos de blocos coincide com aquela apresentada por LUCAS (1956). Para a soma de quadrados devida a efei- 
tos de tratamentos chegou-se ao mesmo resultado obtido por LUCAS (1956) e GODOI (1971). A fórmula obtida para a soma de quadrados devida a efeitos de vacas é a mesma proposta por GODOI (1971). Para a soma de quadrados devida a efeitos de períodos chegamos a resultados coinci dentes com as fórmulas apresentadas por BRANDT (1938) e GODOI (1971). A soma de quadrados devida a efeitos da interação período (linear) x vacas também coincide com o resultado apresentado por GODOI (1971).

6.4 - A análise de variância para ensaios como o aqui proposto mostrou-se eficiente quando se deseja testar a hipōtese da nulidade

$$
H_{0}: t_{1}=t_{2}=\cdots=t_{p}=0 \text {, }
$$

pois SQ Trat.(aj.) e SQR são independentes e seu quociente tem distribuição $F$.

Normalmente nesse tipo de ensaio não se tem interesse em testar os demais efeitos, o que seria possível visto às distribuições dos quocientes, exceto no caso de períodos onde o teste $F$ não é exato devidoà não independência das formas quadráticas de Período e Resíduo, obtidas através do modelo estudado. 
ANSCOMBE, F.J., 1961. Examination of Residuals. Fourth Berkely Symposium on Mathematical Statistics and Probability. Berkeley, 1(4): 1-36.

ANSCOMBE, F.J., 1973. Graphs in Statistical Analysis. American Statistician, 27: 17-21.

ANSCOMBE, F.J. e J.W. TUKEY, 1963. The Examination and Analysis of Residuals. Technometrics, $\underline{5}(2)$.

BRANDT, A.E., 1938. Tests of Significance in Reversal or Switchback Trials. Research Bulletin. Ames, 234: $60-87$.

CHAKRABARTI, M.C., 1962. Mathematics of Design and Ana1ysis of Experiments. 1a ed. Asia Publishing House, Londres, $120 \mathrm{pp}$. 
DRAPER, N.R. e H. SMITH, 1966. Applied Regression Analysis. Wiley, New York.

GILL, J.L., 1978. Design and Analysis of Experiments in the Animal and Medical Sciences. The Iowa State University Press. 1 a ed. Ames, 3 vol. 882 pp.

GODOI, C.R., 1971. Modelo Mátemático dos Ensaios em Dupla Reversão ("Switchback"). Escola Superior de Agricultura "Luiz de Queiroz"/USP, Piracicaba, 52 pp. [Teㅡ se de Doutoramento].

GODOI, C.R., 1983. Modelo linear com Auto-Correlação nos Resíduos para a Análise de Produção Leiteira em Experimentos com Dupla Reversão. Escola Superior de Agri cultura "Luiz de Queiroz"/USP, Piracicaba, 110 pp.[Te se de Livre Docência].

GRAYBILL, F.A., 1961. An Introduction to Linear Statis-. tical Models. Vol. I. McGraw-Hill.

IEMMA, A.F., 1981. Análise de Experimentos em Parcelas Subdivididas com Tratamentos Principais Dispostos em Blocos Incompletos Balanceados. Escola Superior de Agricultura "Luiz de Queiroz"/USP, Piracicaba, 145 pp. [Tese de Doutoramento].

KALIL, E.B., 1971. Princípios de Técnica Experimental com Animais. Instituto de Zootecnia de Nova Odessa, Nova Odessa. [Mimeografado]. 
LUCAS, H.L., 1951. Bias in Estimation of Error in Change-over Trials, with Dairy Cattle. Jour. Agric. Sci., 41: 146 .

LUCAS, H.L., 1956. Switchback Trials for More Than Two Treatments. Jour. Dairy Sci., 39: 146.

LUCAS, H.L., 1958. Designs in Animal Science Research Inst. of Stat. Univ. N.C. [Mimeografado].

NETER, J. e W. WASSERMAN, 1977 . Applied Linear Statistical Models. Regression, Analysis of Variance, and Experimental Designs. Richard D. Irwin, Inc., 1 a ed. I11inois, $841 \mathrm{pp}$.

PATTERSON, H.D., 1950. The analys is of Change-over Trials. Jour. Agric. Sci., 40: 375 .

PIMENTEL GOMES, F., 1967. The Solution of Normal Equations of Experiments in Incomplete Blocks. Ciência e Cultura. São Paulo, 20 : 733-746.

PIMENTEL GOMES, F., 1976. Curso de Estatistica Experimenta1. 6 a ed. Nobel, Piracicaba, $430 \mathrm{pp}$.

SCHEFFE, H., 1959. The Analysis of Variance. University of California, Berkeley, New York. John Wiley \& Sons, Inc., $477 \mathrm{pp}$.

SEARLE, S.R., 1971. Linear Mode1s. N.Y. State College of Agriculture, Cornell University. John Wiley \& Sons, Inc., $530 \mathrm{pp}$. 
SEBER, G.A.F., 1977. Linear Regression Analysis. Univer sity of Auckland, Auckland, New Zealand. John Wiley \& Sons, Inc., $466 \mathrm{pp}$.

TAYLOR, W.B. e P.J. ARMSTRONG, 1953. The Efficiency of Some Experimental Designs Used in Dairy Husbandry Experiments. J.Agr. Sci., 43: 407. 